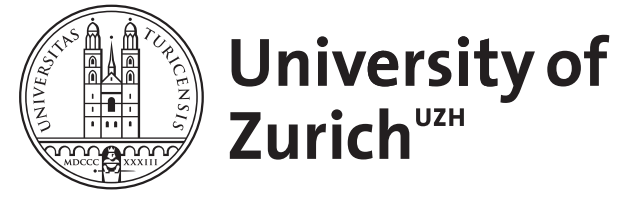

Zurich Open Repository and Archive

University of Zurich

University Library

Strickhofstrasse 39

CH-8057 Zurich

www.zora.uzh.ch

Year: 1999

\title{
Subordinators: examples and applications
}

Bertoin, Jean

DOI: https://doi.org/10.1007/978-3-540-48115-7_1

Posted at the Zurich Open Repository and Archive, University of Zurich

ZORA URL: https://doi.org/10.5167/uzh-79481

Book Section

Originally published at:

Bertoin, Jean (1999). Subordinators: examples and applications. In: Bernard, P. Lectures on Probability Theory and Statistics. Berlin: Springer, 1-91.

DOI: https://doi.org/10.1007/978-3-540-48115-7_1 


\section{SUBORDINATORS: EXAMPLES AND APPLICATIONS}

Ecole d'été de Probabilités de St-Flour, 1997

Jean BERTOIN

Laboratoire de Probabilités

Université Pierre et Marie Curie 


\section{Table of Contents}

0 Forewords 4

1 Elements on subordinators 5

1.1 Definitions and first properties . . . . . . . . . . . . . 5

1.2 The Lévy-Khintchine formula . . . . . . . . . . . . . . . . . . . 7

1.3 The renewal measure . . . . . . . . . . . . . . . . . . . . . . . . 9

1.4 The range of a subordinator . . . . . . . . . . . . . . . 13

$\begin{array}{lll}2 & \text { Regenerative property } & 17\end{array}$

2.1 Regenerative sets . . . . . . . . . . . . . . . . . 17

2.2 Connection with Markov processes . . . . . . . . . . . . . . . . . 19

3 Asymptotic behaviour of last passage times 23

3.1 Asymptotic behaviour in distribution . . . . . . . . . . . . . 23

3.1.1 The self-similar case . . . . . . . . . . . . . . . 23

3.1.2 The Dynkin-Lamperti theorem . . . . . . . . . . . . . 24

3.2 Asymptotic sample path behaviour . . . . . . . . . . . . . . . 27

4 Rates of growth of local time $\quad 31$

4.1 Law of the iterated logarithm . . . . . . . . . . . . . . 31

4.2 Modulus of continuity . . . . . . . . . . . . . . . 35

5 Geometric properties of regenerative sets 39

5.1 Fractal dimensions . . . . . . . . . . . . . . . . . . . . . . . . 39

5.1.1 Box-counting dimension . . . . . . . . . . . . . . . . . . . . . 39

5.1.2 Hausdorff and packing dimensions . . . . . . . . . . . . . . 41

5.2 Intersections with a regenerative set . . . . . . . . . . . . . 43

5.2.1 Equilibrium measure and capacity . . . . . . . . . . . . . . 43

5.2 .2 Dimension criteria . . . . . . . . . . . . . . 45

5.2 .3 Intersection of independent regenerative sets . . . . . . . . . . 47 
6 Burgers equation with Brownian initial velocity 50

6.1 Burgers equation and the Hopf-Cole solution . . . . . . . . . . . . 50

6.2 Brownian initial velocity . . . . . . . . . . . . . . . . . . 51

6.3 Proof of the theorem . . . . . . . . . . . . . . . . 52

7 Random covering $\quad 55$

7.1 Setting . . . . . . . . . . . . . . . . . 55

7.2 The Laplace exponent of the uncovered set . . . . . . . . . . . . . . 57

7.3 Some properties of the uncovered set . . . . . . . . . . . . . 58

8 Lévy processes $\quad 62$

8.1 Local time at a fixed point . . . . . . . . . . . . . . . . . . 62

8.2 Local time at the supremum . . . . . . . . . . . . . . . 65

8.3 The spectrally negative case . . . . . . . . . . . . . . . 67

8.4 Bochner's subordination for Lévy processes . . . . . . . . . . . . . . . . 69

9 Occupation times of a linear Brownian motion $\quad 73$

9.1 Occupation times and subordinators . . . . . . . . . . . 73

9.2 Lévy measure and Laplace exponent . . . . . . . . . . . . . . . . . . 74

9.2.1 Lévy measure via excursion theory . . . . . . . . . . . . . . 74

9.2.2 Laplace exponent via the Sturm-Liouville equation . . . . . . . 75

9.2.3 Spectral representation of the Laplace exponent . . . . . . . . . 77

9.3 The zero set of a one-dimensional diffusion . . . . . . . . . . . . . . . . 79 


\section{Forewords}

A subordinator is an increasing process that has independent and homogeneous increments. Subordinators thus form one of the simplest family of random processes in continuous time. The purpose of this course is two-fold: First to expose salient features of the theory and second to present a variety of examples and applications. The theory mostly concerns the statistical and sample path properties. The applications we have in mind essentially follow from the connection between subordinators and regenerative sets, that can be thought of as the set of times when a Markov process visits some fixed point of the state space. Typically, this enables us to translate certain problems on a given Markov process in terms of some subordinator, and then to use general known results on the latter.

Here is a sketch of the content. The first chapter introduces the basic notions and properties of subordinators, such as the Lévy-Khintchine formula, Itô's decomposition, renewal measures, ranges $\cdots$, and the second presents the correspondence relating subordinators, regenerative sets, and local times and excursions of Markov processes, which is essential to the future applications. More advanced material in that field is developed in chapters $3-5$, which concern respectively the asymptotic behaviour of last-passage times in connection with the Dynkin-Lamperti theorem, the smoothness of the local times (law of the iterated logarithm, modulus of continuity) and some geometric properties of regenerative sets including fractal dimensions and the study of the intersection with a given set. Applications are presented in chapters 6-9. First, we describe the law of the solution of the inviscid Burgers equation with Brownian initial velocity in terms of a subordinator, which enables us to investigate its statistical properties. Next, we study the closed subset of $[0, \infty)$ that is left uncovered by open intervals sampled from a Poisson point process, following the ingenious approach of Fitzsimmons et al. Then, we turn our attention to two natural regenerative sets associated with a real-valued Lévy process: The set of passage times at a fixed state, and the set of times when a new maximum is achieved. Some applications of Bochner's subordination for Lévy processes are also given. Finally we investigate the class of subordinators that appears in connection with occupation times of a linear Brownian motion, or, equivalently, with the zero set of one-dimensional diffusions, by making use of M. G. Krein's spectral theory of vibrating strings. The choice of the examples discussed here is quite arbitrary; for instance, Marsalle [117] exposes further applications in the same vein, to increase times of stable processes, slow or fast points for local times, and the favorite site of a Brownian motion with drift.

Last but not least, it is my pleasure to thank Marc Yor for his very valuable comments on the first draft of this work. 


\section{Chapter 1}

\section{Elements on subordinators}

The purpose of this chapter is to introduce basic notions on subordinators.

\subsection{Definitions and first properties}

Let $(\Omega, \mathbb{P})$ denote a probability space endowed with a right-continuous and complete filtration $\left(\mathcal{F}_{t}\right)_{t \geq 0}$. We consider right-continuous increasing adapted processes started from 0 and with values in the extended half-line $[0, \infty]$, where $\infty$ serves as a cemetery point (i.e. $\infty$ is an absorbing state). If $\sigma=\left(\sigma_{t}, t \geq 0\right)$ is such a process, we denote its lifetime by

$$
\zeta=\inf \left\{t \geq 0: \sigma_{t}=\infty\right\}
$$

and call $\sigma$ a subordinator if it has independent and homogeneous increments on $[0, \zeta)$. That is to say that for every $s, t \geq 0$, conditionally on $\{t<\zeta\}$, the increment $\sigma_{t+s}-\sigma_{t}$ is independent of $\mathcal{F}_{t}$ and has the same distribution as $\sigma_{s}$ (under $\mathbb{P}$ ). When the lifetime is infinite a.s., we say that $\sigma$ is a subordinator in the strict sense. The terminology has been introduced by Bochner [25]; see the forthcoming section 8.4.

Here is a standard example that will be further developed in Section 8.3. Consider a linear Brownian motion $B=\left(B_{t}: t \geq 0\right)$ started at 0 , and the first passage times

$$
\tau_{t}=\inf \left\{s \geq 0: B_{s}>t\right\}, \quad t \geq 0
$$

(it is well-known that $\tau_{t}<\infty$ for all $t \geq 0$, a.s.). We write $\mathcal{F}_{t}$ for the complete sigma-field generated by the Brownian motion stopped at time $\tau_{t}$, viz. $\left(B_{s \wedge \tau_{t}}: s \geq 0\right)$. According to the strong Markov property,

$$
B_{s}^{\prime}=B_{s+\tau_{t}}-t, \quad s \geq 0
$$

is independent of $\mathcal{F}_{t}$ and is again a Brownian motion. Moreover, it is clear that for every $s \geq 0$

$$
\tau_{t+s}-\tau_{t}=\inf \left\{u \geq 0: B_{u}^{\prime}>s\right\} .
$$

This shows that $\tau=\left(\tau_{t}: t \geq 0\right)$ is an increasing $\left(\mathcal{F}_{t}\right)$-adapted process with independent and homogeneous increments. Its paths are right-continuous and have an infinite lifetime a.s.; and hence $\tau$ is a strict subordinator. 
We assume henceforth that $\sigma$ is a subordinator. The independence and homogeneity of the increments immediately yield the (simple) Markov property: For every fixed $t \geq 0$, conditionally on $\{t<\zeta\}$, the process $\sigma^{\prime}=\left(\sigma_{s}^{\prime}=\sigma_{s+t}-\sigma_{t}, s \geq 0\right)$ is independent of $\mathcal{F}_{t}$ and has the same law as $\sigma$. The one-dimensional distributions of $\sigma$

$$
p_{t}(d y)=\mathbb{P}\left(\sigma_{t} \in d y, t<\zeta\right), \quad t \geq 0, y \in[0, \infty)
$$

thus give rise to a convolution semigroup $\left(P_{t}, t \geq 0\right)$ by

$$
P_{t} f(x)=\int_{[0, \infty)} f(x+y) p_{t}(d y)=\mathbb{E}\left(f\left(\sigma_{t}+x\right), t<\zeta\right)
$$

where $f$ stands for a generic nonnegative Borel function. It can be checked that this semigroup has the Feller property, cf. Proposition I.5 in [11] for details.

The simple Markov property can easily be reinforced, i.e. extended to stopping times:

Proposition 1.1 If $T$ is a stopping time, then, conditionally on $\{T<\zeta\}$, the process $\sigma^{\prime}=\left(\sigma_{t}^{\prime}=\sigma_{T+t}-\sigma_{T}, t \geq 0\right)$ is independent of $\mathcal{F}_{T}$ and has the same law as $\sigma$ (under $\mathbb{P})$.

Proof: For an elementary stopping time, the statement merely rephrases the simple Markov property. If $T$ is a general stopping time, then there exists a sequence of elementary stopping times $\left(T_{n}\right)_{n \in \mathbb{N}}$ that decrease towards $T$, a.s. For each integer $n$, conditionally on $\left\{T_{n}<\zeta\right\}$, the shifted process $\left(\sigma_{T_{n}+t}-\sigma_{T_{n}}, t \geq 0\right)$ is independent of $\mathcal{F}_{T_{n}}$ (and thus of $\mathcal{F}_{T}$ ), and has the same law as $\sigma$. Letting $n \rightarrow \infty$ and using the right-continuity of the paths, this entails our assertion.

The law of a subordinator is specified by the Laplace transforms of its onedimensional distributions. To this end, it is convenient to use the convention that $\mathrm{e}^{-\lambda \times \infty}=0$ for any $\lambda \geq 0$, so that

$$
\mathbb{E}\left(\exp \left\{-\lambda \sigma_{t}\right\}, t<\zeta\right)=\mathbb{E}\left(\exp \left\{-\lambda \sigma_{t}\right\}\right), \quad t, \lambda \geq 0 .
$$

The independence and homogeneity of the increments then yield the multiplicative property

$$
\mathbb{E}\left(\exp \left\{-\lambda \sigma_{t+s}\right\}\right)=\mathbb{E}\left(\exp \left\{-\lambda \sigma_{t}\right\}\right) \mathbb{E}\left(\exp \left\{-\lambda \sigma_{s}\right\}\right)
$$

for every $s, t \geq 0$. We can therefore express these Laplace transforms in the form

$$
\mathbb{E}\left(\exp \left\{-\lambda \sigma_{t}\right\}\right)=\exp \{-t \Phi(\lambda)\}, \quad t, \lambda \geq 0
$$

where the function $\Phi:[0, \infty) \rightarrow[0, \infty)$ is called the Laplace exponent of $\sigma$.

Returning to the example of the first passage process $\tau$ of a linear Brownian motion, one can use the scaling property of Brownian motion and the reflexion principle to determine the distribution of $\tau_{1}$. Specifically, for every $t>0$

$$
\begin{aligned}
\mathbb{P}\left(\tau_{1}<t\right)=\mathbb{P}\left(\sup _{0 \leq s \leq t} B_{s}>1\right)=\mathbb{P}\left(\sup _{0 \leq s \leq 1} B_{s}>1 / \sqrt{t}\right) & =\mathbb{P}\left(\left|B_{1}\right|>1 / \sqrt{t}\right) \\
& =\sqrt{\frac{2}{\pi}} \int_{0}^{t} s^{-3 / 2} \mathrm{e}^{-1 / 2 s} d s
\end{aligned}
$$


It is easy to deduce that the Laplace exponent of $\tau$ is

$$
\Phi(\lambda)=-\log \mathbb{E}\left(\exp \left\{-\lambda \tau_{1}\right\}\right)=\sqrt{2 \lambda} .
$$

\subsection{The Lévy-Khintchine formula}

The next theorem gives a necessary and sufficient analytic condition for a function to be the Laplace exponent of a subordinator.

Theorem 1.2 (de Finetti, Lévy, Khintchine)(i) If $\Phi$ is the Laplace exponent of a subordinator, then there exist a unique pair $(\mathrm{k}, \mathrm{d})$ of nonnegative real numbers and a unique measure $\Pi$ on $(0, \infty)$ with $\int(1 \wedge x) \Pi(d x)<\infty$, such that for every $\lambda \geq 0$

$$
\Phi(\lambda)=\mathrm{k}+\mathrm{d} \lambda+\int_{(0, \infty)}\left(1-\mathrm{e}^{-\lambda x}\right) \Pi(d x) .
$$

(ii) Conversely, any function $\Phi$ that can be expressed in the form (1.2) is the Laplace exponent of a subordinator.

Equation (1.2) will be referred to as the Lévy-Khintchine formula; one calls $\mathrm{k}$ the killing rate, $\mathrm{d}$ the drift coefficient and $\Pi$ the Lévy measure of $\sigma$. It is sometimes convenient to perform an integration by parts and rewrite the Lévy-Khintchine formula as

$$
\Phi(\lambda) / \lambda=\mathrm{d}+\int_{0}^{\infty} \mathrm{e}^{-\lambda x} \bar{\Pi}(x) d x, \quad \text { with } \bar{\Pi}(x)=\mathrm{k}+\Pi((x, \infty)) .
$$

We call $\bar{\Pi}$ the tail of the Lévy measure. Note that the killing rate and the drift coefficient are given by

$$
\mathrm{k}=\Phi(0) \quad, \quad \mathrm{d}=\lim _{\lambda \rightarrow \infty} \frac{\Phi(\lambda)}{\lambda}
$$

In particular, the lifetime $\zeta$ has an exponential distribution with parameter $\mathrm{k} \geq 0$ $(\zeta \equiv \infty$ for $\mathrm{k}=0)$.

Before we proceed to the proof of Theorem 1.2, we present some well-known examples of subordinators. The simplest is the Poisson process with intensity $c>0$, which corresponds to the Laplace exponent

$$
\Phi(\lambda)=c\left(1-\mathrm{e}^{-\lambda}\right)
$$

that is the killing rate $\mathrm{k}$ and the drift coefficient $\mathrm{d}$ are zero and the Lévy measure $c \delta_{1}$, where $\delta_{1}$ stands for the Dirac point mass at 1 . Then the so-called standard stable subordinator with index $\alpha \in(0,1)$ has a Laplace exponent given by

$$
\Phi(\lambda)=\lambda^{\alpha}=\frac{\alpha}{\Gamma(1-\alpha)} \int_{0}^{\infty}\left(1-\mathrm{e}^{-\lambda x}\right) x^{-1-\alpha} d x .
$$

The restriction on the range of the index is due to the requirement $\int(1 \wedge x) \Pi(d x)<$ $\infty$. The boundary case $\alpha=1$ is degenerate since it corresponds to the deterministic process $\sigma_{t} \equiv t$, and is usually implicitly excluded. A third family of examples is 
provided by the Gamma processes with parameters $a, b>0$, for which the Laplace exponent is

$$
\Phi(\lambda)=a \log (1+\lambda / b)=\int_{0}^{\infty}\left(1-\mathrm{e}^{-\lambda x}\right) a x^{-1} \mathrm{e}^{-b x} d x,
$$

where the second equality stems from the Frullani integral. We see that the Lévy measure is $\Pi^{(a, b)}(d x)=a x^{-1} \mathrm{e}^{-b x} d x$ and the killing rate and the drift coefficient are zero.

Proof of Theorem 1.2: (i) Making use of the independence and homogeneity of the increments in the second equality below, we get from (1.1) that for every $\lambda \geq 0$

$$
\begin{aligned}
\Phi(\lambda)=\lim _{n \rightarrow \infty} n(1-\exp \{-\Phi(\lambda) / n\}) & =\lim _{n \rightarrow \infty} n \mathbb{E}\left(1-\exp \left\{-\lambda \sigma_{1 / n}\right\}\right) \\
& =\lambda \lim _{n \rightarrow \infty} \int_{0}^{\infty} \mathrm{e}^{-\lambda x} n \mathbb{P}\left(\sigma_{1 / n} \geq x\right) d x .
\end{aligned}
$$

Write $\bar{\Pi}_{n}(x)=n \mathbb{P}\left(\sigma_{1 / n} \geq x\right)$, so that

$$
\frac{\Phi(\lambda)}{\lambda}=\lim _{n \rightarrow \infty} \int_{0}^{\infty} \mathrm{e}^{-\lambda x} \bar{\Pi}_{n}(x) d x .
$$

This shows that the sequence of absolutely continuous measures $\bar{\Pi}_{n}(x) d x$ converges vaguely as $n \rightarrow \infty$. As each function $\bar{\Pi}_{n}(\cdot)$ decreases, the limit has necessarily the form $\mathrm{d} \delta_{0}(d x)+\bar{\Pi}(x) d x$, where $\mathrm{d} \geq 0, \bar{\Pi}:(0, \infty) \rightarrow[0, \infty)$ is a non-increasing function, and $\delta_{0}$ stands for the Dirac point mass at 0 . Thus

$$
\frac{\Phi(\lambda)}{\lambda}=\mathrm{d}+\int_{0}^{\infty} \mathrm{e}^{-\lambda x} \bar{\Pi}(x) d x
$$

and this yields (1.2) with $\mathrm{k}=\bar{\Pi}(\infty)$ and $\Pi(d x)=-d \bar{\Pi}(x)$ on $(0, \infty)$. It is plain that we must have $\int_{(0,1)} x \Pi(d x)<\infty$ since otherwise $\Phi(\lambda)$ would be infinite. Uniqueness is obvious.

(ii) Consider a Poisson point process $\Delta=\left(\Delta_{t}, t \geq 0\right)$ with values in $(0, \infty]$ and with characteristic measure $\Pi+\mathrm{k} \delta_{\infty}$. This means that for every Borel set $B \subseteq(0, \infty]$, the counting process $N^{B}=\operatorname{Card}\left\{s \in[0, \cdot]: \Delta_{s} \in B\right\}$ is a Poisson process with intensity $\Pi(B)+\mathrm{k} \delta_{\infty}(B)$, and to disjoint Borel sets correspond independent Poisson processes. In particular, the instant of the first infinite point, $\tau_{\infty}=\inf \left\{t \geq 0: \Delta_{t}=\infty\right\}$, has an exponential distribution with parameter $\mathrm{k}\left(\tau_{\infty} \equiv \infty\right.$ if $\left.\mathrm{k}=0\right)$, and is independent of the Poisson point process restricted to $(0, \infty)$. Moreover, the latter is a Poisson point process with characteristic measure $\Pi$.

Introduce $\Sigma=\left(\Sigma_{t}, t \geq 0\right)$ by

$$
\Sigma_{t}=\mathrm{d} t+\sum_{0 \leq s \leq t} \Delta_{s}
$$

The condition $\int(1 \wedge x) \Pi(d x)<\infty$ ensures that $\Sigma_{t}<\infty$ whenever $t<\tau_{\infty}$, a.s. It is plain that $\Sigma$ is a right-continuous increasing process started at 0 , with lifetime $\tau_{\infty}$, and that its increments are stationary and independent on $\left[0, \tau_{\infty}\right)$. In other words, $\Sigma$ is a subordinator. Finally, the exponential formula for a Poisson point process (e.g. Proposition XII.1.12 in [132]) gives for every $t, \lambda \geq 0$

$$
\mathbb{E}\left(\exp \left\{-\lambda \Sigma_{t}\right\}\right)=\exp \left\{-t\left(\mathrm{k}+\mathrm{d} \lambda+\int_{(0, \infty)}\left(1-\mathrm{e}^{-\lambda x}\right) \Pi(d x)\right)\right\}
$$


which shows that the Laplace exponent of $\Sigma$ is given by (1.2).

More precisely, the proof of (ii) contains relevant information on the canonical decomposition of a subordinator as the sum of its continuous part and its jumps.

Proposition 1.3 (Itô [81]) One has a.s., for every $t \geq 0$ :

$$
\sigma_{t}=\mathrm{d} t+\sum_{0 \leq s \leq t} \Delta_{s}
$$

where $\Delta=\left(\Delta_{s}, s \geq 0\right)$ is a Poisson point process with values in $(0, \infty]$ and characteristic measure $\Pi+\mathrm{k} \delta_{\infty}$, where $\delta_{\infty}$ stands for the Dirac point mass at $\infty$. The lifetime of $\sigma$ is then given by $\zeta=\inf \left\{t \geq 0: \Delta_{t}=\infty\right\}$.

As a consequence, we see that a subordinator is a step process if its drift coefficient is $\mathrm{d}=0$ and its Lévy measure has a finite mass, $\Pi((0, \infty))<\infty$ (this is also equivalent to the boundedness of the Laplace exponent). Otherwise $\sigma$ is a strictly increasing process. In the first case, we say that $\sigma$ is a compound Poisson process. A compound Poisson process can be identified as a random walk time-changed by an independent Poisson process; and in many aspects, it can be thought of as a process in discrete time. Because we are mostly concerned with 'truly' continuous time problems, it will be more convenient to concentrate on strictly increasing subordinators in the sequel.

Henceforth, the case when $\sigma$ is a compound Poisson process is implicitly excluded. ${ }^{1}$

\subsection{The renewal measure}

A subordinator is a transient Markov process; its potential measure $U(d x)$ is called the renewal measure. It is given by

$$
\int_{[0, \infty)} f(x) U(d x)=\mathbb{E}\left(\int_{0}^{\infty} f\left(\sigma_{t}\right) d t\right) .
$$

The distribution function of the renewal measure

$$
U(x)=\mathbb{E}\left(\int_{0}^{\infty} \mathbf{1}_{\left\{\sigma_{t} \leq x\right\}} d t\right), \quad x \geq 0
$$

is known as the renewal function. If we introduce the continuous inverse of the strictly increasing process $\sigma$ :

$$
L_{x}=\sup \left\{t \geq 0: \sigma_{t} \leq x\right\}=\inf \left\{t>0: \sigma_{t}>x\right\}, \quad x \geq 0,
$$

we then see that

$$
U(x)=\mathbb{E}\left(L_{x}\right) ;
$$

\footnotetext{
${ }^{1}$ Nonetheless, many results presented in this text still hold in the general case.
} 
in particular we obtain by an application of the theorem of dominated convergence that the renewal function is continuous. It is also immediate to deduce from the Markov property that the renewal function is subadditive, that is

$$
U(x+y) \leq U(x)+U(y) \quad \text { for all } x, y \geq 0 .
$$

Because the Laplace transform of the renewal measure is

$$
\mathcal{L} U(\lambda)=\int_{[0, \infty)} \mathrm{e}^{-\lambda x} U(d x)=\frac{1}{\Phi(\lambda)}, \quad \lambda>0
$$

the renewal measure characterizes the law of the subordinator.

We next present useful estimations for the renewal measure in terms of the Laplace exponent and of the tail of the Lévy measure, which follow from the fact that the Laplace transforms of $U$ and $\bar{\Pi}$ admit simple expressions in terms of $\Phi$, and adequate Tauberian theorems. To this end, we first state a general result. When $f$ and $g$ are two nonnegative functions, we use the notation $f \asymp g$ to indicate that there are two positive constants, $c$ and $c^{\prime}$, such that $c f \leq g \leq c^{\prime} f$. Introduce the so-called integrated tail

$$
I(t)=\int_{0}^{t} \bar{\Pi}(x) d x=\int_{0}^{t}(\mathrm{k}+\Pi((x, \infty))) d x .
$$

Proposition 1.4 We have

$$
U(x) \asymp 1 / \Phi(1 / x) \text { and } \Phi(x) / x \asymp I(1 / x)+\mathrm{d} .
$$

Proof: Recall that $1 / \Phi$ is the Laplace transform of the renewal measure. As $\Phi$ is concave and monotone increasing, the Tauberian theorem of de Haan and Stadtmüller (see [20] on page 118) applies and yields $U(x) \asymp 1 / \Phi(1 / x)$. The second estimate follows similarly, using the fact that the Laplace transform of the tail of the Lévy measure is $-\mathrm{d}+\Phi(\lambda) / \lambda$ (by the Lévy-Khintchine formula).

Sharper estimates follow from Karamata's Tauberian theorem when one imposes that the Laplace exponent has regular variation. Recall that a measurable function $f:(0, \infty) \rightarrow[0, \infty)$ is regularly varying at $0+$ (respectively, at $\infty)$ if for every $x>0$, the ratio $f(\lambda x) / f(\lambda)$ converges as $\lambda \rightarrow 0+$ (respectively, $\lambda \rightarrow \infty$ ). The limit is then necessarily $x^{\alpha}$ for some real number $\alpha$ which is called the index. When $\alpha=0$, we will simply say that $f$ is slowly varying. We refer to Chapter XIII in Feller [53] for the basic theory, and to Bingham et al. [20] for the complete account. We stress that when the Laplace exponent $\Phi$ is regularly varying (at $0+$ or at $\infty$ ) then, due to the Lévy-Khintchine formula, the index necessarily lies between 0 and 1 .

Proposition 1.5 Suppose that $\Phi$ is regularly varying at $0+$ (respectively, at $\infty$ ) with index $\alpha \in[0,1]$. Then,

$$
\Gamma(1+\alpha) U(a x) \sim a^{\alpha} / \Phi(1 / x) \quad \text { as } x \rightarrow \infty \text { (respectively, as } x \rightarrow 0+\text { ), }
$$

uniformly as a varies on any fixed compact interval of $(0, \infty)$.

Moreover, if $\alpha<1$, then

$$
\left.\Gamma(1-\alpha) \bar{\Pi}(a x) \sim a^{-\alpha} \Phi(1 / x) \quad \text { as } x \rightarrow \infty \text { (respectively, as } x \rightarrow 0+\right),
$$

uniformly as a varies on any fixed compact interval of $(0, \infty)$ 
Proof: The first assertion follows from Karamata's Tauberian theorem and the uniform convergence theorem; cf. Theorems 1.7.1 and 1.5.2 in [20]. The second requires the monotone density theorem; see Theorem 1.7.2 in [20].

Next, local estimates for the renewal measure in the neighbourhood of $\infty$ are given by the renewal theorem.

Proposition 1.6 (Renewal theorem) Put $\mathbb{E}\left(\sigma_{1}\right)=\mu \in(0, \infty]$. Then for every $h>0$

$$
\lim _{x \rightarrow \infty}(U(x+h)-U(x))=h / \mu .
$$

This renewal theorem is essentially a consequence of the standard renewal theorem in discrete time (i.e. for so-called renewal processes; see e.g. Feller [53]). Recall that the compound Poisson case has been ruled out, so $\sigma$ is a 'non-lattice' process. Plainly, it is mostly useful in the finite mean case $\mu<\infty$; we refer to Doney [48] for recent progress in the (discrete) infinite mean case.

There is also an analogue of the renewal theorem in the neighbourhood of $0+$ when the drift coefficient is positive.

Proposition 1.7 (Neveu [122]) Suppose that $\mathrm{d}>0$. Then the renewal measure is absolutely continuous and has a continuous everywhere positive density $u:[0, \infty) \rightarrow$ $(0, \infty)$ given by

$$
u(x)=\mathrm{d}^{-1} \mathbb{P}\left(\exists t \geq 0: \sigma_{t}=x\right) .
$$

In particular, $u(0)=1 / \mathrm{d}$.

Proof: As $d>0$, the Laplace transform of the renewal measure has

$$
\int_{0}^{\infty} \mathrm{e}^{-\lambda x} U(d x)=\frac{1}{\Phi(\lambda)} \sim \frac{1}{\mathrm{~d} \lambda} \quad \text { as } \lambda \rightarrow \infty .
$$

By a Tauberian theorem, this entails

$$
U(\varepsilon) \sim \varepsilon / \mathrm{d}=\varepsilon u(0) \quad \text { as } \varepsilon \rightarrow 0+.
$$

The Markov property applied at the stopping time $L(x)=\inf \left\{t \geq 0: \sigma_{t}>x\right\}$ gives

$$
\begin{aligned}
U(x+\varepsilon)-U(x) & =\mathbb{E}\left(\int_{L(x)}^{\infty} \mathbf{1}_{\left\{\sigma_{t} \in(x, x+\varepsilon]\right\}} d t\right) \\
& =\int_{[x, x+\varepsilon]} \mathbb{P}\left(\sigma_{L(x)} \in d y\right) U(x+\varepsilon-y) \\
& =\mathbb{P}\left(\sigma_{L(x)}=x\right) U(\varepsilon)+\int_{(x, x+\varepsilon]} \mathbb{P}\left(\sigma_{L(x)} \in d y\right) U(x+\varepsilon-y) .
\end{aligned}
$$

The second term in the sum is bounded from above by $\mathbb{P}\left(\sigma_{L(x)} \in(x, x+\varepsilon]\right) U(\varepsilon)=$ $o(U(\varepsilon))$. We deduce from (1.3) that

$$
\mathrm{d}^{-1} \mathbb{P}\left(\exists t: \sigma_{t}=x\right)=\mathrm{d}^{-1} \mathbb{P}\left(\sigma_{L(x)}=x\right)=\lim _{\varepsilon \rightarrow 0+} \frac{U(x+\varepsilon)-U(x)}{\varepsilon}
$$


(the first equality stems from the fact that $L(x)$ depends continuously on $x$ ). In particular, the renewal measure is absolutely continuous; we henceforth denote by $u(x)$ the version of its density that is specified by the last displayed formula. Note that $u(x) \leq 1 / \mathrm{d}$ and also, by an immediate application of the Markov property at $L(x)$, that for every $x, y \geq 0$

$$
\mathrm{d} u(x+y)=\mathbb{P}\left(\exists t: \sigma_{t}=x+y\right) \geq \mathbb{P}\left(\exists t: \sigma_{t}=x\right) \mathbb{P}\left(\exists t: \sigma_{t}=y\right)=\mathrm{d}^{2} u(x) u(y) .
$$

To prove the continuity of $u$ at $x=0$, fix $\eta>0$ and consider the Borel set $B_{\eta}=\{x \geq 0: 1 / \mathrm{d} \leq u(x)+\eta\}$. As $u$ is bounded from above by $1 / \mathrm{d}$, we see from (1.3) that 0 is a point of density of $B_{\eta}$, in the sense that $m\left([0, \varepsilon] \cap B_{\eta}\right) \sim \varepsilon$ as $\varepsilon \rightarrow 0+$, where $m$ stands for the Lebesgue measure. By a standard result of measure theory, this implies that for some $a>0$ and every $0<x<a$, we can find $y, y^{\prime} \in B_{\eta}$ such that $x=y+y^{\prime}$. Using (1.4), we deduce

$$
u(x) \geq \mathrm{d} u(y) u\left(y^{\prime}\right) \geq \mathrm{d}\left(\frac{1}{\mathrm{~d}}-\eta\right)^{2},
$$

so that $\lim _{x \rightarrow 0+} u(x)=1 / \mathrm{d}=u(0)$.

We next prove the continuity at some arbitrary $x>0$. The same argument as above based on (1.4) yields

$$
\limsup _{y \rightarrow x-} u(y) \leq u(x) \leq \liminf _{y \rightarrow x+} u(y) .
$$

On the one hand, the right-continuity of the paths shows that if $y_{n}$ is a sequence that decreases towards $x$, then

$$
\limsup \left\{\exists t: \sigma_{t}=y_{n}\right\} \subseteq\left\{\exists t: \sigma_{t}=x\right\},
$$

so an application of Fatou's lemma gives

$$
\limsup _{y \rightarrow x+} u(y) \leq u(x) .
$$

On the other hand, an application of the Markov property as in (1.4) yields that for every $\varepsilon>0$

$$
\mathbb{P}\left(\exists t: \sigma_{t}=x\right) \leq \mathbb{P}\left(\exists t: \sigma_{t}=\varepsilon\right) \mathbb{P}\left(\exists t: \sigma_{t}=x-\varepsilon\right)+\mathbb{P}\left(\forall t: \sigma_{t} \neq \varepsilon\right) .
$$

We know that the second term in the sum tends to 0 as $\varepsilon \rightarrow 0+$, so that

$$
\liminf _{y \rightarrow x-} u(y) \geq u(x),
$$

and the continuity of $u$ is proven. Finally, we know that $u$ is positive in some neighbourhood of 0 , and it follows from (1.4) that $u$ is positive everywhere.

To conclude this section, we mention that large deviations estimates for the onedimensional distributions of $\sigma$ have been obtained by Jain and Pruitt [87]; see also Fristedt and Pruitt [61] for some more elementary results in that field. 


\subsection{The range of a subordinator}

The range of a subordinator $\sigma$ is the random closed subset of $[0, \infty)$ defined by

$$
\mathcal{R}=\overline{\left\{\sigma_{t}: 0 \leq t<\zeta\right\}} .
$$

Note that $\mathcal{R}$ is a perfect (i.e. without isolated points) and $0 \in \mathcal{R}$. Because the paths of $\sigma$ are càdlàg, the range can also be expressed as

$$
\mathcal{R}=\left\{\sigma_{t}: 0 \leq t<\zeta\right\} \bigcup\left\{\sigma_{s-}: s \in \mathcal{J}\right\}
$$

where $\mathcal{J}=\left\{0 \leq s \leq \zeta: \Delta_{s}>0\right\}$ denotes the set of jump times of $\sigma$. To this end, observe that $\left\{\sigma_{s-}: s \in \mathcal{J}\right\}$ is precisely the set of points in $\mathcal{R}$ that are isolated on their right. Alternatively, the canonical decomposition of the open set $\mathcal{R}^{c}=[0, \infty)-\mathcal{R}$, is

$$
\mathcal{R}^{c}=\bigcup_{s \in \mathcal{J}}\left(\sigma_{s-}, \sigma_{s}\right) .
$$

Recall that $L .=\inf \left\{t \geq 0: \sigma_{t}>\cdot\right\}$ stands for the -continuous- inverse of $\sigma$; it should be plain that $\mathcal{R}$ also coincides with the support of the Stieltjes measure $d L$ :

$$
\mathcal{R}=\operatorname{Supp}(d L),
$$

which provides another useful representation of the range.

We next present basic properties of the range that will be useful in the sequel. First, an interesting problem that frequently arises about random sets, is the evaluation of their sizes. The simplest result in that field for the range of a subordinator concerns its Lebesgue measure. Sharper results involving Hausdorff and packing dimension will be presented in section 5.1.

Proposition 1.8 We have

$$
m(\mathcal{R} \cap[0, t])=m\left(\left\{\sigma_{s}: s \geq 0\right\} \cap[0, t]\right)=\mathrm{d} L_{t} \quad \text { a.s. for all } t \geq 0,
$$

where $\mathrm{d}$ is the drift coefficient and $m$ the Lebesgue measure on $[0, \infty)$. In particular $\mathcal{R}$ has zero Lebesgue measure a.s. if and only if $\mathrm{d}=0$, and we then say that $\mathcal{R}$ is light. Otherwise we say that $\mathcal{R}$ is heavy.

Proof: The first equality is obvious as $\mathcal{R}$ differs from $\left\{\sigma_{s}: s \geq 0\right\}$ by at most countably many points. Next note that it suffices to treat the case $\mathrm{k}=0$ (i.e. $\zeta=\infty$ a.s.), because the case $\mathrm{k}>0$ then will then follow by introducing a killing at some independent time.

Recall that the canonical decomposition of the complementary set $\mathcal{R}^{c}$ is given by (1.5). In particular, for every fixed $t \geq 0$, the Lebesgue measure of $\mathcal{R}^{c} \cap\left[0, \sigma_{t}\right]$ is $\sum_{s \leq t} \Delta_{s}$, and the latter quantity equals $\sigma_{t}-\mathrm{d} t$ by virtue of Proposition 1.3. This gives $m\left(\left[0, \sigma_{t}\right] \cap \mathcal{R}\right)=\mathrm{d} t$ for all $t \geq 0$, a.s. Because the quantity on the right depends continuously on $t$, this entails by an argument of monotonicity that

$$
m\left(\left[0, \sigma_{t}\right] \cap \mathcal{R}\right)=m\left(\left[0, \sigma_{t-}\right] \cap \mathcal{R}\right)=\mathrm{d} t .
$$

Replacing $t$ by $L_{t}$ and recalling that $t \in\left[\sigma_{L_{t}-}, \sigma_{L_{t}}\right]$ completes the proof.

We then specify the probability that $x \in \mathcal{R}$ for any fixed $x>0$. 
Proposition 1.9 (i) (Kesten [96]) If the drift is $\mathrm{d}=0$, then $\mathbb{P}(x \in \mathcal{R})=0$ for every $x>0$.

(ii) (Neveu [122]) If $\mathrm{d}>0$, then the function $u(x)=\mathrm{d}^{-1} \mathbb{P}(x \in \mathcal{R})$ is the version of the renewal density $d U(x) / d x$ that is continuous and everywhere positive on $[0, \infty)$.

Proof: (i) An application of Fubini's theorem gives

$$
\int_{0}^{\infty} \mathbb{P}(x \in \mathcal{R}) d x=\mathbb{E}(m(\mathcal{R}))
$$

where $m(\mathcal{R})$ stands for the Lebesgue measure of $\mathcal{R}$. We know from Proposition 1.8 that the latter is zero as $\mathrm{d}=0$. In other words, $\mathbb{P}(x \in \mathcal{R})=0$ for almost every $x \geq 0$. That we may drop "almost" in the last sentence is easily seen when the renewal measure is absolutely continuous. More precisely, let $\tau$ be an independent random time with an exponential distribution with parameter 1 . For every fixed $q>0$, we have for any Borel set $A$

$$
\mathbb{P}\left(\sigma_{\tau / q} \in A\right)=q \int_{0}^{\infty} \mathrm{e}^{-q t} \mathbb{P}\left(\sigma_{t} \in A\right) d t \leq q U(A)
$$

which implies by virtue of the Radon-Nikodym theorem that the distribution of $\sigma_{\tau / q}$ is also absolutely continuous. Applying the Markov property at time $\tau / q$, we deduce that

$$
\mathbb{P}\left(\sigma_{\tau / q+t}=x \text { or } \sigma_{\tau / q+t-}=x \text { for some } t>0\right)=\int_{0}^{\infty} \mathbb{P}\left(\sigma_{\tau / q} \in d y\right) \mathbb{P}(x-y \in \mathcal{R}),
$$

and the right-hand side equals zero as $\mathbb{P}(a \in \mathcal{R})=0$ for almost every $a>0$. Letting $q$ go to $\infty$, we deduce that $\mathbb{P}(x \in \mathcal{R})=0$ for every $x>0$.

The same holds true even when the renewal measure is not absolutely continuous. This requires a more delicate analysis; we refer to the proof of Theorem III.4 in [11] for details.

(ii) By Proposition 1.7, all that is needed is to check that

$$
\mathbb{P}\left(\exists t>0: \sigma_{t-}=x<\sigma_{t}\right)=0 .
$$

By the compensation formula for Poisson point processes, we have for every $\varepsilon>0$

$$
\begin{aligned}
\mathbb{P}\left(\exists t>0: \sigma_{t-}=x<\sigma_{t}-\varepsilon\right) & =\mathbb{E}\left(\sum_{t \geq 0} \mathbf{1}_{\left\{\sigma_{t-}=x\right\}} \mathbf{1}_{\left\{\Delta_{t}>\varepsilon\right\}}\right) \\
& =\bar{\Pi}(\varepsilon) \mathbb{E}\left(\int_{0}^{\infty} \mathbf{1}_{\left\{\sigma_{t}=x\right\}} d t\right)
\end{aligned}
$$

and the ultimate quantity is zero as the renewal measure has no atom. As $\varepsilon$ is arbitrary, this completes the proof.

We next turn our attention to the left and right extremities of $\mathcal{R}$ as viewed from a fixed point $t \geq 0$ :

$$
g_{t}=\sup \{s<t: s \in \mathcal{R}\} \quad \text { and } \quad D_{t}=\inf \{s>t: s \in \mathcal{R}\} \text {. }
$$


We call $\left(D_{t}: t \geq 0\right)$ and $\left(g_{t}: t>0\right)$ the processes of first-passage and last-passage in $\mathcal{R}$, respectively. The use of an upper-case letter (respectively, of a lower-case letter) refers to the right-continuity (respectively, the left-continuity) of the sample paths. We immediately check that these processes can be expressed in terms of $\sigma$ and its inverse $L$ as follows :

$$
g_{t}=\sigma\left(L_{t}-\right) \quad \text { and } \quad D_{t}=\sigma\left(L_{t}\right) \quad \text { for all } t \geq 0, \text { a.s. }
$$

We present an useful expression for the distribution of the pair $\left(g_{t}, D_{t}\right)$ in terms of the renewal function and the tail of the Lévy measure.

Lemma 1.10 For every real numbers $a, b, t$ such that $0 \leq a<t \leq a+b$, we have

$$
\mathbb{P}\left(g_{t} \in d a, D_{t}-g_{t} \in d b\right)=\Pi(d b) U(d a) \quad, \quad \mathbb{P}\left(g_{t} \in d a, D_{t}=\infty\right)=\mathrm{k} U(d a) .
$$

In particular, we have for $a \in[0, t)$

$$
\mathbb{P}\left(g_{t} \in d a\right)=\bar{\Pi}(t-a) U(d a) .
$$

Proof: Recall from (1.6) the identities $g_{t}=\sigma_{L_{t}-}$ and $D_{t}-g_{t}=\Delta_{L_{t}}$. Then observe that for any $u>0$

$$
\sigma_{L_{t-}}<a \text { and } L_{t}=u \Longleftrightarrow \sigma_{u-}<a \text { and } \sigma_{u} \geq t
$$

Using the canonical expression of $\sigma$ given in Proposition 1.3, we see that

$$
\mathbb{P}\left(g_{t}<a, D_{t}-g_{t} \geq b\right)=\mathbb{E}\left(\sum \mathbf{1}_{\left\{\sigma_{u-}<a\right\}} \mathbf{1}_{\left\{\Delta_{u} \geq\left(t-\sigma_{u-}\right) \vee b\right\}}\right),
$$

where the sum in the right-hand side is taken over all the instants when the point process $\Delta$ jumps. The process $u \rightarrow \sigma_{u-}$ is left continuous and hence predictable, so the compensation formula (see e.g. Proposition XII.1.10 in [132]) entails that the right-hand-side in the last displayed formula equals

$$
\mathbb{E}\left(\int_{0}^{\infty} \mathbf{1}_{\left\{\sigma_{u}<a\right\}} \bar{\Pi}\left(\left(\left(t-\sigma_{u}\right) \vee b\right)-\right) d u\right)=\int_{[0, a)} \bar{\Pi}(((t-x) \vee b)-) U(d x) .
$$

This shows that for $0 \leq a<t<a+b$

$$
\mathbb{P}\left(g_{t} \in d a, D_{t}-g_{t} \in d b\right)=\Pi(d b) U(d a) .
$$

Integrating this when $b$ ranges over $[t-a, \infty]$ yields $\mathbb{P}\left(g_{t} \in d a\right)=\bar{\Pi}((t-a)-) U(d a)$. Since the renewal measure has no atom and the tail of the Lévy measure has at most countably many discontinuities, we may replace $\bar{\Pi}((t-a)-)$ by $\bar{\Pi}(t-a)$.

A possible drawback of Lemma 1.10 is that it is not expressed explicitly in terms of the Laplace exponent $\Phi$. Considering a double Laplace transform easily yields the following formula.

Lemma 1.11 For every $\lambda, q>0$

$$
\int_{0}^{\infty} \mathrm{e}^{-q t} \mathbb{E}\left(\exp \left\{-\lambda g_{t}\right\}\right) d t=\frac{\Phi(q)}{q \Phi(\lambda+q)} .
$$


Proof: It is immediately seen from Lemma 1.10 that $\mathbb{P}\left(g_{t}<t=D_{t}\right)=0$ for every $t>0$; it follows that $\mathbb{P}\left(g_{t}=t\right)=\mathbb{P}(t \in \mathcal{R})$. Using Proposition 1.9 and the fact that the Laplace transform of the renewal measure is $1 / \Phi$, we find

$$
\int_{0}^{\infty} \mathrm{e}^{-q t} \mathbb{P}\left(g_{t}=t\right) d t=\frac{\mathrm{d}}{\Phi(q)} .
$$

We then obtain from Lemma 1.10

$$
\begin{aligned}
\int_{0}^{\infty} \mathrm{e}^{-q t} \mathbb{E}\left(\exp \left\{-\lambda g_{t}\right\}\right) d t & =\int_{0}^{\infty} \mathrm{e}^{-q t}\left(\mathrm{e}^{-t \lambda} \mathbb{P}\left(g_{t}=t\right)+\int_{[0, t)} \mathrm{e}^{-\lambda s} \bar{\Pi}(t-s) U(d s)\right) d t \\
& =\frac{\mathrm{d}}{\Phi(q+\lambda)}+\int_{0}^{\infty} d t \int_{[0, t)} U(d s) \mathrm{e}^{-q(t-s)} \bar{\Pi}(t-s) \mathrm{e}^{-(\lambda+q) s} \\
& =\frac{\mathrm{d}}{\Phi(q+\lambda)}+\mathcal{L} U(q+\lambda) \mathcal{L} \bar{\Pi}(q) \\
& =\frac{\mathrm{d}}{\Phi(q+\lambda)}+\frac{1}{\Phi(q+\lambda)}\left(\frac{\Phi(q)}{q}-\mathrm{d}\right) .
\end{aligned}
$$

This establishes our claim.

One should note that Lemma 1.11 entails that the law of a subordinator is essentially characterized by that of $g_{\tau}$, where $\tau$ is an independent exponential time. Specifically, if $\sigma^{(1)}$ and $\sigma^{(2)}$ are two subordinators such that, in the obvious notation, $g_{\tau}^{(1)}$ and $g_{\tau}^{(2)}$ have the same law, then there is a constant $c>0$ such that $\Phi^{(1)}=c \Phi^{(2)}$. This observation will be quite useful in the sequel. 


\section{Chapter 2}

\section{Regenerative property}

This chapter is mostly expository; its purpose is to stress the correspondence between a regenerative set, the range of a subordinator, and the set of times when a Markov process visits a fixed point. We refer to Blumenthal [21], Blumenthal and Getoor [23], Dellacherie et al. [44, 45], Kingman [100] and Sharpe [141] for background and much more on this topic.

\section{$2.1 \quad$ Regenerative sets}

The Markov property of a subordinator has a remarkable consequence on its range. First, note that for every $s \geq 0, L_{s}=\inf \left\{t \geq 0: \sigma_{t}>s\right\}$ is an $\left(\mathcal{F}_{t}\right)$-stopping time, and the sigma-fields $\left(\mathcal{M}_{s}=\mathcal{F}_{L_{s}}\right)_{s \geq 0}$ thus form a filtration. Because $L$ is a continuous $\left(\mathcal{M}_{s}\right)$-adapted process that increases exactly on $\mathcal{R}$, the latter is an $\left(\mathcal{M}_{s}\right)$-progressive set. Then fix $s \geq 0$. An application of the Markov property at $L_{s}$ shows that, conditionally on $\left\{L_{s}<\infty\right\}$, the shifted subordinator $\sigma^{\prime}=\left\{\sigma_{L_{s}+t}-\sigma_{L_{s}}, t \geq 0\right\}$ is independent of $\mathcal{M}_{s}$ and has the same law as $\sigma$. Recall also from (1.6) that

$$
\sigma\left(L_{s}\right)=D_{s}=\inf \{t>s: t \in \mathcal{R}\}
$$

is the first-passage time in $\mathcal{R}$ after $s$. We thus see that conditionally on $\left\{D_{s}<\infty\right\}$, the shifted range

$$
\mathcal{R} \circ \theta_{D_{s}}=\left\{v \geq 0: v+D_{s} \in \mathcal{R}\right\}=\overline{\left\{\sigma_{t}^{\prime}: t \geq 0\right\}}
$$

is independent of $\mathcal{M}_{s}$ and is distributed as $\mathcal{R}$. This is usually referred to as the regenerative property of the range. We stress that the regenerative property of $\mathcal{R}$ does not merely hold at the first passage times $D_{s}$, but more generally at any $\left(\mathcal{M}_{s}\right)$ stopping time $S$ which takes values in the subset of points in $\mathcal{R}$ which are not isolated on their right, a.s. on $\{S<\infty\}$. In that case, one can express $S$ in the form $S=\sigma_{T}$, where $T=L_{S}$ is an $\left(\mathcal{F}_{t}\right)$-stopping time. Then conditionally on $\left\{L_{S}<\infty\right\}$, the shifted range $\mathcal{R} \circ \theta_{S}=\{v \geq 0: v+S \in \mathcal{R}\}$ is independent of $\mathcal{M}_{S}=\mathcal{F}_{T}$ and is distributed as $\mathcal{R}$.

The regenerative property of the range of a subordinator motivates the definition of a regenerative set, that has been studied in particular by Krylov and Yushkevich, 
Kingman, Hoffmann-Jørgensen and Maisonneuve. We refer to Fristedt [60] for a detailed survey including a connection with related concepts and a comprehensive list of references.

Consider a probability space endowed with a complete filtration $\left(\mathcal{M}_{t}\right)_{t>0}$. Let $\mathcal{S}$ be a progressively measurable closed subset of $[0, \infty)$ which contains 0 and has no isolated point. We say that $\mathcal{S}$ is a perfect ${ }^{1}$ regenerative set if for every $s \geq 0$, conditionally on $D_{s}:=\inf \{t>s: t \in \mathcal{S}\}<\infty$, the right-hand portion $\mathcal{S} \circ \theta_{D_{s}}$ of $\mathcal{S}$ as viewed from $D_{s}$, is independent of $\mathcal{M}_{D_{s}}$ and has the same distribution $\mathcal{S}$.

We have seen above that the range of a subordinator is a regenerative set; here is the converse.

Theorem 2.1 (Hoffmann-Jørgensen [74], Maisonneuve [109]) Let $\mathcal{S}$ be a regenerative set.

(i) There is a subordinator $\sigma$ such that $\mathcal{S}=\mathcal{R}=\overline{\left\{\sigma_{t}: 0 \leq t<\zeta\right\}}$ a.s., and the inverse $L$ of $\sigma$ is an $\left(\mathcal{M}_{t}\right)$-adapted process.

(ii) If $\widetilde{\sigma}$ is a second subordinator with range $\mathcal{S}$, then there is a real number $c>0$ such that $\widetilde{\sigma}_{t}=\sigma_{c t}$ for all $t \geq 0$, a.s.

We refer to Maisonneuve [109] or Chapter XX in Dellacherie et al. [45] for the proof.

With regards to Theorem 2.1, it will be convenient to use henceforth the notation $\mathcal{R}$ instead of $\mathcal{S}$ to designate a regenerative set. Plainly, if $\widetilde{\sigma}$ is as in Theorem 2.1(ii), then $\widetilde{\Phi}(\lambda)=c \Phi(\lambda)$ in the obvious notation. Hence, among the one-parameter family of subordinators having the range $\mathcal{R}$, there is a unique one for which Laplace exponent satisfies the -arbitrary- normalizing condition

$$
\Phi(1)=1
$$

We refer to $\Phi$ as the Laplace exponent of $\mathcal{R}$

The inverse $L$ of the subordinator $\sigma$ is called the local time on $\mathcal{R}$, it can be constructed explicitly as a function of $\mathcal{R}$ as follows. Recall first from Proposition 1.8 that $\mathcal{R}$ is called heavy if it has a positive Lebesgue measure (or equivalently if the drift coefficient of $\sigma$ is positive) and light otherwise. In the heavy case, one can express the local time as

$$
L_{t}=\mathrm{d}^{-1} m([0, t] \cap \mathcal{R}), \quad t \geq 0
$$

where $m$ stands for the Lebesgue measure. In the light case, Fristedt and Pruitt [61] have obtained a remarkable analogue of Proposition 1.8. Specifically, they have been able to exhibit a deterministic measure $m_{H}$ on $[0, \infty)$ (which is the Hausdorff measure associated with some increasing function) such that

$$
L_{t}=m_{H}([0, t] \cap \mathcal{R}), \quad t \geq 0 .
$$

\footnotetext{
${ }^{1}$ The qualification 'perfect' refers to the absence of isolated points and will be frequently omitted in the sequel in the sense that, for us, a regenerative set has no isolated points. This squares with the fact that compound Poisson processes have been ruled out in this text. For completeness, we mention that a closed random set that has the regenerative property and possesses at least one isolated point with positive probability, is in fact discrete a.s. and can be identified as the range of a compound Poisson process.
} 
We refer to Greenwood and Pitman [68] and Fristedt and Taylor [64] for alternative constructions of the local time on a regenerative set.

We also stress the additive property of the local time: If $S$ is an $\left(\mathcal{M}_{s}\right)$-stopping time which takes values in points in $\mathcal{R}$ with are not isolated on their right, then on $\{S<\infty\}$, the local time $L^{\prime}$ on $\mathcal{R}^{\prime}=\mathcal{R} \circ \theta_{S}$ is given by

$$
L_{t}^{\prime}=L_{S+t}-L_{S} \quad \text { for all } t \geq 0 \text {, a.s. }
$$

\subsection{Connection with Markov processes}

Consider some Polish space $E$ and write $\mathcal{D}$ for the space of càdlàg paths valued in $E$, endowed with Skorohod's topology. Let $X=\left(\Omega, \mathcal{M}, \mathcal{M}_{t}, X_{t}, \theta_{t}, \mathbf{P}^{x}\right)$ be a strong Markov process with sample paths in $\mathcal{D}$. As usual, $\mathbf{P}^{x}$ refers to its law started at $x$, $\theta_{t}$ for the shift operator and $\left(\mathcal{M}_{t}\right)_{t \geq 0}$ for the filtration.

A point $r$ of the state space is regular for itself if

$$
\mathbf{P}^{r}\left(T_{r}=0\right)=1
$$

where $T_{r}=\inf \left\{t>0: X_{t}=r\right\}$ is the first hitting time of $r$. In words, $r$ is regular for itself if the Markov process started at $r$, returns to $r$ at arbitrarily small times, a.s. Applying the Markov property at the first return-time to $r$ after a fixed time $s$, we see that the closure of set of times when $X$ visits $r$,

$$
\mathcal{R}=\overline{\left\{t \geq 0: X_{t}=r\right\}}
$$

is regenerative for $\left(\Omega, \mathcal{M}, \mathcal{M}_{t}, \mathbf{P}^{r}\right)$. (Conversely, it can be proved that any -perfectregenerative set can be viewed as the closed set of times when some Markov process visits a regular point, see Horowitz [76].)

According to Theorem 2.1, $\mathcal{R}$ can thus be viewed as the range of some subordinator $\sigma$. The inverse $L$ of $\sigma$ is a continuous increasing process which increases exactly when $X$ passes at $r$, in the sense that $\operatorname{Supp}(d L)=\mathcal{R}, \mathbf{P}^{r}$-a.s. One calls $L=\left(L_{t}, t \geq 0\right)$ the local time of $X$ at $r$; its existence has been established originally by Blumenthal and Getoor [23], following the pioneering contribution of Lévy in the Brownian case.

The killing rate of the inverse local time has an obvious probabilistic interpretation in terms of the Markov process. One says that $r$ is a transient state if $\mathcal{R}$ is bounded a.s., so that

$$
r \text { is a transient state } \Longleftrightarrow \mathrm{k}>0 \Longleftrightarrow L_{\infty}<\infty \text { a.s. }
$$

More precisely, $L_{\infty}$ has then an exponential distribution with parameter $\mathrm{k}$. In the opposite case, $\mathcal{R}$ is unbounded a.s., and we say that $r$ is a recurrent state.

We next present a simple criterion to decide whether a point is regular for itself, and in that case, give an explicit expression for the Laplace exponent of the inverse local time. This requires some additional assumption of duality type on the Markov process. Typically, suppose that $X=\left(\Omega, \mathcal{M}, \mathcal{M}_{t}, X_{t}, \theta_{t}, \mathbf{P}^{x}\right)$ and $\widehat{X}=\left(\Omega, \widehat{\mathcal{M}}, \widehat{\mathcal{M}}_{t}, \widehat{X}_{t}, \widehat{\theta}_{t}, \widehat{\mathbf{P}}^{x}\right)$ 
are two standard Markov processes with state space $E$. For every $\lambda>0$, the $\lambda$ resolvent operators of $X$ and $\widehat{X}$ are given by

$$
V^{\lambda} f(x)=\mathbf{E}^{x}\left(\int_{0}^{\infty} f\left(X_{t}\right) \mathrm{e}^{-\lambda t} d t\right) \quad, \quad \widehat{V}^{\lambda} f(x)=\widehat{\mathbf{E}}^{x}\left(\int_{0}^{\infty} f\left(\widehat{X}_{t}\right) \mathrm{e}^{-\lambda t} d t\right), \quad x \in E,
$$

where $f \geq 0$ is a generic measurable function on $E$. We recall that $f \geq 0$ is called $\lambda$ excessive with respect to $\left\{V^{\alpha}\right\}$ if $\alpha V^{\alpha+\lambda} f \leq f$ for every $\alpha>0$ and $\lim _{\alpha \rightarrow \infty} \alpha V^{\alpha} f=f$ pointwise.

We suppose that $X$ and $\widehat{X}$ are in duality with respect to some sigma-finite measure $\xi$. That is, the resolvent operators can be expressed in the form

$$
V^{\lambda} f(x)=\int_{E} v^{\lambda}(x, y) f(y) \xi(d y) \quad, \quad \widehat{V}^{\lambda} f(x)=\int_{E} v^{\lambda}(y, x) f(y) \xi(d y) .
$$

Here, $v^{\lambda}: E \times E \rightarrow[0, \infty]$ stands for the version of the resolvent density such that, for every $x \in E$, the function $v^{\lambda}(\cdot, x)$ is $\lambda$-excessive with respect to the resolvent $\left\{V^{\alpha}\right\}$, and the function $v^{\lambda}(x, \cdot)$ is $\lambda$-excessive with respect to the resolvent $\left\{\widehat{V}^{\alpha}\right\}$. Under a rather mild hypothesis on the resolvent density, one has the following simple necessary and sufficient condition for a point to be regular for itself (see e.g. Proposition 7.3 in $[24])$.

Proposition 2.2 Suppose that for every $\lambda>0$ and $y \in E$, the function $x \rightarrow v^{\lambda}(x, y)$ is lower-semicontinuous. Then, for each fixed $r \in E$ and $\lambda>0$, the following assertions are equivalent:

(i) $r$ is regular for itself.

(ii) For every $x \in E, v^{\lambda}(x, r) \leq v^{\lambda}(r, r)<\infty$.

(iii) The function $x \rightarrow v^{\lambda}(x, r)$ is bounded and continuous at $x=r$.

Finally, if these assertions hold, then the Laplace exponent $\Phi$ of the inverse local time at $r$ is given by

$$
\Phi(\lambda)=v^{1}(r, r) / v^{\lambda}(r, r), \quad \lambda>0 .
$$

In the case when the semigroup of $X$ is absolutely continuous with respect to $\xi$, the resolvent density can be expressed in the form

$$
v^{\lambda}(x, y)=\int_{0}^{\infty} \mathrm{e}^{-\lambda t} p_{t}(x, y) d t .
$$

As the Laplace transform of the renewal measure $U$ of the inverse local time at $r$ is $1 / \Phi(\lambda)$, a quantity that is proportional to $v^{\lambda}(r, r)$ by Proposition 2.2 , we see by Laplace inversion that $U$ is absolutely continuous with respect to the Lebesgue measure, with density $u$ given by

$$
u(t)=c p_{t}(r, r), \quad t>0 .
$$

Observe also that in this framework, Proposition 1.9 entails that for each fixed $t>0$, the probability that $t \in \mathcal{R}$, that is that $X_{t}=r$, is proportional to $p_{t}(r, r)$ in the heavy case, and is zero in the light case. Of course, this easy fact can be also checked directly. 
Suppose for instance that $X$ is a real-valued Brownian motion. The resolvent density (with respect to the Lebesgue measure) is

$$
v^{\lambda}(x, y)=\int_{0}^{\infty} \mathrm{e}^{-\lambda t} \frac{1}{\sqrt{2 \pi t}} \exp \left(-\frac{(x-y)^{2}}{2 t}\right) d t=\frac{1}{\sqrt{2 \lambda}} \exp \{-\sqrt{2 \lambda}|x-y|\} .
$$

This quantity depends symmetrically on $x$ and $y$, so the dual process is simply $\widehat{X}=X$. Proposition 2.2 applies and shows that any $r \in \mathbb{R}$ is a regular point for itself, and the Laplace exponent of the inverse local time is always $\Phi(\lambda)=\sqrt{\lambda}$. More generally, when $X$ is a so-called Bessel process of dimension $d \in(0,2)$ (see chapter XI in Revuz and Yor [132]), then $r=0$ is a regular point and the inverse local time at 0 is a stable subordinator with index $\alpha=1-d / 2$. Making use of the results of chapter 5 , we see for instance that the fractal dimension (both lower and upper) of the zero set of a $d$-dimensional Bessel process is $1-d / 2$. Alternatively, when $X$ is a stable Lévy process with index $\beta \in(1,2]$, then any $r \in \mathbb{R}$ is a regular point for itself and the inverse local time is always a stable subordinator with index $\alpha=1-1 / \beta$ (see the forthcoming Proposition 8.1).

We next turn our attention to one of the most important applications of the notion of local time to Markov processes, namely Itô's theory of excursions. This is a vast topic and we shall merely recall the basic result of Itô and refer to the literature for developments (cf. in particular Blumenthal [21], chapter XII in Revuz and Yor [132], chapter 8 in Rogers and Williams [137] and also Rogers [136] for an elementary approach).

Call excursion intervals the maximal open time-intervals on which $X \neq r$. In other words, the excursion intervals are those that appear in the canonical decomposition of the open set $[0, \infty)-\mathcal{R}$. We have already pointed out that those open intervals are precisely of the type $(\sigma(t-), \sigma(t))$ for the $t$ 's such that $\Delta_{t}>0$. Itô used this observation and defined the excursion process $\left(e_{t}, t \geq 0\right)$ of $X$ away from $r$, which is a process valued in the path-space $\mathcal{D}$ given by

$$
e_{t}(s)=\left\{\begin{array}{cc}
X_{\sigma(t-)+s} & \text { if } 0 \leq s<\sigma(t)-\sigma(t-) \\
r & \text { otherwise }
\end{array}\right.
$$

Recall that a point process $\left(\xi_{t}: t \geq 0\right)$ with values in some metric-complete separable space is called a Poisson point process with characteristic measure $\mu$ if for every Borel set $B$, the counting process $N^{B}=$ Card $\left\{t \in[0, \cdot]: \xi_{t} \in B\right\}$ is a Poisson process with intensity $\mu(B)$, and to disjoint Borel sets correspond independent counting processes. We are now able to state Itô's description of the excursions of a Markov process away from a point $r$; we focus for the sake of simplicity on the case when $r$ is a regular recurrent state.

Theorem 2.3 (Itô [82]) When $r$ is a regular recurrent state, the excursion process $\left(e_{t}, t \geq 0\right)$ is a Poisson point process under $\mathbf{P}^{r}$. Its characteristic measure $n$ is called Itô's excursion measure of $X$ away from $r$.

We henceforth suppose that $r$ is a regular recurrent point. The excursion measure yields a very useful expression for the (essentially unique) invariant measure of $X$, 
which is well-known in the context of Markov chains. Specifically, let $\epsilon \in \mathcal{D}$ be a generic path; write $\rho(\epsilon)=\inf \{t>0: \epsilon(t)=r\}$ for its first-return time to $r$. The sigma-finite measure $\mu$ related to the occupation measure under Itô's excursion measure $n$ by

$$
\int f d \mu=\mathrm{d} f(r)+n\left(\int_{0}^{\rho(\epsilon)} f(\epsilon(t)) d t\right)
$$

where $\mathrm{d}$ is the drift coefficient of the inverse local time $\sigma$ and $f \geq 0$ any measurable function, is an invariant measure for $X$. We refer to Getoor [66] or to section XIX.46 in [45] for a proof, and to Maisonneuve [112] for some applications.

Recall that a recurrent Markov process is called positive recurrent if there is an invariant probability measure, and null recurrent otherwise. In our setting, we see that positive recurrence is equivalent to the integrability of the first-return time to $r, \rho$, under Itô's excursion measure. On the other hand, the very definition of the excursion process implies that $\rho\left(e_{t}\right)=\Delta_{t}$, i.e. the durations of the excursion process coincide with the lengths of the jumps of the inverse local time $\sigma$. In particular, the comparison between Theorem 2.3 and Proposition 1.3 shows that the distribution of $\rho$ under $n$ can be identified as the Lévy measure $\Pi$ of $\sigma$ :

$$
n(\rho \in d t)=\Pi(d t) .
$$

In conclusion, we have the equivalence:

$$
X \text { is positive recurrent } \Longleftrightarrow \mathbb{E}\left(\sigma_{1}\right)<\infty \Longleftrightarrow \int_{0}^{\infty} \bar{\Pi}(x) d x<\infty .
$$

We now end this chapter by presenting a brief dictionary in which the main connections between subordinators, local times of Markov processes and regenerative sets are summarized.

$$
\begin{aligned}
& \text { Subordinator } \\
& \text { Local time } \\
& \text { Lifetime } \\
& \sigma_{t}=L_{t}^{-1}=\inf \left\{s: L_{s}>t\right\} \\
& L_{t}=\inf \left\{s: \sigma_{s}>t\right\} \\
& \text { Regenerative set } \\
& \zeta=L_{\infty} \\
& \text { First passage time } \\
& \mathcal{R}=\overline{\left\{t \geq 0: X_{t}=r\right\}}=\overline{\left\{\sigma_{s}: s \in[0, \zeta)\right\}}=\operatorname{Supp}\left(d L_{t}\right) \\
& \text { Last passage time } \\
& \text { Probability } \\
& D_{t}=\inf \{s>t: s \in \mathcal{R}\}=\sigma\left(L_{t}\right) \\
& g_{t}=\sup \{s<t: s \in \mathcal{R}\}=\sigma\left(L_{t}-\right) \\
& \text { Filtration } \\
& \mathbb{P}=\mathbf{P}^{r} \\
& \mathcal{F}_{t}=\mathcal{M}_{\sigma_{t}}
\end{aligned}
$$




\section{Chapter 3}

\section{Asymptotic behaviour of last passage times}

We are concerned with the process $\left(g_{t}: t>0\right)$ of the last passage times in a regenerative set $\mathcal{R}$. When $\mathcal{R}$ is self-similar, $t^{-1} g_{t}$ always has a generalized arcsine law. In the general case, we consider the asymptotic behaviour of $t^{-1} g_{t}$ as $t$ goes to $\infty$, first in distribution, and then pathwise. Special properties of the jump process of a subordinator play a key part in this study.

\subsection{Asymptotic behaviour in distribution}

\subsubsection{The self-similar case}

We say that a regenerative set $\mathcal{R}$ is self-similar if for every $k>0$, it has the same distribution as $k \mathcal{R}$. If we think of $\mathcal{R}$ as the range of a subordinator $\sigma$, this is equivalent to the condition that the Laplace exponent of $\sigma$ is proportional to that of $k \sigma$, i.e. $\Phi(\lambda)=c_{k} \Phi(k \lambda)$ for every $\lambda \geq 0$, where $c_{k}>0$ is some constant that depends only on $k$. Due to the normalization $\Phi(1)=1$, this holds if and only if $\Phi(\lambda)=\lambda^{\alpha}$ for some $\alpha \in[0,1]$, that is if $\sigma$ is a standard stable subordinator of index $\alpha$. The cases $\alpha=0$ and $\alpha=1$ are somewhat degenerate, as they corresponds to the situation where $\mathcal{R}=\{0\}$ and $\mathcal{R}=[0, \infty)$ a.s., respectively; we shall exclude them in the sequel.

Recall that $g_{t}=\sup \{s<t: s \in \mathcal{R}\}$ denotes the last passage time in $\mathcal{R}$ before time $t>0$. When $\mathcal{R}$ is self-similar, the distribution of $t^{-1} g_{t}$ does not depend on $t>0$ and can be given explicitly in terms of $\alpha$.

Proposition 3.1 Suppose that $\Phi(\lambda)=\lambda^{\alpha}$ for some $0<\alpha<1$. Then $g_{1}$ has the so-called generalized arcsine law, that is

$$
\mathbb{P}\left(g_{1} \in d s\right)=\frac{s^{\alpha-1}(1-s)^{-\alpha}}{\Gamma(\alpha) \Gamma(1-\alpha)} d s=\frac{\sin \alpha \pi}{\pi} s^{\alpha-1}(1-s)^{-\alpha} d s \quad(0<s<1) .
$$

For instance, when $\mathcal{R}=\left\{t: B_{t}=0\right\}$ is the zero set of a one-dimensional Brownian motion $B$ started at 0 , we have $\Phi(\lambda)=\sqrt{\lambda}$ (the absence of the usual factor $\sqrt{2}$ is due 
to the normalization (2.1) of the local time) and one gets

$$
\mathbb{P}\left(g_{1} \leq t\right)=\frac{2}{\pi} \arcsin \sqrt{t} \quad(t \in[0,1]) .
$$

This is the celebrated first arcsine theorem of Paul Lévy; see e.g. Exercise III.3.20 in [132] for a direct proof. Proposition 3.1 also applies to the particular cases when one replaces the Brownian motion $B$ by a Bessel process of dimension $d \in(0,2)$ (then $\alpha=1-d / 2$ ), or a stable Lévy process with index $\beta \in(1,2]$ (then $\alpha=1-1 / \beta)$.

We now proceed to the proof of Proposition 3.1.

Proof: The Laplace transform of the renewal measure is given by

$$
\int_{0}^{\infty} \mathrm{e}^{-\lambda x} U(d x)=\lambda^{-\alpha}=\frac{1}{\Gamma(\alpha)} \int_{0}^{\infty} \mathrm{e}^{-\lambda x} x^{\alpha-1} d x
$$

and that of the tail of the Lévy measure by

$$
\int_{0}^{\infty} \mathrm{e}^{-\lambda x} \bar{\Pi}(x) d x=\lambda^{\alpha-1}=\frac{1}{\Gamma(1-\alpha)} \int_{0}^{\infty} \mathrm{e}^{-\lambda x} x^{-\alpha} d x
$$

We conclude by Laplace inversion and Lemma 1.10 .

The distribution of the first-passage time $D_{t}=\inf \{s>t: s \in \mathcal{R}\}$ readily follows from Proposition 3.1 (still in the case when $\Phi(\lambda)=\lambda^{\alpha}$ ). Specifically, for every $0<$ $s<t$, we have

$$
g_{t} \leq s \Longleftrightarrow \mathcal{R} \cap(s, t)=\emptyset \Longleftrightarrow D_{s} \geq t
$$

An application of the scaling property then yields for $t>1$

$$
\mathbb{P}\left(D_{1} \geq t\right)=\mathbb{P}\left(D_{1 / t} \geq 1\right)=\mathbb{P}\left(g_{1} \leq 1 / t\right)=\frac{\sin \alpha \pi}{\pi} \int_{0}^{1 / t} s^{\alpha-1}(1-s)^{-\alpha} d s,
$$

and we deduce that the distribution of $D_{1}$ is given by

$$
\mathbb{P}\left(D_{1} \in d t\right)=\frac{\sin \alpha \pi}{\pi} t^{-1}(t-1)^{-\alpha} d t, \quad t>1 .
$$

Finally we refer to Pitman and Yor $[126,127,129]$ and the references therein for further recent remarkable results about the interval partitions of $[0, \infty)$ induced by self-similar regenerative sets.

\subsubsection{The Dynkin-Lamperti theorem}

We next turn our attention to the asymptotic behaviour of the last passage time in the case when $\mathcal{R}$ is not necessarily self-similar. Informally, the rescaled set $t^{-1} \mathcal{R}$ is the range of the subordinator $t^{-1} \sigma$; its Laplace exponent is thus $\Phi_{t}(q)=\Phi(q / t) / \Phi(1 / t)$, due to (2.1). This quantity converges as $t \rightarrow \infty$ if and only if $\Phi$ is regularly varying at $0+$, and then the limit is $q^{\alpha}$ for some $\alpha \in[0,1]$, that is the Laplace exponent of a stable subordinator with index $\alpha$. In view of Proposition 3.1, one naturally expects that $t^{-1} g_{t}$ should then converge in distribution towards the generalized arcsine law with parameter $\alpha$. The Dynkin-Lamperti theorem not only provides a rigorous setting to this informal argument, but also states a converse. 
Theorem 3.2 (Dynkin [50], Lamperti [106]) The following assertions are equivalent:

(i) $t^{-1} g_{t}$ converges in law as $t \rightarrow \infty$.

(ii) $\lim _{t \rightarrow \infty} t^{-1} \mathbb{E}\left(g_{t}\right)=\alpha \in[0,1]$.

(iii) $\lim _{q \rightarrow 0+} q \Phi^{\prime}(q) / \Phi(q)=\alpha \in[0,1]$.

(iv) $\Phi$ is regularly varying at $0+$ with index $\alpha \in[0,1]$.

Moreover, when these assertions hold, then the limit distribution of $t^{-1} g_{t}$ is the Dirac point mass at 0 (respectively, at 1) for $\alpha=0$ (respectively, $\alpha=1$ ); and for $0<\alpha<1$, the generalized arcsine law of parameter $\alpha$ that appears in Proposition 3.1.

There is also a similar result for small times; more precisely a true statement is obtained after exchanging the rôles of $0+$ and $\infty$. We also mention that, more generally, the limit behaviour in distribution of the pair $\left(g_{t}, D_{t}\right)$ can be studied, using essentially the same arguments as below.

Proof: (i) $\Longrightarrow$ (ii) is obvious as $g_{t} / t \leq 1$.

(ii) $\Longrightarrow$ (iii) On the one hand, we know from Lemma 1.11 that

$$
\int_{0}^{\infty} \mathrm{e}^{-q t} \mathbb{E}\left(g_{t}\right) d t=\frac{\Phi^{\prime}(q)}{q \Phi(q)}
$$

On the other hand, we see by an Abelian theorem that (ii) entails

$$
\lim _{q \rightarrow 0+} q^{2} \int_{0}^{\infty} \mathrm{e}^{-q t} \mathbb{E}\left(g_{t}\right) d t=\alpha .
$$

(iii) $\Longrightarrow$ (iv) When (iii) holds, the logarithmic derivative of $t \rightarrow t^{-\alpha} \Phi(t)$ can be expressed as $t \rightarrow \varepsilon(t) / t$, with $\lim _{t \rightarrow 0+} \varepsilon(t)=0$. That is

$$
t^{-\alpha} \Phi(t)=c \exp \left\{\int_{t}^{1} \frac{\varepsilon(s)}{s} d s\right\} .
$$

According to the representation theorem of slowly varying functions (see e.g. [20]), this shows that $t \rightarrow t^{-\alpha} \Phi(t)$ is slowly varying at $0+$, and hence $\Phi$ is regularly varying at $0+$ with index $\alpha$.

(iv) $\Longrightarrow$ (i) Suppose first that (iv) holds with $0<\alpha<1$. According to Proposition 1.5 , we have

$$
\lim _{t \rightarrow \infty} U(t x) \Phi(1 / t)=\frac{x^{\alpha}}{\Gamma(1+\alpha)} \quad \text { uniformly for } x \in K
$$

and

$$
\lim _{t \rightarrow \infty} \frac{\bar{\Pi}(t x)}{\Phi(1 / t)}=\frac{x^{-\alpha}}{\Gamma(1-\alpha)} \quad \text { uniformly for } x \in K,
$$

where $K$ stands for a generic compact subset on $(0, \infty)$.

Next, fix $0<a<b<1$. According to Lemma 1.10, we have 


$$
\begin{aligned}
\mathbb{P}\left(a t \leq g_{t}<b t\right)=\int_{[a t, b t)} \bar{\Pi}(t-s) d U(s) & =\int_{[a, b)} \bar{\Pi}(t(1-u)) d U(t u) \\
& =\int_{[a, b)} \frac{\bar{\Pi}(t(1-u))}{\Phi(1 / t)} d(U(t u) \Phi(1 / t)) .
\end{aligned}
$$

Applying (3.1) and (3.2), we deduce that

$$
\lim _{t \rightarrow \infty} \mathbb{P}\left(a t \leq g_{t}<b t\right)=\int_{a}^{b} \frac{(1-s)^{-\alpha} s^{\alpha-1}}{\Gamma(\alpha) \Gamma(1-\alpha)} d s .
$$

In words, $t^{-1} g_{t}$ converges in distribution to the generalized arcsine law with parameter $\alpha$.

An easy variation of this argument applies for $\alpha=0$, but not for $\alpha=1$ (the quantity $\Gamma(1-\alpha)$ in $(3.2)$ is then infinite). So suppose that $\alpha=1$, take any $a \in(0,1)$ and observe from Lemma 1.10 that

$$
\mathbb{P}\left(t^{-1} g_{t}<a\right)=\int_{[0, t a)} \bar{\Pi}(t-u) U(d u) \leq \bar{\Pi}(t(1-a)) U(t a) .
$$

A Tauberian theorem applied to the Lévy-Khintchine formula now gives

$$
I(s) \sim s \Phi(1 / s) \quad \text { as } s \rightarrow \infty
$$

where $I$ is the integrated tail of the Lévy measure. In particular $I$ is slowly varying. The inequality

$$
I(s)-I(s / 2)=\int_{s / 2}^{s} \bar{\Pi}(t) d t \geq s \bar{\Pi}(s) / 2
$$

and the fact that $I$ is slowly varying entail that $\bar{\Pi}(s)=o(I(s) / s)=o(\Phi(1 / s))$. Using Proposition 1.4 and (3.3) gives

$$
\lim _{t \rightarrow \infty} \mathbb{P}\left(t^{-1} g_{t}<a\right)=0,
$$

and the proof of Theorem 3.2 is complete.

Theorem 3.2 is essentially an application of the estimates of Proposition 1.4 for the tail of the Lévy measure and the renewal measure. In the same vein, the renewal theorem readily yields the following well-known limit theorem.

Proposition 3.3 Suppose that $\mathbb{E}\left(\sigma_{1}\right)=\mu<\infty$. Then

$$
\lim _{t \rightarrow \infty} \mathbb{P}\left(t-g_{t} \in d s\right)=\frac{1}{\mu} \bar{\Pi}(s) d s, \quad s>0
$$

and

$$
\lim _{t \rightarrow \infty} \mathbb{P}\left(t-g_{t}=0\right)=\mathrm{d} / \mu
$$

Proof: This is an easy application of the renewal theorem (see Proposition 1.6) and Lemma 1.10. 


\section{$3.2 \quad$ Asymptotic sample path behaviour}

The purpose of this section is to investigate the almost-sure asymptotic behaviour of the last-passage-time process; here is the main result (see also [9]).

Theorem 3.4 Let $f:(0, \infty) \rightarrow(0, \infty)$ be a continuous strictly increasing function with $\lim _{t \rightarrow \infty} f(t) / t=0$ and $\liminf _{t \rightarrow \infty} f(t) / f(2 t)>0$. Then, with probability one,

$$
\liminf _{t \rightarrow \infty} g_{t} / f(t)=0 \text { or } \infty
$$

according as the integral

$$
\int_{[1, \infty)} U(f(t)) \Pi(d t)
$$

diverges or converges.

When we specialize Theorem 3.4 to the case when $\mathcal{R}$ is the zero set of a onedimensional Brownian motion, we get $\liminf _{t \rightarrow \infty} g_{t} / f(t)=0$ or $\infty$ a.s. according as the integral $\int^{\infty} \sqrt{f(t) t^{-3}} d t$ diverges or converges. In particular,

$$
\liminf _{t \rightarrow \infty} \frac{g_{t} \log ^{2} t}{t}=0 \quad \text { and } \quad \lim _{t \rightarrow \infty} \frac{g_{t} \log ^{2+\varepsilon} t}{t}=\infty \quad \text { a.s. }
$$

for any $\varepsilon>0$. This result goes back to Chung and Erdős [36], see also Hobson [73] and $\mathrm{Hu}$ and Shi [79] for recent developments in the same vein.

Checking Theorem 3.4 when the killing rate $\mathrm{k}$ is positive, is straightforward. Indeed, $\mathcal{R}$ is then bounded, and so is $g_{t}$ a.s. On the other hand, the renewal measure is also bounded and the integral (3.4) always converges. So with no loss of generality, we may assume henceforth that $\mathrm{k}=0$. The proof of Theorem 3.4 relies on two simple properties of subordinators. Informally, we have to compare the relative size of a subordinator and its jumps. Our first lemma reduces this comparison to that of certain integrals. Recall Proposition 1.3.

Lemma 3.5 For every Borel function $b:[0, \infty) \rightarrow[1, \infty)$, the events

$$
\left\{\Delta_{t}>b\left(\sigma_{t-}\right) \text { infinitely often as } t \rightarrow \infty\right\}
$$

and

$$
\left\{\int_{0}^{\infty} \bar{\Pi} \circ b\left(\sigma_{t}\right) d t=\infty\right\}
$$

coincide up to a set of probability zero.

Proof: This is a variant of the Lévy-Borel-Cantelli lemma. Specifically, the fact that the jump process $\Delta$ is a Poisson point process with characteristic measure $\Pi$ entails that the compensated sum

$$
\sum_{s \leq t} \mathbf{1}_{\left\{\Delta_{s}>b\left(\sigma_{s-}\right)\right\}}-\int_{0}^{t} \bar{\Pi} \circ b\left(\sigma_{s}\right) d s \quad(t \geq 0)
$$


is a martingale. On the event

$$
\left\{\Delta_{t}>b\left(\sigma_{t-}\right) \text { infinitely often as } t \rightarrow \infty\right\} \bigcap\left\{\int_{0}^{\infty} \bar{\Pi} \circ b\left(\sigma_{t}\right) d t<\infty\right\},
$$

this martingale converges to $\infty$; whereas on the event

$$
\left\{\Delta_{t} \leq b\left(\sigma_{t-}\right) \text { for all sufficiently large } t\right\} \bigcap\left\{\int_{0}^{\infty} \bar{\Pi} \circ b\left(\sigma_{t}\right) d t=\infty\right\}
$$

it converges to $-\infty$. As the jumps of this martingale are bounded by 1 , both events have probability zero (see e.g. the corollary on page 484 in [144]).

Motivated by the preceding lemma, we then establish an easy result on the convergence of integrals of a subordinator.

Lemma 3.6 Let $h:[0, \infty) \rightarrow[0, \infty)$ be a decreasing function. The following assertions are equivalent.

$$
\int_{0}^{\infty} h(x) U(d x)<\infty
$$

$$
\mathbb{P}\left(\int_{0}^{\infty} h\left(\sigma_{t}\right) d t<\infty\right)=1
$$

$$
\mathbb{P}\left(\int_{0}^{\infty} h\left(\sigma_{t}\right) d t<\infty\right)>0
$$

Proof: The derivations (i) $\Rightarrow$ (ii) $\Rightarrow$ (iii) are obvious. Suppose that (iii) holds and pick $\varepsilon>0$ and $k>0$ such that

$$
\mathbb{P}\left(\int_{0}^{\infty} h\left(\sigma_{t}\right) d t<k\right)>\varepsilon
$$

Next, consider for every integer $n>0$ the stopping time

$$
T_{n}=\inf \left\{t: \int_{0}^{t} h\left(\sigma_{s}\right) d s \geq k n\right\},
$$

and apply the Markov property (Proposition 1.1) at time $T_{n}$. We see that conditionally on $\left\{T_{n}<\infty\right\}$, the process $\sigma^{\prime}=\sigma_{T_{n}+}$. $-\sigma_{T_{n}}$ is a subordinator distributed as $\sigma$. Then, using the hypothesis that $h$ decreases, we get

$$
\begin{aligned}
\mathbb{P}\left(T_{n+1}=\infty \mid T_{n}<\infty\right) & =\mathbb{P}\left(\int_{T_{n}}^{\infty} h\left(\sigma_{t}\right) d t<k \mid T_{n}<\infty\right) \\
& =\mathbb{P}\left(\int_{0}^{\infty} h\left(\sigma_{t}^{\prime}+\sigma_{T_{n}}\right) d t<k \mid T_{n}<\infty\right) \\
& \geq \mathbb{P}\left(\int_{0}^{\infty} h\left(\sigma_{t}^{\prime}\right) d t<k \mid T_{n}<\infty\right) \\
& =\mathbb{P}\left(\int_{0}^{\infty} h\left(\sigma_{t}\right) d t<k\right)>\varepsilon .
\end{aligned}
$$


This shows that $k^{-1} \int_{0}^{\infty} h\left(\sigma_{t}\right) d t$ is bounded from above by a geometric variable. As a consequence, it has finite expectation and (i) follows.

We point out that when one specializes Lemma 3.6 to the case when $\sigma$ is a stable subordinator with index 1/2, one recovers a result of Donati-Martin, Rajeev and Yor (Theorem 6.2 in [46] and Theorem 1.3 in [131]) on the a.s. convergence of certain integrals involving the Brownian local time. Theorem 3.4 now follows readily from Lemmas 3.5 and 3.6.

Proof of Theorem 3.4: Write $f^{-1}$ for the inverse function of $f$, so $f\left(\Delta_{t}\right)>\sigma_{t-}$ if and only if $\Delta_{t}>f^{-1}\left(\sigma_{t-}\right)$. An immediate combination of Lemmas 3.6 and 3.5 shows that

$$
\mathbb{P}\left(f\left(\Delta_{t}\right)>\sigma_{t-} \text { infinitely often as } t \rightarrow \infty\right)=0 \text { or } 1
$$

according as the integral $\int^{\infty} \bar{\Pi} \circ f^{-1}(x) d U(x)$ converges or diverges. By a change of variables and an integration by parts, the latter is equivalent to the integral (3.4) being finite or infinite. Next, recall that $g_{t}=\sigma\left(L_{t}-\right)$ for all $t \geq 0$ a.s. It follows that $f\left(\Delta_{t}\right)>\sigma_{t-}$ infinitely often as $t \rightarrow \infty$ if and only if $f\left(t-g_{t}\right)>g_{t}$ infinitely often. We deduce that a.s.,

$$
\liminf _{t \rightarrow \infty} g_{t} / f\left(t-g_{t}\right) \geq 1 \text { or } \leq 1
$$

according as (3.4) converges or diverges.

First, assume that (3.4) diverges. By the subadditivity of the renewal function, the same holds when $f$ is replaced by $\varepsilon f$ for an arbitrary $\varepsilon \in(0,1)$. It follows that $\liminf _{t \rightarrow \infty} g_{t} / f\left(t-g_{t}\right)=0$ a.s., and because $f$ increases, we conclude that $\liminf \operatorname{in}_{t \rightarrow \infty} g_{t} / f(t)=0$ a.s.

Finally, assume that (3.4) converges. By the same argument based on the subadditivity of the renewal function as above, we have that $\lim _{t \rightarrow \infty} g_{t} / f\left(t-g_{t}\right)=\infty$ a.s. It is then straightforward to derive from the assumptions $\lim _{t \rightarrow \infty} f(t) / t=0$ and $\liminf _{t \rightarrow \infty} f(t) / f(2 t)>0$ that $\lim _{t \rightarrow \infty} g_{t} / f(t)=\infty$ a.s. (simply distinguish the cases $g_{t} \leq t / 2$ and $\left.g_{t}>t / 2\right)$.

We now conclude this chapter with an interesting application of the techniques developed so far to the case when the regenerative set is given in the form $\mathcal{R}=$ $\overline{\left\{t \geq 0: X_{t}=r\right\}}$, where $X$ is some Markov process started from a regular point $r$. Theorem 3.2 provides a necessary and sufficient condition for $g_{t} / t$ to converge in probability; and it is natural to ask whether the convergence then holds almost surely. To this end, the equivalence

$$
\lim _{t \rightarrow \infty} g_{t} / t=0 \quad \text { a.s. } \quad \Longleftrightarrow \quad r \text { is a transient state }
$$

is obvious (if $r$ is a recurrent state, then $g_{t}=t$ infinitely often). The problem of the convergence towards 1 is less obvious. Its solution is essentially a variation of a result of Kesten on the asymptotic behaviour of the largest step of increasing random walks. 
Proposition 3.7 (Kesten [97]) The following assertions are equivalent:

(i) $\lim _{t \rightarrow \infty} g_{t} / t=1$ a.s.

(ii) $\mathbb{P}\left(\liminf _{t \rightarrow \infty} g_{t} / t>0\right)>0$.

(iii) The Markov process $X$ is positive recurrent.

Proof: (i) $\Leftrightarrow$ (ii) It is immediate to see that (i) holds if and only if for every $\varepsilon>0$, $\Delta_{t} \leq \varepsilon \sigma_{t-}$ for all sufficiently large $t$, a.s. By Lemmas 3.5 and 3.6, we deduce that

$$
\text { (i) } \Longleftrightarrow \int^{\infty} \bar{\Pi}(\varepsilon t) d U(t)<\infty \quad \text { for every } \varepsilon>0 \text {. }
$$

Similarly, (ii) holds if and only if the event $\left\{\Delta_{t} \leq k \sigma_{t-}\right.$ for all sufficiently large $\left.t\right\}$ has positive probability for some $k<\infty$. Again by Lemmas 3.5 and 3.6, we deduce that

$$
\text { (ii) } \Longleftrightarrow \int^{\infty} \bar{\Pi}(k t) d U(t)<\infty \quad \text { for some } k<\infty \text {. }
$$

Because the renewal function is subadditive, an integration by parts now shows that (i) and (ii) are equivalent.

(i) $\Leftrightarrow$ (iii) Let us exclude the degenerate case when $\sigma$ is a pure drift, and recall from Proposition 1.4 that then $U(t) \asymp t / I(t)$ as $t \rightarrow \infty$ where $I$ stands for the integrated tail of the Lévy measure. On the other hand, we know from the preceding argument that

$$
\text { (i) } \Longleftrightarrow \int^{\infty} \bar{\Pi}(t) d U(t)<\infty \Longleftrightarrow \int^{\infty} \frac{t \Pi(d t)}{I(t)}<\infty,
$$

where the second equivalence follows from an integration by parts.

Recall from (2.3) that $X$ is positive recurrent if and only if $\mathbb{E}\left(\sigma_{1}\right)<\infty$, that is if and only if $I(\infty)=\int_{0}^{\infty} \bar{\Pi}(t) d t=\int_{(0, \infty)} t \Pi(d t)<\infty$. Because $I$ is an increasing function, it is plain that (i) holds in this case.

We next suppose that (i) holds. It is immediately checked that the mapping $t \rightarrow t / I(t)$ increases, and the convergence of the preceding integral thus forces $t \bar{\Pi}(t)=$ $o(I(t))$. An integration by parts shows that

$$
\int^{\infty} \bar{\Pi}(t)\left(\frac{1}{I(t)}-\frac{t \bar{\Pi}(t)}{I(t)^{2}}\right) d t<\infty
$$

and hence we must have $\int^{\infty} \bar{\Pi}(t) I^{-1}(t) d t<\infty$. The latter is clearly equivalent to $I(\infty)<\infty$, that is to (iii).

In the positive recurrent case, an application of Lemmas 3.5 and 3.6 and the renewal theorem (Proposition 1.6) shows that the sample path behaviour of the last passage time process is specified as follows: For every increasing function $f:(0, \infty) \rightarrow$ $(0, \infty)$

$$
\mathbb{P}\left(t-g_{t}>f(t) \text { infinitely often as } t \rightarrow \infty\right)=0 \text { or } 1
$$

according as the integral $\int^{\infty} \bar{\Pi} \circ f(t) d t$ converges or diverges. 


\section{Chapter 4}

\section{Rates of growth of local time}

We present the remarkable law of the iterated logarithm for the local time due to Fristedt and Pruitt, and also investigate the modulus of continuity of the local time on a path. The independence and stationarity of the increments of a subordinator are the key to the proper application of the Borel-Cantelli lemma.

\subsection{Law of the iterated logarithm}

The main result of this section is the following version of the law of the iterated logarithm for local times.

Theorem 4.1 (Fristedt and Pruitt $[61]^{1}$ ) There exists a positive and finite constant $c_{\Phi}$ such that

$$
\limsup _{t \rightarrow 0+} \frac{L_{t} \Phi\left(t^{-1} \log \log \Phi\left(t^{-1}\right)\right)}{\log \log \Phi\left(t^{-1}\right)}=c_{\Phi} \quad \text { a.s. }
$$

The exact value of $c_{\Phi}$ does not seem to be known explicitly in general. When $\Phi$ is regularly varying with index $\alpha \in[0,1]$ at $\infty$, then $c_{\Phi}=c_{\alpha}$, where

$$
c_{\alpha}=\alpha^{-\alpha}(1-\alpha)^{-(1-\alpha)},
$$

with the convention $0^{-0}=1$; see Barlow, Perkins and Taylor [5], or [8]. The sharpest result related to Theorem 4.1 is in Pruitt [130].

There is also a version of Theorem 4.1 for large times, which follows from a simple variation of the arguments for small times. Specifically, suppose that the killing rate is $\mathrm{k}=0$. Then there exists $c_{\Phi}^{\prime} \in(0, \infty)$ such that

$$
\limsup _{t \rightarrow \infty} \frac{L_{t} \Phi\left(t^{-1} \log \left|\log \Phi\left(t^{-1}\right)\right|\right)}{\log \left|\log \Phi\left(t^{-1}\right)\right|}=c_{\Phi}^{\prime} \quad \text { a.s. }
$$

\footnotetext{
${ }^{1}$ Theorem 4.1 is slightly more explicit than the result stated in [61]. Specifically, the normalizing function there is the inverse function of $t \rightarrow \varphi\left(t^{-1} \log \log \varphi\left(t^{-1}\right)\right)^{-1} \log \log \varphi\left(t^{-1}\right)$, where $\varphi$ denotes the inverse function of $\Phi$. However, after some tedious calculation, one can check that the normalizing function in [61] and that in Theorem 4.1 are of the same order, and therefore the two statements agree.
} 
When $L$ is the local time at a regular point for some recurrent Markov process, the ergodic theorem asserts that if $A$ is a positive additive functional associated with a measure $\mu$ with finite mass, then $A_{t} \sim \mu(E) L_{t}$ as $t \rightarrow \infty$, a.s. A law of the iterated logarithm for $A$ thus follows from (4.2). Further developments in the direction of a second order law, were made recently by Csáki et al. [39], Marcus and Rosen [115, 116], Bertoin [8], Khoshnevisan [98]...

The proof of Theorem 4.1 relies on two technical lemmas. We write

$$
f(t)=\frac{\log \log \Phi\left(t^{-1}\right)}{\Phi\left(t^{-1} \log \log \Phi\left(t^{-1}\right)\right)}, \quad t \text { small enough, }
$$

and denote the inverse function of $\Phi$ by $\varphi$.

Lemma 4.2 For every integer $n \geq 2$, put

$$
t_{n}=\frac{\log n}{\varphi\left(\mathrm{e}^{n} \log n\right)} \quad, \quad a_{n}=f\left(t_{n}\right) .
$$

(i) The sequence $\left(t_{n}: n \geq 2\right)$ decreases, and we have $a_{n} \sim \mathrm{e}^{-n}$.

(ii) The series $\Sigma \mathbb{P}\left(L_{t_{n}}>3 a_{n}\right)$ converges

Proof: (i) The first assertion follows readily from the fact that $\varphi$ is convex and increasing. On the one hand, since $\Phi$ increases, we have for $n \geq 3$

$$
\Phi\left(t_{n}^{-1}\right)=\Phi\left(\varphi\left(\mathrm{e}^{n} \log n\right) / \log n\right) \leq \Phi\left(\varphi\left(\mathrm{e}^{n} \log n\right)\right)=\mathrm{e}^{n} \log n .
$$

On the other hand, since $\Phi$ is concave, we have for $n \geq 3$

$$
\Phi\left(t_{n}^{-1}\right)=\Phi\left(\varphi\left(\mathrm{e}^{n} \log n\right) / \log n\right) \geq \Phi\left(\varphi\left(\mathrm{e}^{n} \log n\right)\right) / \log n=\mathrm{e}^{n} .
$$

This entails

$$
\log \log \Phi\left(t_{n}^{-1}\right) \sim \log n
$$

and then

$$
t_{n}^{-1} \log \log \Phi\left(t_{n}^{-1}\right) \sim \varphi\left(\mathrm{e}^{n} \log n\right) .
$$

Note that if $\alpha_{n} \sim \beta_{n}$, then $\Phi\left(\alpha_{n}\right) \sim \Phi\left(\beta_{n}\right)$ (because $\Phi$ is concave and increasing). We deduce that

$$
\Phi\left(t_{n}^{-1} \log \log \Phi\left(t_{n}^{-1}\right)\right) \sim \mathrm{e}^{n} \log n,
$$

and our assertion follows from (4.3). by

(ii) The probability of the event $\left\{L_{t_{n}}>3 a_{n}\right\}=\left\{\sigma_{3 a_{n}}<t_{n}\right\}$ is bounded from above

$$
\exp \left\{\lambda t_{n}\right\} \mathbb{E}\left(\exp \left\{-\lambda \sigma_{3 a_{n}}\right\}\right)=\exp \left\{\lambda t_{n}-3 a_{n} \Phi(\lambda)\right\}
$$

for every $\lambda \geq 0$. We choose $\lambda=\varphi\left(\mathrm{e}^{n} \log n\right)$; so $\Phi(\lambda)=\mathrm{e}^{n} \log n$ and $\lambda t_{n}=\log n$. Our statement follows now from (i).

Lemma 4.3 For every integer $n \geq 2$, put

$$
s_{n}=\frac{2 \log n}{\varphi\left(2 \exp \left\{n^{2}\right\} \log n\right)} \quad, \quad b_{n}=f\left(s_{n}\right) .
$$

(i) We have $b_{n} \sim \exp \left\{-n^{2}\right\}$.

(ii) The series $\Sigma \mathbb{P}\left(\sigma\left(b_{n} / 3\right)<2 s_{n} / 3\right)$ diverges 
Proof: (i) Just note that $s_{n}=t_{n^{2}}$ and apply Lemma 4.2(i).

(ii) For every $b, s$ and $\lambda \geq 0$, we have

$$
\mathbb{P}\left(\sigma_{b} \geq s\right) \leq\left(1-\mathrm{e}^{-\lambda s}\right)^{-1} \mathbb{E}\left(1-\exp \left\{-\lambda \sigma_{b}\right\}\right)
$$

which entails

$$
\mathbb{P}\left(\sigma_{b}<s\right) \geq \frac{\mathrm{e}^{-b \Phi(\lambda)}-\mathrm{e}^{-\lambda s}}{1-\mathrm{e}^{-\lambda s}} .
$$

Apply this to $b=b_{n} / 3, s=2 s_{n} / 3$ and $\lambda=\varphi\left(2 \exp \left\{n^{2}\right\} \log n\right)$, and observe that then $\Phi(\lambda)=2 \exp \left\{n^{2}\right\} \log n, \lambda s=\frac{4}{3} \log n$ and $b \Phi(\lambda) \sim \frac{2}{3} \log n$ (by (i)). In particular $\mathrm{e}^{-b \Phi(\lambda)} \geq n^{-3 / 4}$ for every sufficiently large $n$; we thus obtain

$$
2 \mathbb{P}\left(\sigma\left(b_{n} / 3\right)<2 s_{n} / 3\right) \geq \frac{n^{-3 / 4}-n^{-4 / 3}}{1-n^{-4 / 3}},
$$

and our claim follows.

We are now able to establish the law of the iterated logarithm, using a standard method based on the Borel-Cantelli lemma.

Proof of Theorem 4.1: 1. To prove the upper-bound, we use the notation of Lemma 4.2. Take any $t \in\left[t_{n+1}, t_{n}\right]$, so, provided that $n$ is large enough

$$
f(t) \geq \frac{\log \log \Phi\left(t_{n}^{-1}\right)}{\Phi\left(t_{n+1}^{-1} \log \log \Phi\left(t_{n+1}^{-1}\right)\right)}
$$

(because $\Phi$ increases). By (4.3), the numerator is equivalent to $\log n$, and, by (4.4), the denumerator to $\mathrm{e}^{n+1} \log (n+1)$. By Lemma 4.2, we thus have

$$
\limsup _{t \rightarrow 0+} f\left(t_{n}\right) / f(t) \leq \mathrm{e} .
$$

On the other hand, an application of the Borel-Cantelli to Lemma 4.2 shows that

$$
\limsup _{n \rightarrow \infty} L_{t_{n}} / f\left(t_{n}\right) \leq 3 \quad \text { a.s. }
$$

and we deduce that

$$
\limsup _{t \rightarrow 0+} \frac{L_{t}}{f(t)} \leq\left(\limsup _{n \rightarrow \infty} \frac{L_{t_{n}}}{f\left(t_{n}\right)}\right)\left(\limsup _{t \rightarrow 0+} \frac{f\left(t_{n}\right)}{f(t)}\right) \leq 3 \mathrm{e} \quad \text { a.s. }
$$

2. To prove the lower-bound, we use the notation of Lemma 4.3 and observe that the sequence $\left(b_{n}, n \geq 2\right)$ decreases ultimately (by Lemma 4.3(i)). First, by Lemma 4.3(ii), we have

$$
\sum \mathbb{P}\left(\sigma\left(b_{n} / 3\right)-\sigma\left(b_{n+1} / 3\right)<2 s_{n} / 3\right) \geq \sum \mathbb{P}\left(\sigma\left(b_{n} / 3\right)<2 s_{n} / 3\right)=\infty ;
$$

so by the Borel-Cantelli lemma for independent events,

$$
\liminf _{n \rightarrow \infty} \frac{\sigma\left(b_{n} / 3\right)-\sigma\left(b_{n+1} / 3\right)}{s_{n}} \leq \frac{2}{3}
$$


If we admit for a while that

$$
\limsup _{n \rightarrow \infty} \frac{\sigma\left(b_{n+1} / 3\right)}{s_{n}} \leq \frac{1}{4}
$$

we can conclude that

$$
\liminf _{n \rightarrow \infty} \frac{\sigma\left(b_{n} / 3\right)}{s_{n}}<\frac{11}{12}
$$

This implies that the set $\{s: \sigma(f(s) / 3)<s\}$ is unbounded a.s. Plainly, the same then holds for $\left\{s: L_{s}>f(s) / 3\right\}$, and as a consequence:

$$
\limsup _{t \rightarrow 0+} L_{t} / f(t) \geq 1 / 3 \quad \text { a.s. }
$$

Now we establish (4.6). The obvious inequality (which holds for any $\lambda>0$ )

$$
\mathbb{P}\left(\sigma\left(b_{n+1} / 3\right)>s_{n} / 4\right) \leq\left(1-\exp \left\{-\lambda s_{n} / 4\right\}\right)^{-1} \mathbb{E}\left(1-\exp \left\{-\lambda \sigma\left(b_{n+1} / 3\right)\right\}\right)
$$

entails for the choice

that

$$
\lambda=\varphi\left(2 \exp \left\{n^{2}\right\} \log n\right)=\frac{2 \log n}{s_{n}}
$$

$$
\mathbb{P}\left(\sigma\left(b_{n+1} / 3\right)>s_{n} / 4\right) \leq \frac{2 b_{n+1} \exp \left\{n^{2}\right\} \log n}{3\left(1-\exp \left\{-\frac{1}{2} \log n\right\}\right)}
$$

By Lemma 4.3(i), the numerator is bounded from above for every sufficiently large $n$ by

$$
3 \exp \left\{n^{2}-(n+1)^{2}\right\} \log n \leq \mathrm{e}^{-n}
$$

and the denumerator is bounded away from 0 . We deduce that the series

$$
\sum \mathbb{P}\left(\sigma\left(b_{n+1} / 3\right)>s_{n} / 4\right)
$$

converges, and the Borel-Cantelli lemma entails (4.6). The proof of (4.7) is now complete.

3. The two preceding parts show that

$$
\limsup _{t \rightarrow 0+} L_{t} / f(t) \in[1 / 3,3 \mathrm{e}] \quad \text { a.s. }
$$

By the Blumenthal zero-one law, it must be a constant number $c_{\Phi}$, a.s.

To conclude this section, we mention that the independence and homogeneity of the increments of the inverse local time are also very useful in investigating the class of lower functions for the local time. We now state without proof the main result in that field, which has been proven independently by Fristedt and Skorohod. See [57], [67], or Theorem III.9 in [11], where the result is given in terms of the rate of growth of the subordinator.

Proposition 4.4 (i) When $\mathrm{d}>0$, one has $\lim _{t \rightarrow 0+} L_{t} / t=1 / \mathrm{d}$ a.s.

(ii) When $\mathrm{d}=0$ and $f:[0, \infty) \rightarrow[0, \infty)$ is an increasing function such that $t \rightarrow f(t) / t$ decreases, one has

$$
\liminf _{t \rightarrow 0+} L_{t} / f(t)=0 \quad \text { a.s. } \Longleftrightarrow \int_{0+} f(x) \Pi(d x)=\infty .
$$

Moreover, if these assertions fail, then $\lim _{t \rightarrow 0+} L_{t} / f(t)=\infty$ a.s. 


\subsection{Modulus of continuity}

Once a law of the iterated logarithm has been established for a continuous process, it is natural to look for information on its modulus of continuity. Again we have a general result that holds for any local time of a Markov process.

Theorem 4.5 For every $T>0$, we have a.s.

$$
\limsup _{t \rightarrow 0+}\left\{\sup _{0 \leq \tau \leq T} \frac{\left(L_{\tau+t}-L_{\tau}\right) \Phi\left(t^{-1} \log \Phi\left(t^{-1}\right)\right)}{\log \Phi\left(t^{-1}\right)}\right\} \leq 12
$$

and

$$
\liminf _{t \rightarrow 0+}\left\{\sup _{0 \leq \tau \leq T} \frac{\left(L_{\tau+t}-L_{\tau}\right) \Phi\left(t^{-1} \log \Phi\left(t^{-1}\right)\right)}{\log \Phi\left(t^{-1}\right)}\right\} \geq 1 / 6
$$

Theorem 4.5 has been obtained in a less explicit form by Fristedt and Pruitt [62], following an earlier work of Hawkes [69] in the stable case. The bounds 1/6 and 12 are clearly not optimal, and a much more precise result is available under the condition that $\Phi$ is regularly varying with index $\alpha \in[0,1]$ at $\infty$ : In that case, one has a.s.

$$
\lim _{t \rightarrow 0+}\left\{\sup _{0 \leq \tau \leq T} \frac{\left(L_{\tau+t}-L_{\tau}\right) \Phi\left(t^{-1} \log \Phi\left(t^{-1}\right)\right)}{\log \Phi\left(t^{-1}\right)}\right\}=c_{\Phi}
$$

where $c_{\Phi}$ is the constant that appears in Theorem 4.1; see e.g. [8]. Whether or not this identity holds in any case is an open problem.

To start with, we write

$$
g(t)=\frac{\log \Phi\left(t^{-1}\right)}{\Phi\left(t^{-1} \log \Phi\left(t^{-1}\right)\right)}, \quad t \text { small enough, }
$$

and recall that $\varphi$ stands for the inverse function of $\Phi$. We then introduce for every integer $n \geq 2$ :

$$
t(n)=\frac{n}{\varphi\left(n \mathrm{e}^{n}\right)} \quad, \quad a(n)=g(t(n))
$$

Lemma 4.6 (i) The sequence $(t(n): n \geq 2)$ decreases. Moreover we have:

$$
\log \Phi\left(t(n)^{-1}\right) \sim n \quad, \quad \Phi\left(t(n)^{-1} \log \Phi\left(t(n)^{-1}\right)\right) \sim n \mathrm{e}^{n} \quad, \quad a_{n} \sim \mathrm{e}^{-n} .
$$

(ii) For $n$ large enough and any $t \in[t(n+1), t(n)]$, we have

$$
a(n) / 3 \leq g(t) \leq 3 a(n+1) .
$$

Proof: (i) follows from an argument similar to that in Lemma 4.2.

(ii) Since $\Phi$ increases, we have

$$
g(t) \geq \frac{\log \Phi\left(t(n)^{-1}\right)}{\Phi\left(t(n+1)^{-1} \log \Phi\left(t(n+1)^{-1}\right)\right)} .
$$

We know from (i) that the numerator is equivalent to $n$, and the denumerator to $(n+1) \mathrm{e}^{n+1}$. Using (i) again, we deduce that for $n$ large enough, $g(t) \geq a(n) / 3$. The proof of the second inequality is similar.

Next, we establish the following upper bound. 
Lemma 4.7 We have for every $\rho>0$

$$
\limsup _{t \rightarrow 0+}\left\{\sup _{0 \leq \tau \leq \sigma_{\rho}}\left(L_{\tau+t}-L_{\tau}\right) / g(t)\right\} \leq 12, \quad \text { a.s. }
$$

Proof: Consider for every $n \in \mathbb{N}$ and every integer $j=0,1, \cdots,[\rho / a(n)]$ the event

$$
A_{j n}=\left\{\sigma_{(j+3) a(n)}-\sigma_{j a(n)} \leq t(n)\right\} .
$$

By the Markov property of $\sigma$, we have for every $\lambda>0$

$$
\begin{aligned}
\mathbb{P}\left(A_{j n}\right)=\mathbb{P}\left(\sigma_{3 a(n)} \leq t(n)\right) & \leq \exp \{\lambda t(n)\} \mathbb{E}\left(\exp \left\{-\lambda \sigma_{3 a(n)}\right\}\right) \\
& =\exp \{\lambda t(n)-3 a(n) \Phi(\lambda)\} .
\end{aligned}
$$

The choice $\lambda=\varphi\left(n \mathrm{e}^{n}\right)$ together with Lemma 4.6(i) yield

$$
\mathbb{P}\left(A_{j n}\right) \leq \exp \left\{n-3 n \mathrm{e}^{n} a(n)\right\}=o\left(\mathrm{e}^{-2 n}\right) ;
$$

so that (using again Lemma 4.6(i)) $\mathbb{P}\left(\bigcup_{j} A_{j n}\right)=o\left(\mathrm{e}^{-n}\right)$. Hence $\sum_{n} \mathbb{P}\left(\bigcup_{j} A_{j n}\right)<\infty$. We conclude that $\sigma_{(j+3) a(n)}-\sigma_{j a(n)}>t(n)$ for all large enough $n$ and all integers $j \leq[\rho / a(n)]$, a.s.

We now work on the event that

$$
\limsup _{t \rightarrow 0+}\left\{\sup _{0 \leq \tau \leq \sigma_{\rho}}\left(L_{\tau+t}-L_{\tau}\right) / g(t)\right\}>12 .
$$

Then, for some arbitrarily large $n$, we can find $t \in[t(n+1), t(n)]$ and $\tau \in\left[0, \sigma_{\rho}\right]$ such that $L_{\tau+t}-L_{\tau}>12 g(t)$. On the other hand, we have $(j-1) a(n) \leq L_{\tau} \leq j a(n)$ for some integer $j \leq[\rho / a(n)]$. By Lemma 4.6(ii), this implies

$$
L_{\tau+t}>(j-1) a(n)+12 g(t) \geq(j-1) a(n)+4 a(n)=(j+3) a(n) ;
$$

and therefore we then have both

$$
\sigma_{j a(n)} \geq \tau \quad \text { and } \quad \sigma_{(j+3) a(n)}<\tau+t .
$$

In conclusion, we must have $\sigma_{(j+3) a(n)}-\sigma_{j a(n)}<t \leq t(n)$; and we know that the probability of the latter event goes to zero as $n \rightarrow \infty$.

The first part of Theorem 4.5 derives from Lemma 4.7 by an immediate argument of monotonicity. Similarly, the second part is a consequence of the following lemma.

Lemma 4.8 We have for every $\eta>0$ :

$$
\liminf _{t \rightarrow 0+}\left\{\sup _{0 \leq \tau \leq \sigma_{\eta}}\left(L_{\tau+t}-L_{\tau}\right) / g(t)\right\} \geq 1 / 2, \quad \text { a.s. }
$$


Proof: We keep the notation of Lemma 4.6. Consider for every $n \in \mathbb{N}$ and every integer $j=0,1, \cdots,[\eta / a(n)]$ the event

$$
B_{j n}=\left\{\sigma_{(j+1) a(n) / 2}-\sigma_{j a(n) / 2} \geq t(n)\right\} .
$$

By the independence and stationarity of the increments of $\sigma$, we have

$$
\mathbb{P}\left(\bigcap_{j} B_{j n}\right)=\mathbb{P}\left(B_{0, n}\right)^{[\eta / a(n)]} \leq \exp \left\{-\frac{\eta}{a(n)}\left(1-\mathbb{P}\left(B_{0 n}\right)\right)\right\}
$$

To estimate the right-hand side, we apply (4.5) with $b=a(n) / 2, s=t(n)$ and $\lambda=\varphi\left(n \mathrm{e}^{n}\right)$, so $\Phi(\lambda)=n \mathrm{e}^{n}$. Using Lemma 4.6(i), we get

$$
1-\mathbb{P}\left(B_{0 n}\right)=\mathbb{P}\left(\sigma_{a(n) / 2}<t(n)\right) \leq \frac{\exp \{-2 n / 3\}-\exp \{-n\}}{1-\exp \{-n\}} \sim \exp \{-2 n / 3\} .
$$

Applying Lemma 4.6(i) again, we deduce that

$$
\mathbb{P}\left(\bigcap_{j} B_{j n}\right)=O(\exp \{-\eta \exp \{n / 2\}\}) .
$$

and the right-hand side induces a summable series.

Applying the Borel-Cantelli lemma, this entails that a.s., for every sufficiently large integer $n$, we are able to pick an integer $j \in\{0,1, \cdots,[\eta / a(n)]\}$ such that

$$
\sigma_{(j+1) a(n) / 2}-\sigma_{j a(n) / 2}<t(n) .
$$

Writing $\tau(n)=\sigma_{j a(n) / 2}$, we thus have $L_{\tau(n)}=j a(n) / 2$ and $L_{\tau(n)+t(n)}>(j+1) a(n) / 2$. This forces

$$
L_{\tau(n)+t(n)}-L_{\tau(n)}>a(n) / 2=g(t(n)) / 2 .
$$

As a consequence, for every $t \in[t(n+1), t(n)]$, Lemma 4.6(ii) and an obvious argument of monotonicity yield

$$
L_{\tau(n+1)+t}-L_{\tau(n+1)}>g(t(n+1)) / 2 \geq g(t) / 6
$$

which establishes the lemma.

The law of the iterated logarithm specifies the rate of growth of the local time at the origin of times. By the regenerative property and the additivity of the local time, we see that for any stopping time $T$ which takes its values in the subset of points in $\mathcal{R}$ which are not isolated on their right, the rate of growth of $L$ at time $T$ is the same as at the origin. Theorem 4.5 can be combined with a condensation argument due to Orey and Taylor [124] to investigate the maximal rate of growth on a path. More precisely, it is immediate from the first part of Theorem 4.5 that

$$
\limsup _{t \rightarrow 0+} \frac{\left(L_{\tau+t}-L_{\tau}\right) \Phi\left(t^{-1} \log \Phi\left(t^{-1}\right)\right)}{\log \Phi\left(t^{-1}\right)}<12, \quad \text { for all } \tau \geq 0,
$$


and the second part, combined with the condensation argument (cf. [124] for details), yields that a.s.

$$
\limsup _{t \rightarrow 0+} \frac{\left(L_{\tau+t}-L_{\tau}\right) \Phi\left(t^{-1} \log \Phi\left(t^{-1}\right)\right)}{\log \Phi\left(t^{-1}\right)} \geq 1 / 2, \quad \text { for some } \tau \geq 0 .
$$

An instant $\tau$ for which the preceding lower bound holds, is referred to as a rapid point for the local time, in the terminology of Kahane [90]. Adapting arguments of Orey and Taylor [124] for Brownian motion, Laurence Marsalle [118] has obtained interesting results about the Hausdorff dimension of the set of fast points when $\Phi$ is regularly varying at $\infty$.

It is also natural to investigate the minimal rate of growth of the local time at instants $\tau \in \mathcal{R}$ which are not isolated on their right $\mathcal{R}$ (otherwise the rate of growth is plainly zero). To this end, Marsalle [118] (extending earlier results of Fristedt [59] in the stable case) has shown recently that under some rather mild conditions on the Laplace exponent $\Phi$, the minimal rate of growth has the same order as $1 / \Phi(1 / t)$. Specifically, one has a.s.

$$
\limsup _{t \rightarrow 0+}\left(L_{\tau+t}-L_{\tau}\right) \Phi(1 / t)>0 \quad \text { for every } \tau \in \mathcal{R} \text { not isolated on its right. , }
$$

and

$$
\limsup _{t \rightarrow 0+}\left(L_{\tau+t}-L_{\tau}\right) \Phi(1 / t)<\infty \quad \text { for some } \tau \geq 0 .
$$

An instant $\tau$ which fulfils the preceding conditions is referred to as a slow point.

Finally, we mention that functional (i.e. à la Strassen) laws of the iterated logarithm for certain local times have been obtained by Marcus and Rosen [115], Csáki et al. [40] and Gantert and Zeitouni [65]. 


\section{Chapter 5}

\section{Geometric properties of regenerative sets}

This chapter is concerned with two geometric aspects of regenerative sets. We first discuss fractal dimensions and then consider the intersection with a given Borel set. The intersection of two independent regenerative sets receives special attention.

\subsection{Fractal dimensions}

\subsubsection{Box-counting dimension}

The box-counting dimension is perhaps the simplest notion amongst the variety of fractal dimensions in use; see Falconer [52]. For every non-empty bounded subset $F \subseteq[0, \infty)$, let $N_{\varepsilon}(F)$ be the smallest number of intervals of length (at most) $\varepsilon>0$ which can cover $F$. The lower and upper box-counting dimensions of $F$ are defined as

$$
\underline{\operatorname{dim}}_{\mathrm{B}}(F)=\liminf _{\varepsilon \rightarrow 0+} \frac{\log N_{\varepsilon}(F)}{\log 1 / \varepsilon} \quad, \quad \overline{\operatorname{dim}}_{\mathrm{B}}(F)=\limsup _{\varepsilon \rightarrow 0+} \frac{\log N_{\varepsilon}(F)}{\log 1 / \varepsilon},
$$

respectively. When these two quantities are equal, their common value is referred to as the box dimension (or also the Minkowski dimension) of $F$.

Following Blumenthal and Getoor [22], we next introduce the so-called lower and upper indices of the Laplace exponent $\Phi$

$$
\begin{aligned}
& \underline{\operatorname{ind}}(\Phi)=\sup \left\{\rho>0: \lim _{\lambda \rightarrow \infty} \Phi(\lambda) \lambda^{-\rho}=\infty\right\}=\liminf _{\lambda \rightarrow \infty} \frac{\log \Phi(\lambda)}{\log \lambda} \\
& \overline{\operatorname{ind}}(\Phi)=\inf \left\{\rho>0: \lim _{\lambda \rightarrow \infty} \Phi(\lambda) \lambda^{-\rho}=0\right\}=\limsup _{\lambda \rightarrow \infty} \frac{\log \Phi(\lambda)}{\log \lambda}
\end{aligned}
$$

with the usual convention $\sup \emptyset=0$. For instance, in the stable case $\Phi(\lambda)=\lambda^{\alpha}$, the lower and upper indices both equal $\alpha$; and for a Gamma process, both the lower and upper indices are zero. Making use of Proposition 1.4, it is easy to exhibit a Laplace exponent such that ind $(\Phi)=a$ and $\overline{\text { ind }}(\Phi)=b$ for arbitrary $0 \leq a \leq b \leq 1$. 
Theorem 5.1 We have a.s. for every $t>0$

$$
\underline{\operatorname{dim}}_{\mathrm{B}}(\mathcal{R} \cap[0, t])=\underline{\operatorname{ind}}(\Phi) \quad \text { and } \quad \overline{\operatorname{dim}}_{\mathrm{B}}(\mathcal{R} \cap[0, t])=\overline{\operatorname{ind}}(\Phi) .
$$

Proof: The argument for the upper dimension is essentially a variation of that for the lower dimension, and we shall merely consider the latter. As we are concerned with a local path property of subordinators, there is no loss of generality in assuming that the killing rate is $\mathrm{k}=0$. Fix $\varepsilon>0$ and introduce by induction the following sequence of finite stopping times: $T(0, \varepsilon)=0$ and

$$
T(n+1, \varepsilon)=\inf \left\{t>T(n, \varepsilon): \sigma_{t}-\sigma_{T(n, \varepsilon)}>\varepsilon\right\}, \quad n=0,1, \cdots
$$

Because the points $\sigma_{T(0, \varepsilon)}, \sigma_{T(1, \varepsilon)}, \cdots$ are at distance at least $\varepsilon$ from each others, we see that for every fixed $t>0$, if $T(n, \varepsilon) \leq t$, then the minimal number of intervals of length $\varepsilon$ that is needed to cover $\mathcal{R} \cap[0, t]$ cannot be less than $n+1$. On the other hand, it is clear from the construction that the intervals $\left[\sigma_{T(n, \varepsilon)}, \sigma_{T(n, \varepsilon)}+\varepsilon\right]$ have length $\varepsilon$ and do cover $\mathcal{R}$. We conclude that

$$
N_{\varepsilon}(\mathcal{R} \cap[0, t])=\operatorname{Card}\left\{n \in \mathbb{N}: \sigma_{T(n, \varepsilon)} \leq t\right\}
$$

Next, introduce an independent exponential time $\tau$ with parameter 1 . The Markov property of $\sigma$ applied at time $T(n, \varepsilon)$ and the lack of memory of the exponential law entail that

$$
\begin{aligned}
\mathbb{P}\left(\sigma_{T(n+1, \varepsilon)} \leq \tau \mid \sigma_{T(n, \varepsilon)} \leq \tau\right) & =\mathbb{P}\left(\sigma_{T(n+1, \varepsilon)}-\sigma_{T(n, \varepsilon)} \leq \tau-\sigma_{T(n, \varepsilon)} \mid \sigma_{T(n, \varepsilon)} \leq \tau\right) \\
& =\mathbb{P}\left(\sigma_{T(1, \varepsilon)} \leq \tau\right) .
\end{aligned}
$$

In other words, the random variable in (5.1) has a geometric distribution with parameter $\mathbb{P}\left(\sigma_{T(1, \varepsilon)} \leq \tau\right)=\mathbb{P}\left(g_{\tau} \geq \varepsilon\right)$, i.e.

$$
\mathbb{P}\left(N_{\varepsilon}(\mathcal{R} \cap[0, \tau])>n\right)=\left(1-\mathbb{P}\left(g_{\tau}<\varepsilon\right)\right)^{n} .
$$

In order to estimate the left-hand side, recall from Lemma 1.11 that the Laplace transform of $g_{\tau}$ is $\Phi(1) / \Phi(1+\cdot)$. It follows from the same argument based on the Tauberian theorem of de Haan and Stadtmüller that we used in the proof of Proposition 1.4, that

$$
\mathbb{P}\left(g_{\tau}<\varepsilon\right) \asymp 1 / \Phi(1 / \varepsilon), \quad(\varepsilon \rightarrow 0+) .
$$

Pick first $\rho>$ ind $(\Phi)$, so (by (5.3)) there is a sequence of positive real numbers $\varepsilon_{n} \downarrow 0$ with $\lim _{n \rightarrow \infty} \varepsilon_{n}^{-\rho} \mathbb{P}\left(g_{\tau}<\varepsilon_{n}\right)=\infty$. It now follows from (5.2) that

$$
\lim _{n \rightarrow \infty} \mathbb{P}\left(N_{\varepsilon_{n}}(\mathcal{R} \cap[0, \tau])>\varepsilon_{n}^{-\rho}\right)=0,
$$

and this forces (by Fatou's lemma)

$$
\liminf _{\varepsilon \rightarrow 0+} \frac{\log N_{\varepsilon}(\mathcal{R} \cap[0, \tau])}{\log 1 / \varepsilon} \leq \rho \quad \text { a.s. }
$$

We have thus proven the upper bound $\underline{\operatorname{dim}}_{B}(\mathcal{R} \cap[0, t]) \leq \underline{\text { ind }}(\Phi)$ a.s. 
To establish the converse lower bound, we may suppose that ind $(\Phi)>0$ since otherwise there is nothing to prove. Then pick $0<\rho<\underline{\text { ind }}(\Phi)$ and note that the series $\sum 2^{n \rho} / \Phi\left(2^{n}\right)$ converges. We deduce from (5.2) and (5.3) that

$$
\sum_{n=0}^{\infty} \mathbb{P}\left(N_{2^{-n}}(\mathcal{R} \cap[0, \tau]) \leq 2^{n \rho}\right)<\infty
$$

so by the Borel-Cantelli lemma and an immediate argument of monotonicity

$$
\liminf _{\varepsilon \rightarrow 0+} \frac{\log N_{\varepsilon}(\mathcal{R} \cap[0, \tau])}{\log 1 / \varepsilon} \geq \rho \quad \text { a.s. }
$$

This shows that $\underline{\operatorname{dim}}_{\mathrm{B}}(\mathcal{R} \cap[0, t]) \geq \underline{\text { ind }}(\Phi)$ a.s.

\subsubsection{Hausdorff and packing dimensions}

Lower and upper box-counting dimensions are attractively simple notions which are rather easy to work with in practice. However they are not always relevant in discussing fractal dimension, due to the following fact (see Proposition 3.4 in [52]): The closure $\bar{F}$ of a set $F$ has the same lower and upper box-counting dimensions as $F$. In particular, a countable dense subset of $[0,1]$ has box-dimension 1 , which is a rather disappointing feature.

This motivated the definition of modified box-counting dimensions (see Falconer [52], section 3.3):

$$
\begin{aligned}
& \underline{\operatorname{dim}}_{\mathrm{MB}}(F)=\inf \left\{\sup _{i} \underline{\operatorname{dim}}_{\mathrm{B}}\left(F_{i}\right): F \subseteq \bigcup_{i=1}^{\infty} F_{i}\right\}, \\
& \overline{\operatorname{dim}}_{\mathrm{MB}}(F)=\inf \left\{\sup _{i} \overline{\operatorname{dim}}_{\mathrm{B}}\left(F_{i}\right): F \subseteq \bigcup_{i=1}^{\infty} F_{i}\right\} .
\end{aligned}
$$

It is clear that in general

$$
\underline{\operatorname{dim}}_{\mathrm{MB}}(F) \leq \underline{\operatorname{dim}}_{\mathrm{B}}(F) \text { and } \overline{\operatorname{dim}}_{\mathrm{MB}}(F) \leq \overline{\operatorname{dim}}_{\mathrm{B}}(F),
$$

and these inequalities can be strict. Nonetheless, the box dimension and its modified version always agree for regenerative sets.

Lemma 5.2 We have a.s. for every $t>0$

$\underline{\operatorname{dim}}_{\mathrm{B}}(\mathcal{R} \cap[0, t])=\underline{\operatorname{dim}}_{\mathrm{MB}}(\mathcal{R} \cap[0, t]) \quad$ and $\quad \overline{\operatorname{dim}}_{\mathrm{B}}(\mathcal{R} \cap[0, t])=\overline{\operatorname{dim}}_{\mathrm{MB}}(\mathcal{R} \cap[0, t])$.

Proof: The random set $\mathcal{R} \cap[0, t]$ is compact and an immediate application of the Markov property shows that

$\underline{\operatorname{dim}}_{\mathrm{B}}(\mathcal{R} \cap[0, t] \cap V)=\underline{\operatorname{dim}}_{\mathrm{B}}(\mathcal{R} \cap[0, t]) \quad, \quad \overline{\operatorname{dim}}_{\mathrm{B}}(\mathcal{R} \cap[0, t] \cap V)=\overline{\operatorname{dim}}_{\mathrm{B}}(\mathcal{R} \cap[0, t])$

for all open sets $V$ that intersect $\mathcal{R} \cap[0, t]$. Our claim follows from Proposition 3.6 in Falconer [52]. 
Taylor and Tricot [148] introduced the so-called packing dimension $\operatorname{dim}_{\mathrm{P}}$, which in fact coincides with the upper modified box-counting dimension $\overline{\operatorname{dim}}_{\mathrm{MB}}$; see Proposition 3.8 in [52]. Combining Lemma 5.2 and Theorem 5.1 thus identifies the packing dimension of a regenerative set with the upper index of its Laplace exponent, which is a special case of a general result of Taylor [147] on the packing dimension of the image of a Lévy process. We refer to Fristedt and Taylor [63] for further results on the packing measure of the range of a subordinator.

We next turn our attention to the so-called Hausdorff dimension; let us first briefly introduce this notion and refer to Rogers [133] for a complete account. Fix $\rho>0$. For every subset $F \subseteq[0, \infty)$ and every $\varepsilon>0$, denote by $\mathcal{C}(\varepsilon)$ the set of all the coverings $C=\left\{I_{i}, i \in \mathcal{I}\right\}$ of $E$ with intervals $I_{i}$ of length $\left|I_{i}\right|<\varepsilon$ (here $\mathcal{I}$ stands for a generic at most countable set of indices). Then introduce

$$
m_{\varepsilon}^{\rho}(F)=\inf _{C \in \mathcal{C}(\varepsilon)} \sum_{i \in I}\left|I_{i}\right|^{\rho} .
$$

Plainly $m_{\varepsilon}^{\rho}(F)$ increases as $\varepsilon$ decreases to $0+$, and the limit is denoted by

$$
m^{\rho}(F)=\lim _{\varepsilon>0} \inf _{C \in \mathcal{C}(\varepsilon)} \sum_{i \in I}\left|I_{i}\right|^{\rho} \in[0, \infty] .
$$

It can be shown that the mapping $F \rightarrow m^{\rho}(F)$ defines a measure on Borel sets, called the $\rho$-dimensional Hausdorff measure. It should be clear that when $F$ is fixed, the mapping $\rho \rightarrow m^{\rho}(F)$ decreases. Moreover, it is easy to see that if $m^{\rho}(F)=0$ then $m^{\rho^{\prime}}(F)=0$ for every $\rho^{\prime}>\rho$; and if $m^{\rho}(F)>0$ then $m^{\rho^{\prime}}(F)=\infty$ for every $\rho^{\prime}<\rho$. The critical value

$$
\operatorname{dim}_{\mathrm{H}}(F)=\sup \left\{\rho>0: m^{\rho}(F)<\infty\right\}=\inf \left\{\rho>0: m^{\rho}(F)=0\right\},
$$

is called the Hausdorff dimension of $F$. We now identify the Hausdorff dimension of $\mathcal{R}$ with the lower index of its Laplace exponent.

Corollary 5.3 (Horowitz [73]) We have for every $t>0 \operatorname{dim}_{\mathrm{H}}(\mathcal{R} \cap[0, t])=\underline{\text { ind }}(\Phi)$ a.s.

Proof: The upper bound follows from Theorem 5.1, Lemma 5.2 and the obvious fact that

$$
\operatorname{dim}_{\mathrm{H}}(F) \leq \underline{\operatorname{dim}}_{\mathrm{B}}(F)
$$

for all bounded sets.

To prove the lower bound, we may suppose that ind $(\Phi)>0$ since otherwise there is nothing to prove. The argument is based on the fact that the local time is a.s. Höldercontinuous with exponent $\rho$ on every compact time interval, for every $\rho<\underline{\text { ind }}(\Phi)$. To establish the latter assertion, note first by an application of the Markov property of $\sigma$ at $L_{t}$ that for every $p>0$ and $s, t \geq 0$ :

$$
\mathbb{E}\left(\left(L_{t+s}-L_{t}\right)^{p}\right) \leq \mathbb{E}\left(L_{s}^{p}\right)
$$

It follows that

$$
\mathbb{E}\left(\left(L_{t+s}-L_{t}\right)^{p}\right) \leq p \int_{0}^{\infty} x^{p-1} \mathbb{P}\left(L_{s}>x\right) d x=p \int_{0}^{\infty} x^{p-1} \mathbb{P}\left(\sigma_{x} \leq s\right) d x .
$$


Using the obvious inequality

$$
\mathbb{P}\left(\sigma_{x} \leq s\right) \leq \mathrm{e} \mathbb{E}\left(\exp \left\{-s^{-1} \sigma_{x}\right\}\right)=\exp \left\{1-x \Phi\left(s^{-1}\right)\right\}
$$

we get

$$
\mathbb{E}\left(\left(L_{t+s}-L_{t}\right)^{p}\right) \leq \mathrm{e} \Gamma(p+1) \Phi\left(s^{-1}\right)^{-p} .
$$

The Hölder-continuity now derives from Kolmogorov's criterion and the very definition of the lower index.

Next, consider a covering of $\mathcal{R} \cap[0, t]$ by finitely many intervals $\left[a_{0}, b_{0}\right], \cdots,\left[a_{n}, b_{n}\right]$, where $a_{0} \leq b_{0} \leq \cdots \leq a_{n} \leq b_{n}$ (there is no loss of generality in focussing on finite coverages, because $\mathcal{R} \cap[0, t]$ is compact). Observe that $L_{b_{i-1}}=L_{a_{i}}$ for $i=1, \cdots, n$. Since $L$ is a.s. Hölder continuous with exponent $\rho$ on $[0,1]$, we deduce that

$$
\sum_{i=0}^{n}\left(b_{i}-a_{i}\right)^{\rho} \geq K \sum_{i=0}^{n}\left(L_{b_{i}}-L_{a_{i}}\right)=K L_{b_{n}} \geq K L_{t}>0 \quad \text { a.s. }
$$

where $K>0$ is a certain random variable. This shows that the $\rho$-Hausdorff measure of $\mathcal{R} \cap[0, t]$ is positive a.s., so its Hausdorff dimension is at least $\rho$.

To summarize the main results of this section, there are two natural fractal dimensions -which may coincide- associated with a regenerative set. The lower dimension agrees both with the Hausdorff dimension and the lower (modified) box-counting dimension; it is given by the lower index of the Laplace exponent. The upper dimension agrees both with the packing dimension and the upper (modified) box-counting dimension; it is given by the upper index of the Laplace exponent.

There exist many further results in the literature about Hausdorff dimension and subordinators; see section III.5 in [11] and [58] and references therein. To this end, we also recall that Fristedt and Pruitt [61] have been able to specify the exact Hausdorff measure of the range; which provides a remarkable refinement of the result of Horowitz. In a different direction, the multifractal structure of the occupation measure of a stable subordinator has been recently considered by $\mathrm{Hu}$ and Taylor [78].

\subsection{Intersections with a regenerative set}

\subsubsection{Equilibrium measure and capacity}

We are concerned with the probability that a regenerative set $\mathcal{R}$ intersects a given (deterministic) Borel set $B$. As $\mathcal{R}$ only differs from $\left\{\sigma_{t}: t>0\right\}$, the set of points that are visited by the subordinator $\sigma$, by at most countably many points, it is readily seen that

$$
\mathbb{P}(\mathcal{R} \cap B \neq \emptyset)=\mathbb{P}\left(\sigma_{t} \in B \text { for some } t>0\right) .
$$

This connection enables us to investigate the left-hand-side using the classical potential theory for Markov processes; see Chapter VI in Blumenthal and Getoor [23], Berg and Forst [6], and the references therein. To this end, it will be convenient to use the 
notation $\mathbb{P}^{x}$ for the law of the subordinator started from $x \in \mathbb{R}$, viz. the distribution of $x+\sigma$ under $\mathbb{P}=\mathbb{P}^{0}$.

For the sake of simplicity, we will assume that the renewal measure is absolutely continuous and that there is a version of the renewal density that is continuous on $(0, \infty)$. As a matter of fact, the results of this section hold more generally under the sole assumption of absolute continuity for the renewal measure; the continuity hypothesis for the renewal density just enables us to circumvent some technical difficulties inherent to the general case. The probability that a bounded Borel set $B$ is hit by $\sigma$ can be expressed in terms of renewal densities and the so-called equilibrium measure of $B$ as follows (cf. Theorem VI(2.8) in [23]).

Proposition 5.4 Suppose that $U$ is absolutely continuous with a continuous density on $(0, \infty)$, and write $u(t)$ for the version of $U(d t) / d t$ such that $u \equiv 0$ on $(-\infty, 0]$ and $u$ is continuous on $(0, \infty)$. Let $B \subseteq(-\infty, \infty)$ be a bounded Borel set. There is a Radon measure $\mu_{B}$, called the equilibrium measure of $B$, with $\operatorname{Supp} \mu_{B} \subseteq \bar{B}$, and such that for every $x \in(-\infty, \infty)$

$$
\mathbb{P}^{x}\left(\sigma_{t} \in B \text { for some } t>0\right)=\int_{(-\infty, \infty)} u(y-x) \mu_{B}(d y) .
$$

Proof: The argument is a variation of that of Chung (cf. Chapter 5 in [35]). Fix $x$ and introduce the last-passage time in $B$,

$$
\gamma=\sup \left\{t>0: \sigma_{t} \in B\right\}
$$

and note that $\sigma_{\gamma_{-}} \in \bar{B}$ whenever $0<\gamma<\infty$. Then consider for every $\varepsilon>0$ and every bounded continuous function $f: \mathbb{R} \rightarrow[0, \infty)$ the quantity

$$
I(\varepsilon)=\varepsilon^{-1} \mathbb{E}^{x}\left(\int_{0}^{\infty} f\left(\sigma_{t}\right) \mathbf{1}_{\{\gamma \in(t, t+\varepsilon)\}} d t\right) .
$$

The continuity of $f$ and the identity

$$
I(\varepsilon)=\mathbb{E}^{x}\left(\varepsilon^{-1} \int_{(\gamma-\varepsilon)^{+}}^{\gamma} f\left(\sigma_{t}\right) d t\right)
$$

make clear that

$$
\lim _{\varepsilon \rightarrow 0+} I(\varepsilon)=\mathbb{E}^{x}\left(f\left(\sigma_{\gamma-}\right), 0<\gamma<\infty\right) .
$$

On the other hand, an application of the Markov property shows that

$$
I(\varepsilon)=\mathbb{E}^{x}\left(\int_{0}^{\infty} f\left(\sigma_{t}\right) \varepsilon^{-1} \psi_{\varepsilon}\left(\sigma_{t}\right) d t\right)=\int_{-\infty}^{\infty} f(y) u(y-x) \varepsilon^{-1} \psi_{\varepsilon}(y) d y,
$$

with $\psi_{\varepsilon}(y)=\mathbb{P}^{y}(0<\gamma<\varepsilon)$.

It is readily seen from the resolvent equation (cf. [11] on page 23) that $u$ is positive on $(0, \infty)$. First take the function $f$ in the form

$$
f(y)=\left\{\begin{array}{cc}
g(y) / u(y-x) & \text { if } y>x \\
0 & \text { otherwise }
\end{array}\right.
$$


where $g$ is a continuous function. As $x$ is arbitrary, we see from (5.4) and (5.5) that the measure $\varepsilon^{-1} \psi_{\varepsilon}(y) d y$ converges weakly towards some Radon measure, say $\mu_{B}$. We then deduce that

$$
\mathbb{P}^{x}\left(\sigma_{\gamma-} \in d y, 0<\gamma<\infty\right)=u(y-x) \mu_{B}(d y)
$$

(recall that $u$ is continuous except at 0 and that $u(0)=0$ ). In particular $\mu_{B}$ has support in $\bar{B}$ and

$$
\mathbb{P}^{x}\left(\sigma_{t} \in B \text { for some } t>0\right)=\mathbb{P}^{x}(0<\gamma<\infty)=\int_{-\infty}^{\infty} u(y-x) \mu_{B}(d y),
$$

which establishes our claim.

The total mass of the equilibrium measure is called the capacity of $B$, and is denoted by

$$
\operatorname{Cap}(B)=\mu_{B}(\mathbb{R})=\mu_{B}(\bar{B}) .
$$

The set $B$ is called polar if it has zero capacity, i.e. its equilibrium measure is trivial. We see from Proposition 5.4 that $B$ is polar if and only if for every starting point $x \in \mathbb{R}$, the subordinator $\sigma$ never visits $B$ at any positive instant. The capacity can also be expressed as

$$
\operatorname{Cap}(B)=\sup \left\{\mu(\mathbb{R}): \mu(\mathbb{R}-B)=0 \text { and } \int_{\mathbb{R}} u(x-y) \mu(d y) \leq 1\right\}
$$

see Blumenthal and Getoor [23] on page 286. As an immediate consequence, one obtains the following characterization of Borel sets $B \subseteq(0, \infty)$ that do not intersect a regenerative set $\mathcal{R}$ :

$\mathbb{P}(B \cap \mathcal{R}=\emptyset)=1 \Longleftrightarrow \sup _{x \in \mathbb{R}} U \mu(x)=\infty \quad \forall \mu$ probability measure with $\mu(B)=1$,

where $U \mu(x)=\int u(y-x) \mu(d y)$.

\subsubsection{Dimension criteria}

The preceding characterization of polar sets is not always easy to apply, as it requires precise information on the renewal density. Our purpose in this section is to present more handy criteria in terms of the Hausdorff dimension (recall section 2.3). We refer to Hawkes [70] for further results connecting the polarity of sets and Hausdorff measures.

In order to present a simple test for non-intersection, we need first to estimate the probability that $\mathcal{R}$ intersects a given interval.

Lemma 5.5 The following bounds hold for every $0<a<b$

$$
\frac{U(b)-U(a)}{U(b-a)} \leq \mathbb{P}(\mathcal{R} \cap[a, b] \neq \emptyset) \leq \frac{U(2 b-a)-U(a)}{U(b-a)} .
$$


Proof: Applying the Markov property at $D_{a}=\inf \{x>a: x \in \mathcal{R}\}=\sigma_{L_{a}}$, we get

$$
\begin{aligned}
U(b)-U(a)=\mathbb{E}\left(\int_{L_{a}}^{\infty} \mathbf{1}_{\left\{\sigma_{t} \in(a, b]\right\}} d t\right) & =\int_{[a, b]} \mathbb{P}\left(\sigma_{L_{a}} \in d x\right) \mathbb{E}\left(\int_{0}^{\infty} \mathbf{1}_{\left\{\sigma_{t} \in(a-x, b-x]\right\}} d t\right) \\
& =\int_{[a, b]} \mathbb{P}\left(D_{a} \in d x\right) U(b-x) \\
& \leq \mathbb{P}\left(D_{a} \leq b\right) U(b-a) .
\end{aligned}
$$

Since the events $\left\{D_{a} \leq b\right\}$ and $\{\mathcal{R} \cap(a, b] \neq \emptyset\}$ coincide, the lower bound is proven.

A similar argument yields the upper-bound. More precisely

$$
\begin{aligned}
U(2 b-a)-U(a) & =\int_{[a, 2 b-a]} \mathbb{P}\left(D_{a} \in d x\right) U(2 b-a-x) \\
& \geq \int_{[a, b]} \mathbb{P}\left(D_{a} \in d x\right) U(2 b-a-x) \geq \mathbb{P}\left(D_{a} \leq b\right) U(b-a) .
\end{aligned}
$$

This entails

$$
\mathbb{P}(\mathcal{R} \cap(a, b] \neq \emptyset) \leq \frac{U(2 b-a)-U(a)}{U(b-a)},
$$

and since the renewal function is continuous, our claim follows.

Proposition 5.6 (Orey [123]) Suppose that the renewal measure has a locally bounded density $u$ on $(0, \infty)$. Let $B \subseteq(0, \infty)$ with $\operatorname{dim}_{\mathrm{H}}(B)<1-\overline{\operatorname{ind}}(\Phi)$. Then $\mathcal{R} \cap B=\emptyset$ a.s.

Proof: As $\operatorname{dim}_{\mathrm{H}}(B)<1-\overline{\operatorname{ind}}(\Phi)$, there is $\rho<1-\overline{\operatorname{ind}}(\Phi)$ such that the $\rho$ dimensional Hausdorff measure of $B$ is zero. This means that for every $\varepsilon>0$, one can cover $B$ with a family of intervals $\left(\left[a_{i}, b_{i}\right]: i \in \mathcal{I}\right)$ such that

$$
\sum_{i \in I}\left|b_{i}-a_{i}\right|^{\rho} \leq \varepsilon
$$

We then invoke Lemma 5.5 to get

$$
\mathbb{P}(\mathcal{R} \cap B \neq \emptyset) \leq \sum_{\mathcal{I}} \mathbb{P}\left(\mathcal{R} \cap\left[a_{i}, b_{i}\right] \neq \emptyset\right) \leq \sum_{\mathcal{I}} \frac{U\left(2 b_{i}-a_{i}\right)-U\left(a_{i}\right)}{U\left(b_{i}-a_{i}\right)}
$$

With no loss of generality, we may (and will) suppose that for some $c>1,1 / c \leq a_{i}<$ $b_{i} \leq c$ for every $i$. As $U$ is Lipschitz-continuous on $[1 / c, 2 c]$, the right-hand side in the ultimate displayed equation is less than or equal to

$$
M \sum_{\mathcal{I}} \frac{b_{i}-a_{i}}{U\left(b_{i}-a_{i}\right)}
$$

for some finite constant number $M$.

By Proposition 1.4, we know that there is a constant number $k>0$ such that $1 / U(t) \leq k \Phi(1 / t)$. The very definition of the upper index entails that $\Phi(1 / t)=$ $o\left(t^{\rho-1}\right)$. We conclude that

$$
\mathbb{P}(\mathcal{R} \cap B \neq \emptyset) \leq C \sum_{i \in I}\left|b_{i}-a_{i}\right|^{\rho}
$$


and by (5.7), the right-hand side can be made as small as we wish.

We then give a test for intersection with positive probability.

Proposition 5.7 (Hawkes [70]) Suppose that the renewal measure has a decreasing density $u$ on $(0, \infty)$ with respect to the Lebesgue measure. Let $B \subseteq(0, \infty)$ with $\operatorname{dim}_{\mathrm{H}}(B)>1-\underline{\operatorname{ind}}(\Phi)$. Then $\mathbb{P}(\mathcal{R} \cap B \neq \emptyset)>0$.

Proposition 5.7 follows from (5.6) and the following variation of Frostman's lemma.

Lemma 5.8 Under the hypotheses of Proposition 5.7, there is a probability measure $\mu$ with compact support $K \subseteq B$ such that $\mu * u$ is a bounded function.

Proof: Pick $\rho$ strictly between $1-\underline{\text { ind }}(\Phi)$ and $\operatorname{dim}_{\mathrm{H}}(B)$. According to Frostman's lemma (see e.g. Theorem 4.13 in [52] and its proof), there is a probability measure $\mu$ with compact support $K \subseteq B$ such that

$$
\sup _{x \geq 0} \int_{[0, \infty)}|y-x|^{-\rho} \mu(d y)<\infty .
$$

Applying Proposition 1.4 and the hypothesis that the renewal density $u$ decreases, we get

$$
u(t) \leq \frac{U(t)}{t} \leq \frac{c}{t \Phi(1 / t)}
$$

On the other hand, we know from the very definition of the lower index that $\Phi(1 / t)$ is bounded from below by $t^{\rho-1}$ for all small enough $t>0$. In conclusion $u(t)=O\left(t^{-\rho}\right)$ and our claim follows.

We point out that, since the Laplace transform of the renewal measure is $1 / \Phi$, the renewal density exists and is decreasing if and only if $\lambda / \Phi(\lambda)$ is the Laplace exponent of some subordinator (this is seen by an integration by parts), and then Propositions 5.6 and 5.7 are relevant. For instance, recall that the zero set of a $d$-dimensional Bessel process $(0<d<2)$ can be viewed as the range of a stable subordinator with index $1-d / 2$. We deduce that a $d$-dimensional Bessel process never vanishes a.s. on a time-set $B \subseteq(0, \infty)$ with Hausdorff dimension strictly less than $d / 2$, whereas it vanishes with positive probability on a time-set with Hausdorff dimension strictly greater than $d / 2$.

\subsubsection{Intersection of independent regenerative sets}

We finally consider the intersection of two independent regenerative sets, say $\mathcal{R}^{(1)}$ and $\mathcal{R}^{(2)}$. It should be clear that the closed random set $\mathcal{R}=\mathcal{R}^{(1)} \cap \mathcal{R}^{(2)}$ inherits the regenerative property, and our main concern is then to characterize its distribution.

The case when both $\mathcal{R}^{(1)}$ and $\mathcal{R}^{(2)}$ are heavy is straightforward. Specifically, write $\mathrm{d}^{(1)}$ and $\mathrm{d}^{(2)}$ for the positive drift coefficients of $\mathcal{R}^{(1)}$ and $\mathcal{R}^{(2)}$, respectively, and recall 
that the renewal densities $u^{(1)}$ and $u^{(2)}$ are continuous and positive on $[0, \infty)$ (cf. Proposition 1.9). Because $\mathcal{R}^{(1)}$ and $\mathcal{R}^{(2)}$ are independent, we have for every $x \geq 0$

$$
\mathbb{P}(x \in \mathcal{R})=\mathbb{P}\left(x \in \mathcal{R}^{(1)}\right) \mathbb{P}\left(x \in \mathcal{R}^{(2)}\right)=\mathrm{d}^{(1)} \mathrm{d}^{(2)} u^{(1)}(x) u^{(2)}(x) .
$$

The right-hand side is a continuous everywhere positive function of $x$; we conclude by an application of Proposition 1.9 that $\mathcal{R}$ is a heavy regenerative set whose renewal density is proportional to $u^{(1)} u^{(2)}$. We present below a more general result.

Proposition 5.9 (Hawkes [71]) Let $\mathcal{R}^{(1)}$ and $\mathcal{R}^{(2)}$ be two independent regenerative sets and $\mathcal{R}=\mathcal{R}^{(1)} \cap \mathcal{R}^{(2)}$. Suppose that $\mathcal{R}^{(1)}$ and $\mathcal{R}^{(2)}$ both possess renewal densities $u^{(1)}$ and $u^{(2)}$ which are continuous and positive on $(0, \infty)$, and that $\mathcal{R}$ does not reduce to $\{0\}$ a.s. Then $\mathcal{R}$ has a renewal density given by $u=c u^{(1)} u^{(2)}$, where $c>0$ the constant of normalization.

Proof: The idea of the proof is the same as for Proposition 5.4. We assume first that $\mathcal{R}^{(1)}$ is bounded, and hence so is $\mathcal{R}$. Introduce the last passage times

$$
\gamma^{(1)}=\sup \left\{t>0: \sigma_{t}^{(1)} \in \mathcal{R}^{(2)}\right\} \quad, \quad \gamma^{(2)}=\sup \left\{t>0: \sigma_{t}^{(2)} \in \mathcal{R}^{(1)}\right\}
$$

which are positive and finite by assumption. Note also that the largest point of $\mathcal{R}$ can be expressed as the common value $g_{\infty}=\sigma^{(1)}\left(\gamma^{(1)}-\right)=\sigma^{(2)}\left(\gamma^{(2)}-\right)$. Take a bounded continuous function $f:[0, \infty) \times[0, \infty) \rightarrow[0, \infty)$, and consider for every $\varepsilon>0$ the quantity

$$
I(\varepsilon)=\mathbb{E}\left(\varepsilon^{-2} \int_{0}^{\infty} d s \int_{0}^{\infty} d t f\left(\sigma_{s}^{(1)}, \sigma_{t}^{(2)}\right) \mathbf{1}_{\left\{\gamma^{(1)} \in(s, s+\varepsilon), \gamma^{(2)} \in(t, t+\varepsilon)\right\}}\right) .
$$

On the one hand, we can write $I(\varepsilon)$ as

$$
\mathbb{E}\left(\varepsilon^{-2} \int_{\gamma^{(1)}-\varepsilon}^{\gamma^{(1)}} d s \int_{\gamma^{(2)}-\varepsilon}^{\gamma^{(2)}} d t f\left(\sigma_{s}^{(1)}, \sigma_{t}^{(2)}\right)\right)
$$

and then apply the theorem of dominated convergence to get

$$
\lim _{\varepsilon \rightarrow 0+} I(\varepsilon)=\mathbb{E}\left(f\left(g_{\infty}, g_{\infty}\right)\right)
$$

On the other hand, taking conditional expectation (i.e. an optional projection) yields

$$
I(\varepsilon)=\mathbb{E}\left(\varepsilon^{-2} \int_{0}^{\infty} d s \int_{0}^{\infty} d t f\left(\sigma_{s}^{(1)}, \sigma_{t}^{(2)}\right) Y_{s, t}\right),
$$

with $Y_{s, t}=\mathbb{P}\left(\gamma^{(1)} \in(s, s+\varepsilon), \gamma^{(2)} \in(t, t+\varepsilon) \mid \mathcal{F}_{s}^{(1)} \otimes \mathcal{F}_{t}^{(2)}\right)$. An application of the Markov property shows that

$$
Y_{s, t}=\psi_{\varepsilon}\left(\sigma_{s}^{(1)}-\sigma_{t}^{(2)}\right)
$$

where $\psi_{\varepsilon}(y)$ denotes the probability that the random sets

$$
\left\{v>0: \sigma_{v}^{(1)}+y \in \mathcal{R}^{(2)}\right\} \quad \text { and } \quad\left\{v \geq 0: \sigma_{v}^{(2)}-y \in \mathcal{R}^{(1)}\right\}
$$


are both non-empty and contained into $(0, \varepsilon)$. We thus have

$$
\begin{aligned}
I(\varepsilon) & =\mathbb{E}\left(\varepsilon^{-2} \int_{0}^{\infty} d s \int_{0}^{\infty} d t f\left(\sigma_{s}^{(1)}, \sigma_{t}^{(2)}\right) \psi_{\varepsilon}\left(\sigma_{s}^{(1)}-\sigma_{t}^{(2)}\right)\right) \\
& =\int_{0}^{\infty} \int_{0}^{\infty} f(y, z) u^{(1)}(y) u^{(2)}(z) \varepsilon^{-2} \psi_{\varepsilon}(y-z) d y d z
\end{aligned}
$$

Next, take the function $f$ in the form

$$
f(y, z)=\frac{h(y-z)}{u^{(1)}(y) u^{(2)}(z)} \varphi(y) \varphi(z)
$$

where $h:(-\infty, \infty) \rightarrow[0, \infty)$ is a continuous bounded function and $\varphi$ a continuous function with compact support included into $(0, \infty)$. We deduce from (5.8) and (5.9) that the measure $\varepsilon^{-2} \psi_{\varepsilon}(x) d x$ converges weakly as $\varepsilon \rightarrow 0+$ towards $c \delta_{0}$ for some $c>0$. Finally take $f$ in the form $f(y, z)=f(z)$ to get

$$
\mathbb{P}\left(g_{\infty} \in d t\right)=c u^{(1)}(t) u^{(2)}(t) d t .
$$

The comparison with Lemma 1.10 entails that the renewal measure $U(d t)$ of $\mathcal{R}$ is absolutely continuous with a density proportional to $u^{(1)} u^{(2)}$.

Proposition 5.9 is thus proven when $\mathcal{R}^{(1)}$ is bounded. The case when $\mathcal{R}^{(1)}$ is unbounded follows by approximation, introducing a small killing rate in $\sigma^{(1)}$.

To apply Proposition 5.9, it is crucial to know whether $\mathcal{R}^{(1)} \cap \mathcal{R}^{(2)}=\{0\}$ a.s. Because a renewal measure is a Radon measure on $[0, \infty)$, Proposition 5.9 entails that if $\mathcal{R}^{(1)}$ and $\mathcal{R}^{(2)}$ both possess renewal densities $u^{(1)}$ and $u^{(2)}$ which are continuous and positive on $(0, \infty)$, then

$$
\int_{0+} u^{(1)}(x) u^{(2)}(x) d x=\infty \Longrightarrow \mathcal{R}^{(1)} \cap \mathcal{R}^{(2)}=\{0\} \quad \text { a.s. }
$$

By a recent result in [16], the necessary and sufficient condition for $\mathcal{R}^{(1)} \cap \mathcal{R}^{(2)}=\{0\}$ a.s. is that the convolution $u^{(1)} \star u^{(2)}$ is unbounded. I know no examples in which $u^{(1)} \star u^{(2)}$ is unbounded and $\int_{0+} u^{(1)}(x) u^{(2)}(x) d x<\infty$. See also Evans [51], Rogers [135] and Fitzsimmons and Salisbury [56] for results in that direction.

The problem of characterizing the distribution of the intersection of two independent regenerative sets in the general case seems still open. We refer to [16] for the most recent results, and to Hawkes [71], Fitzsimmons et al. [54], Fristedt [60] and Molchanov [120] other works this topic. See also [14] for another geometric problem on regenerative sets involving the notion of embedding, which is connected to the preceding. 


\section{Chapter 6}

\section{Burgers equation with Brownian initial velocity}

This chapter is adapted from [15]; its purpose is to point out an interesting connection between the inviscid Burgers equation with Brownian initial velocity and certain subordinators. Applications to statistical properties of the solution are discussed.

\subsection{Burgers equation and the Hopf-Cole solution}

Burgers equation with viscosity parameter $\varepsilon>0$

$$
\partial_{t} u+\partial_{x}\left(u^{2} / 2\right)=\varepsilon \partial_{x x}^{2} u
$$

has been introduced by Burgers as a model of hydrodynamic turbulence, where the solution $u_{\varepsilon}(x, t)$ is meant to describe the velocity of a fluid particle located at $x$ at time $t$. Although it is now known that this is not a good model for turbulence, it still is widely used in physical problems as a simplified version of more elaborate models (e.g. the Navier-Stokes equation). A most important feature of (6.1) is that it is one of the very few non-linear equations that can be solved explicitly. Specifically, Hopf [75] and Cole [37] observed that applying the transformation $\gamma=2 \varepsilon \log g$ to the potential function $\gamma$ given by $\partial_{x} \gamma=-u_{\varepsilon}$, yields the heat equation $\partial_{t} g=\varepsilon \partial_{x x}^{2} g$. This enables one to determine $g$ and hence $u_{\varepsilon}$.

The asymptotic behaviour of the solution $u_{\varepsilon}$ of (6.1) as $\varepsilon$ tends to 0 is an interesting question. Roughly, $u_{\varepsilon}$ converges to a certain function $u_{0}=u$, which provides a (weak) solution of the inviscid limit equation

$$
\partial_{t} u+\partial_{x}\left(u^{2} / 2\right)=0
$$

More precisely, $u$ can be expressed implicitly in terms of the initial velocity $u(\cdot, 0)$ as follows (cf. Hopf [75], and also [142] and [140] for a brief account). Under simple conditions such as $u(\cdot, 0)=0$ on $(-\infty, 0)$ and $\liminf _{x \rightarrow \infty} u(x, 0) / x \geq 0$, the function

$$
s \rightarrow \int_{0}^{s}(t u(r, 0)+r-x) d r
$$


tends to $\infty$ as $s \rightarrow \infty$, for every $x \geq 0$ and $t>0$. We then denote by $a(x, t)$ the largest location of the overall minimum of (6.3). The mapping $x \rightarrow a(x, t)$ is right-continuous increasing; it is known as the inverse Lagrangian function. The Hopf-Cole solution to (6.2) is given by

$$
u(x, t)=\frac{x-a(x, t)}{t} .
$$

\subsection{Brownian initial velocity}

Sinai [140] and She et al. [142] have considered the inviscid Burgers equation when the initial velocity is given by a Brownian motion; see also Carraro-Duchon [33] where (6.2) is understood in some weak statistical sense. More precisely

$$
u(\cdot, 0)=0 \text { on }(-\infty, 0] \text {, and }(u(x, 0), x \geq 0) \quad \text { is a Brownian motion }
$$

is enforced from now on. Our main purpose is to point out that for each fixed $t>0$, the inverse Lagrangian function is then a subordinator; here is the precise statement.

Theorem 6.1 For each fixed $t>0$, the process $(a(x, t): x \geq 0)$ is a subordinator started from $a(0, t)$. Its Laplace exponent $\Phi$ is given by

$$
\Phi(q)=\frac{\sqrt{2 t^{2} q+1}-1}{t^{2}} .
$$

In other words, $(a(x, t)-a(0, t): x \geq 0)$ has the same distribution as the first passage process of a Brownian motion with variance $t^{2}$ and unit drift.

One can prove that the random variable $a(0, t)$ has a gamma distribution, which completes the description of the law of the inverse Lagrangian function. As this is not relevant to the applications we have in mind, we omit the proof and refer to [15] for an argument (see also Lachal [105] for the law of further variables related to $a(0, t)$ ).

Theorem 6.1 has several interesting consequences; we now briefly present a few, and refer to [18] for some further applications connected to the multifractal spectrum of the solution (see also Jaffard [86]).

The discontinuities of the Eulerian velocity $u$ are a major object of interest. Call $x>0$ an Eulerian regular point if $u$ is continuous at $x$, and an Eulerian shock point otherwise. In the latter case the amplitude of the jump $u(x, t)-u(x-, t)$ is necessarily negative (see (6.4) and Theorem 6.1); from the viewpoint of hydrodynamic turbulence, it corresponds to the velocity of the fluid particle absorbed into the shock. For each fixed $t>0$, let us write

$$
\Delta(t)=(a(x, t)-a(x-, t), x \geq 0)
$$

for the process of the jumps of the inverse Lagrangian function taken at time $t$, and recall from (6.4) that $u(x, t)-u(x-, t)=-\frac{1}{t} \Delta_{x}(t)$.

Proposition 1.3 and the Lévy-Khintchine formula

$$
\sqrt{2 q+1}-1=\frac{1}{\sqrt{2 \pi}} \int_{0}^{\infty}\left(1-\mathrm{e}^{-q y}\right) y^{-3 / 2} \exp \{-y / 2\} d y
$$

yield the following statistical description of the shocks. 
Corollary 6.2 For each fixed $t>0, \Delta(t)$ is a Poisson point process valued in $(0, \infty)$ with characteristic measure

$$
\frac{1}{t \sqrt{2 \pi y^{3}}} \exp \left\{-\frac{y}{2 t^{2}}\right\} d y \quad(y>0)
$$

Next, we turn our attention to the fractal properties of the so-called Lagrangian regular points, that are the points $y \geq 0$ for which there exists some $x \geq 0$ such that the function (6.3) reaches its overall minimum at $y=a(x, t)$ and nowhere else. A moment of reflection shows that the set $\mathcal{R}_{c}$ of Lagrangian regular points can be viewed as the range of the inverse Lagrangian function on its continuity set, i.e.

$$
\mathcal{R}_{c}=\{a(x, t): x \geq 0 \text { regular Eulerian point }\} .
$$

As $\mathcal{R}_{c}$ only differs from the range of $a(\cdot, t)$ by at most countably many points, we thus obtain as an immediate application of section 5.1 the following.

Corollary 6.3 The Hausdorff dimension and the packing dimension of $\mathcal{R}_{c}$ both equal $1 / 2$ a.s.

That the Hausdorff dimension of $\mathcal{R}_{c}$ is $1 / 2$ was the main result of Sinai [140]; see also Aspandiiarov and Le Gall [1].

Finally, we mention that Theorems 4.1 and 4.5 respectively yield the law of the iterated logarithm and the modulus of continuity of the Lagrangian function $a \rightarrow$ $x(a, t)$, that is the inverse of the function $x \rightarrow a(x, t)$; the precise statements are left to the reader. The relevance of the Lagrangian function in hydrodynamic turbulence stems from the fact that it can be viewed as the position at time $t$ of the fluid particle started from the location $a$. This can be seen from the identity $\partial_{t} x(a, t)=u(x(a, t), t)$ that follows easily from (6.4) and (6.2).

\subsection{Proof of the theorem}

Let $\Omega$ denote the set of càdlàg paths $\omega:[0, \infty) \rightarrow \mathbb{R} \cup\{\infty\}$ such that $\lim _{s \rightarrow \infty} \omega(s)=\infty$; we write $X_{s}: \omega \rightarrow \omega(s)$ for the canonical projections. Consider also the shift operators $\left(\theta_{s}: s \geq 0\right)$ and the killing operators $\left(\mathrm{k}_{s}: s \geq 0\right)$

$$
X_{r} \circ \theta_{s}=X_{r+s} \quad, \quad X_{r} \circ \mathrm{k}_{s}=\left\{\begin{array}{cc}
X_{r} & \text { if } r<s \\
\infty & \text { otherwise }
\end{array}\right.
$$

For every $x \in \mathbb{R}$, let $\mathbb{P}^{x}$ stand for the law of the Brownian motion with variance $t^{2}$ and unit drift started at $x$, which is viewed as a probability measure on $\Omega$. We next introduce the indefinite integral of $X$

$$
I_{s}=\int_{0}^{s} X_{r} d r, \quad s \geq 0
$$

its past-minimum function

$$
m_{s}=\min _{0 \leq r \leq s} I_{r}, \quad s \geq 0
$$


and the largest location of the overall minimum of $I$

$$
a=\max \left\{s \geq 0: I_{s}=m_{\infty}\right\} .
$$

Plainly, $a$ is not a stopping time. Nonetheless, there is a Markov type property at $a$ which is a special case of the so-called the Markov property at last passage times, and this provides the key to the proof of Theorem 6.1.

Lemma 6.4 For every $x \geq 0$, the processes $X \circ \mathrm{k}_{a}$ and $X \circ \theta_{a}$ are independent under $\mathbb{P}^{-x}$, and the law of $X \circ \theta_{a}$ does not depend on $x$.

Proof: The proof is based on the fact that, loosely speaking, splitting the path of a Markov process at its last passage time at a given point produces two independent processes; and more precisely, the law of the part after the last passage time does not depend of the initial distribution of the Markov process. We refer to [44] on pages 299-300 and the related references quoted therein for a precise and much more general statement.

Consider the integral process reflected at its past minimum, $I-m$. The additive property of the integral $I_{s+r}=I_{s}+I_{r} \circ \theta_{s}$ and the strong Markov property of Brownian motion readily entail that the pair $(X, I-m)$ is a strong Markov process; see the proof of Proposition VI.1 in [11] for a closely related argument. On the other hand, it should be clear that for every $x \geq 0$, we have $a<\infty$ and $X_{a}=0, \mathbb{P}^{-x}$-a.s. In particular $a$ can be viewed as the last passage time of $(X, I-m)$ at $(0,0)$, and it now follows from the aforementioned Markov property at last-passage times that the processes $(X, I-m) \circ \mathrm{k}_{a}$ and $(X, I-m) \circ \theta_{a}$ are independent and that the law of the latter does not depend on $x$. This establishes our claim.

We are now able to prove Theorem 6.1.

Proof: Fix $x \geq 0$ and $t>0$. We know from (6.5) that $(t u(s, 0)+s-x: s \geq 0)$ is a Brownian motion with variance $t^{2}$ and unit drift started at $-x$; it has the law of $X=$ $\left(X_{s}: s \geq 0\right)$ under $\mathbb{P}^{-x}$. In this framework, we can make the following identifications: The function (6.3) coincides with the integral $s \rightarrow I_{s}$, and the inverse Lagrangian function evaluated at $x$ is simply $a(x, t)=a$. Moreover, it is readily seen that for every $0 \leq z \leq x, a(z, t)$ only depends on the killed path $X \circ \mathrm{k}_{a}$.

Write $X^{\prime}=X \circ \theta_{a}, I_{s}^{\prime}=\int_{0}^{s} X_{r}^{\prime} d r$, and for $y \geq 0, a^{\prime}(y, t)$ for the largest location of the overall minimum of $s \rightarrow I_{s}^{\prime}-y s$. We then observe the identity

$$
a(x+y, t)-a=a^{\prime}(y, t) .
$$

More precisely, $a(x+y, t)$ is the largest location of the overall minimum of $s \rightarrow I_{s}-s y$. This location is bounded from below by $a(x, t)=a$, so that $a(x+y, t)-a$ is the largest location of the overall minimum of $s \rightarrow I_{a+s}-(a+s) y$. Because $I_{a+s}=I_{a}+I_{s}^{\prime}$, (6.6) follows.

According to Lemma 6.4, $X^{\prime}$ and $X \circ \mathrm{k}_{a}$ are independent. We deduce from (6.6) that the increment $a(x+y, t)-a(x, t)$ is independent of $(a(z, t): 0 \leq z \leq x)$. Because the law of $X^{\prime}$ does not depend on $x$, the same holds for $a^{\prime}(y, t)=a(x+y, t)-a(x, t)$. 
We have thus proven the independence and homogeneity of the increments of the inverse Lagrangian function.

Next, introduce $T=\min \left\{s \geq 0: X_{s}=0\right\}$, the first hitting time of 0 by $X$. By the strong Markov property, $\tilde{X}=X \circ \theta_{T}$ is independent of $X \circ \mathrm{k}_{T}$ and has the law $\mathbb{P}^{0}$. The very same argument as above shows that

$$
a(x, t)=T+\tilde{a}(0, t)
$$

where $\tilde{a}(0, t)$ stands for the largest location of the minimum of $s \rightarrow \tilde{I}_{s}=\int_{0}^{s} \tilde{X}_{r} d r$. Because $\tilde{a}(0, t)$ is independent of $T$ and has the same law as $a(0, t)$, the decompositions $a(x, t)=(a(x, t)-a(0, t))+a(0, t)$ and $(6.7)$, and the independence of the increments property show that $T$ and $a(x, t)-a(0, t)$ have the same law. In other words, the process $(a(x, t)-a(0, t): x \geq 0)$ has the same one-dimensional distributions as the first passage process $\left(T_{x}: x \geq 0\right)$ of a Brownian motion with variance $t^{2}$ and unit drift started at zero. Because both have independent and homogeneous increments, we conclude that these two processes have the same law.

Finally, the assertion that the Laplace exponent of the first passage process of a Brownian motion with variance $t^{2}$ and unit drift is given by $\Phi(q)=t^{-2}\left(\sqrt{2 t^{2} q+1}-1\right)$ is well-known; see e.g. Formula 2.0.1 on page 223 in Borodin and Salmimen [26]. 


\section{Chapter 7}

\section{Random covering}

We consider the closed subset $\mathcal{R}$ of the nonnegative half-line left uncovered by a family of random open intervals formed from a Poisson point process. This set is regenerative; one can express its Laplace exponent in terms of the characteristic measure of the Poisson point process. This enables us to determine the cases when $\mathcal{R}$ is degenerate, or bounded, or light, and also to specify its fractal dimensions. The approach relies on the correspondence between regenerative sets and subordinators.

\section{1 $\quad$ Setting}

Consider a Poisson point process $\ell=\left(\ell_{t}, t \geq 0\right)$ taking values in the positive half-line $(0, \infty)$; let $\mu$ denote its characteristic measure. This means that if $\left(\mathcal{M}_{t}\right)_{t \geq 0}$ stands for the completed natural filtration generated by $\ell$, then for every Borel set $B \subseteq[0, \infty)$, the counting process $\operatorname{Card}\left\{0 \leq s \leq t: \ell_{s} \in B\right\}, t \geq 0$, is an $\left(\mathcal{M}_{t}\right)$-Poisson process with intensity $\mu(B)$. Recall that this implies that to disjoint Borel sets correspond independent Poisson processes.

We associate to each $t \geq 0$ the open interval $I_{t}=\left(t, t+\ell_{t}\right)$ (of course, there are only a countable numbers of times when $\ell_{t} \in(0, \infty)$, so there are countably many non-empty intervals). We then consider the closed set of points in $[0, \infty)$ which are left uncovered by these random intervals:

$$
\mathcal{R}=[0, \infty)-\bigcup_{t \geq 0} I_{t}
$$

For short, we will refer to $\mathcal{R}$ as the uncovered set in the sequel. If $\mu((\varepsilon, \infty))=\infty$ for some $\varepsilon>0$, then the set $\left\{t: \ell_{t}>\varepsilon\right\}$ is everywhere dense a.s., and it follows that $\mathcal{R}=$ $\{0\}$ a.s. This trivial case is henceforth excluded, and we denote by $\bar{\mu}(x)=\mu((x, \infty))$, $x>0$, the tail of $\mu$.

The problem of finding a necessary and sufficient condition for $\mathcal{R}$ to reduce to $\{0\}$, was raised by Mandelbrot [113] and solved by Shepp [143]. Previously, Dvoretzky asked a closely related question on covering the circle with random arcs; see chapter 11 in Kahane [91] for further references on this topic. To tackle this question, we will follow a method due to Fitzsimmons, Fristedt and Shepp [55], which also enables us 
to settle many other natural questions about $\mathcal{R}$. The approach relies on the following intuitively obvious observation:

Lemma 7.1 If 0 is not isolated in $\mathcal{R}$ a.s., then $\mathcal{R}$ is a perfect regenerative set.

Proof: We first verify that the uncovered set is progressively measurable. Take any $0<s<t$ and note that

$$
[s, t] \subseteq \bigcup_{v \geq 0} I_{v} \Longleftrightarrow[s, t] \subseteq \bigcup_{0 \leq v \leq t, \ell_{v}>1 / n} I_{v} \quad \text { for some large enough } n
$$

Indeed, the interval $I_{v}$ does not intersect $[s, t]$ for $v \geq t$; and from any cover of $[s, t]$ by a family of open intervals, we can extract a cover by a finite sub-family. Next, fix an integer $n$. The Poisson point process $\ell$ restricted to $(1 / n, \infty)$ is discrete (since $\bar{\mu}(1 / n)<\infty)$; and it can be easily deduced that the event

$$
\left\{[s, t] \text { is covered by }\left(I_{v}: \ell_{v}>1 / n \text { and } 0 \leq v \leq t\right)\right\}
$$

is $\mathcal{M}_{t^{-}}$measurable. Hence, the event $\left\{[s, t]\right.$ is covered by $\left.\left(I_{v}, v \geq 0\right)\right\}$ is also $\mathcal{M}_{t^{-}}$ measurable. Writing $G_{t}=g_{t+}=\sup \{u \leq t: u \in \mathcal{R}\}$, the equivalence

$$
G_{t}<s \Longleftrightarrow[s, t] \subseteq \bigcup_{v \geq 0} I_{v}
$$

shows that the right-continuous process $\left(G_{t}: t \geq 0\right)$ is adapted, and thus optional. It follows that $\mathcal{R}=\left\{t: t-G_{t}=0\right\}$ is progressively measurable.

We next check that $\mathcal{R}$ has no isolated points a.s. For any fixed $t>0$, it is easily seen that $D_{t-}=\inf \{s \geq t: s \in \mathcal{R}\}$ is an announceable stopping time ${ }^{1}$. It is well known that a Poisson point process does not jump at an announceable stopping time, so the shifted point process $\ell^{\prime}=\left(\ell_{D_{t-}+s}, s \geq 0\right)$ is again a Poisson point process with intensity $\mu$. Since the collection of intervals $\left(I_{v}: 0 \leq v<D_{t-}\right)$ do not cover $D_{t-}$, they do not cover any $s>D_{t-}$ either. In other words, $s>D_{t-}$ is covered by the intervals $\left(I_{v}: v \geq 0\right)$ if and only $s-D_{t-}$ is covered by $\left(\left(v, v+\ell_{v}^{\prime}\right): v \geq 0\right)$. We know by assumption that 0 is not isolated in $\mathcal{R}$ a.s., and this implies that $D_{t-}$ is not isolated in $\mathcal{R}$ either. Any positive instant in $\mathcal{R}$ which is isolated on its left can be expressed in the form $D_{t-}$ for some rational number $t>0$. We conclude that $\mathcal{R}$ has no isolated points a.s.

Finally, we establish the regenerative property. Let $T$ be an arbitrary $\left(\mathcal{M}_{t}\right)$ stopping time, which is a right-accumulation point of $\mathcal{R}$ a.s. on $\{T<\infty\}$. Then $T$ is not a jump time of $\ell$, for if it were, then $I_{T}$ would be a right-neighbourhood of $T$. As a consequence, conditionally on $\{T<\infty\}$, the shifted point process $\ell^{\prime}=\left(\ell_{T+t}, t \geq 0\right)$ is independent of $\mathcal{M}_{T}$ and is again a Poisson point process with intensity $\mu$. By the same argument as in the preceding paragraph, an instant $s>T$ is covered by the intervals $\left(I_{t}: t \geq 0\right)$, if and only $s-T$ is covered by $\left(\left(t, t+\ell_{t}^{\prime}\right): t \geq 0\right)$. This shows that $\mathcal{R}$ is regenerative.

\footnotetext{
${ }^{1}$ Specifically, consider the process $X_{u}=\sup \left\{s+\ell_{s}-u, 0 \leq s<u\right\}, u \geq 0$; note that $X$ is adapted with càdlàg paths and no negative jumps. In this setting $D_{t-}$ coincides with the limit of the increasing sequence of stopping times $\inf \left\{s \geq q: X_{s} \leq 1 / n\right\}, n=1,2, \cdots$.
} 


\subsection{The Laplace exponent of the uncovered set}

Lemma 7.1 enables us to identify the uncovered set as the range of some subordinator $\sigma$, whenever 0 is not isolated in $\mathcal{R}$. This will allow us to derive information on $\mathcal{R}$ from known results of subordinators, if we are able to characterize $\sigma$ in terms of the characteristic measure $\mu$ of the Poisson point process. This motivates the main result of this section, which provides an explicit formula for the Laplace exponent $\Phi$ of $\sigma$. Recall that $\bar{\mu}$ denotes the tail of $\mu$.

Theorem 7.2 (Fitzsimmons, Fristedt and Shepp [55]) If

$$
\int_{0}^{1} \exp \left\{\int_{t}^{1} \bar{\mu}(s) d s\right\} d t=\infty
$$

then $\mathcal{R}=\{0\}$ a.s. Otherwise, $\mathcal{R}$ is a perfect regenerative set, and the Laplace exponent of the corresponding subordinator is given by

$$
\frac{1}{\Phi(\lambda)}=c \int_{0}^{\infty} \mathrm{e}^{-\lambda t} \exp \left\{\int_{t}^{1} \bar{\mu}(s) d s\right\} d t, \quad \lambda>0
$$

where $c>0$ is the constant of normalization (recall that $\Phi(1)=1$ ).

Using the fact that the Laplace transform of the renewal measure is $1 / \Phi$, one can rephrase the statement as follows: When the uncovered set is not trivial, the renewal measure is absolutely continuous with density

$$
u(t)=c \exp \left\{\int_{t}^{1} \bar{\mu}(s) d s\right\}, \quad t \geq 0 .
$$

For instance, when the tail of the characteristic measure is $\bar{\mu}(x)=\beta x^{-1}$ for some $\beta>0$, then $\exp \left\{\int_{t}^{1} \bar{\mu}(s) d s\right\}=t^{-\beta}$. We get from Theorem 7.2 that $\mathcal{R}$ reduces to $\{0\}$ a.s. if $\beta \geq 1$, and otherwise $\Phi(\lambda)=\lambda^{1-\beta}$, that is $\mathcal{R}$ is the range of a stable subordinator of index $1-\beta$.

Proof: We will prove the theorem first in the simple case when the Poisson point process is discrete, and then deduce the general case by approximation. So we first suppose that $\bar{\mu}(0+)<\infty$; in particular the integral in Theorem 7.2 converges. Then $\ell$ is a discrete Poisson point process and $\mathcal{R}$ plainly contains some right-neighbourhood of the origin. A fortiori 0 is not isolated in $\mathcal{R}$ a.s., and by Lemma 7.1, $\mathcal{R}$ is a heavy regenerative set.

A fixed time $t>0$ is uncovered if and only if $\ell_{s} \leq t-s$ for every $s<t$; which entails that

$$
\mathbb{P}(t \in \mathcal{R})=\exp \left\{-\int_{0}^{t} \bar{\mu}(t-s) d s\right\}>0 .
$$

It then follows from Proposition 1.9(ii) that the renewal density of $\mathcal{R}$ at $t$ is proportional to $\exp \left\{-\int_{0}^{t} \bar{\mu}(t-s) d s\right\}$, which is the same as (7.1), and this proves the theorem in the discrete case.

We then deduce the general case when $\bar{\mu}(0+)=\infty$ by approximation. For every integer $n>0$, let $\ell^{(n)}=\left(\ell_{t}: t \geq 0\right.$ and $\left.\ell_{t}>1 / n\right)$ denote the discrete Poisson point 
process restricted to $(1 / n, \infty)$, and $\mathcal{R}^{(n)}$ the corresponding uncovered set. We know that the Laplace exponent associated with $\mathcal{R}^{(n)}$ is given by

$$
\frac{1}{\Phi^{(n)}(\lambda)}=c_{n} \int_{0}^{\infty} \mathrm{e}^{-\lambda t} \exp \left\{\int_{t}^{1} \bar{\mu}\left(s \vee n^{-1}\right) d s\right\} d t .
$$

For every $s>0, \bar{\mu}\left(s \vee n^{-1}\right)$ increases to $\bar{\mu}(s)$ as $n \rightarrow \infty$. It follows that the probability measure on $[0, \infty)$,

$$
c_{n} \mathrm{e}^{-t} \exp \left\{\int_{t}^{1} \bar{\mu}\left(s \vee n^{-1}\right) d s\right\} d t
$$

converges in the weak sense towards

$$
c_{\infty} \mathrm{e}^{-t} \exp \left\{\int_{t}^{1} \bar{\mu}(s) d s\right\} d t
$$

(where $c_{\infty}$ is the normalizing constant) if $\int_{0}^{1} \exp \left\{\int_{t}^{1} \bar{\mu}(s) d s\right\} d t<\infty$, and towards the Dirac point mass at 0 otherwise. Considering Laplace transforms, we deduce that for every $\lambda>0, \lim _{n \rightarrow \infty} \Phi^{(n)}(\lambda)=\Phi^{(\infty)}(\lambda)$, where

$$
\frac{1}{\Phi^{(\infty)}(\lambda)}=\left\{\begin{array}{cc}
1 & \text { if } \int_{0}^{1} \exp \left\{\int_{t}^{1} \bar{\mu}(s) d s\right\} d t=\infty, \\
c_{\infty} \int_{0}^{\infty} \mathrm{e}^{-\lambda t} \exp \left\{\int_{t}^{1} \bar{\mu}(s) d s\right\} d t & \text { otherwise. }
\end{array}\right.
$$

On the other hand, $\left(\mathcal{R}^{(n)}: n \in \mathbb{N}\right)$ is a decreasing sequence of random closed sets and $\mathcal{R}=\bigcap \mathcal{R}^{(n)}$. As a consequence, we have

$$
G_{t}^{(n)}=\sup \left\{s \leq t: s \in \mathcal{R}^{(n)}\right\} \longrightarrow \sup \{s \leq t: s \in \mathcal{R}\}=G_{t} \quad(\text { as } n \rightarrow \infty) .
$$

We deduce from Lemma 1.11 that for every $\lambda>0$

$$
\int_{0}^{\infty} \mathrm{e}^{-t} \mathbb{E}\left(\exp \left\{-\lambda G_{t}\right\}\right) d t=\frac{1}{\Phi^{(\infty)}(\lambda+1)} .
$$

Suppose first that $\int_{0}^{1} \exp \left\{\int_{t}^{1} \bar{\mu}(s) d s\right\} d t<\infty$. We see from $(7.2)$ that $\Phi^{(\infty)}(\lambda)$ goes to $\infty$ as $\lambda \rightarrow \infty$. Together with (7.3), this forces $\mathbb{P}\left(G_{t}=0\right)=0$ for almost every $t \geq 0$; which means that 0 is not isolated in $\mathcal{R}$. We then know from Lemma 7.1 that $\mathcal{R}$ is regenerative; comparing (7.3) and Lemma 1.11 shows that its Laplace exponent must be $\Phi=\Phi^{(\infty)}$.

Finally, suppose that $\int_{0}^{1} \exp \left\{\int_{t}^{1} \bar{\mu}(s) d s\right\} d t=\infty$, so $\lim _{n \rightarrow \infty} \Phi^{(n)}(\lambda)=1$ for every $\lambda>0$. We deduce from (7.3) that $\mathbb{P}\left(G_{t}=0\right)=1$ for almost every $t \geq 0$, that is $\mathcal{R}=\{0\}$ a.s.

\subsection{Some properties of the uncovered set}

We suppose throughout this subsection that

$$
\int_{0}^{1} \exp \left\{\int_{t}^{1} \bar{\mu}(s) d s\right\} d t<\infty,
$$

that is that $\mathcal{R}$ is not degenerate to the single point $\{0\}$, a.s. We immediately get the following features. 
Corollary 7.3 $\mathcal{R}$ is heavy or light according as the integral $\int_{0}^{1} \bar{\mu}(t) d t$ converges or diverges.

Proof: We know from Proposition 1.8 that a regenerative set is heavy or light according as the drift coefficient $d$ is zero or positive. On the other hand, recall that

$$
\mathrm{d}=\lim _{\lambda \rightarrow \infty} \lambda^{-1} \Phi(\lambda)
$$

According to Theorem 7.2, we have by an integration by parts

$$
\frac{\lambda}{\Phi(\lambda)}=c \int_{0}^{\infty}\left(1-\mathrm{e}^{-\lambda t}\right) \bar{\mu}(t) \exp \left\{\int_{t}^{1} \bar{\mu}(s) d s\right\} d t, \quad \lambda>0
$$

and we deduce by monotone convergence that

$$
\frac{1}{\mathrm{~d}}=c \int_{0}^{\infty} \bar{\mu}(t) \exp \left\{\int_{t}^{1} \bar{\mu}(s) d s\right\} d t=c \exp \left\{\int_{0}^{1} \bar{\mu}(s) d s\right\}-c \exp \left\{-\int_{1}^{\infty} \bar{\mu}(s) d s\right\} .
$$

We conclude that $\mathrm{d}=0$ iff $\int_{0}^{1} \bar{\mu}(s) d s=\infty$.

Alternatively, one may also deduce the result from Proposition 1.9 and the easy fact that the probability that the point 1 is left uncovered equals $\exp \left\{-\int_{0}^{1} \bar{\mu}(s) d s\right\}$.

Corollary 7.4 If $\int_{1}^{\infty} \exp \left\{-\int_{1}^{t} \bar{\mu}(s) d s\right\} d t=\infty$, then $\mathcal{R}$ is unbounded a.s. Otherwise, $\mathcal{R}$ is bounded a.s. and the distribution of the largest uncovered point

$$
g_{\infty}=\sup \{s \geq 0: s \in \mathcal{R}\}
$$

is given by

$$
\mathbb{P}\left(g_{\infty} \in d t\right)=k^{-1} \exp \left\{\int_{t}^{1} \bar{\mu}(s) d s\right\} d t, \quad \text { with } k=\int_{0}^{\infty} \exp \left\{\int_{t}^{1} \bar{\mu}(s) d s\right\} d t .
$$

Proof: According to (2.2), the probability that $\mathcal{R}$ is bounded equals 0 or 1 according as the killing rate $\mathrm{k}=\Phi(0)$ is zero or positive. It follows immediately from Theorem 7.2 that

$$
\mathrm{k}=0 \Longleftrightarrow \int_{1}^{\infty} \exp \left\{-\int_{1}^{t} \bar{\mu}(s) d s\right\}=\infty
$$

When $\mathcal{R} \neq\{0\}$ is bounded a.s., the formula for the distribution of $g_{\infty}$ follows from Lemma 1.11 and the expression (7.1) for the density of the renewal measure.

Motivated by the limit theorem 3.2 for the process of the last passage times in $\mathcal{R}$, we next investigate the asymptotic behaviour of the Laplace exponent $\Phi$.

Corollary 7.5 For every $\alpha \in(0,1]$, the following assertions are equivalent:

(i) $\bar{\mu}(s) \sim(1-\alpha) s^{-1}$ as $s \rightarrow \infty$ (for $\alpha=1$, this means that $\bar{\mu}(s)=o\left(s^{-1}\right)$ ).

(ii) $\Phi$ is regularly varying at $0+$ with index $\alpha$. 
Proof: Recall from Proposition 1.5 that $\Phi$ is regularly varying at $0+$ with index $\alpha$ if and only if the renewal function $U$ is regularly varying at $\infty$ with index $\alpha$. We know from (7.1) that $U$ has a decreasing derivative $u$, so the monotone density theorem applies and (ii) holds if and only if $u$ is regularly varying at $\infty$ with index $\alpha-1$ (cf. [20] on page 39).

Using again (7.1), we have

$$
t^{1-\alpha} u(t)=c \exp \left\{\int_{1}^{t}\left((1-\alpha) s^{-1}-\bar{\mu}(s)\right) d s\right\}
$$

and it is then plain from the theorem of representation of slowly varying functions (cf. [20] on page 12) that (i) implies that $u$ is regularly varying at $\infty$ with index $\alpha-1$. Conversely, suppose that $u$ is regularly varying at $\infty$ with index $\alpha-1$, so that by the theorem of representation of slowly varying functions

$$
\int_{1}^{t}\left((1-\alpha) s^{-1}-\bar{\mu}(s)\right) d s=c(t)+\int_{1}^{t} \varepsilon(s) s^{-1} d s,
$$

where $\lim _{t \rightarrow \infty} c(t) \in \mathbb{R}$ and $\lim _{t \rightarrow \infty} \varepsilon(t)=0$. It then follows readily from the monotonicity of $\bar{\mu}$ that this representation is possible only if (i) holds.

We next turn our attention to the fractal dimensions of the uncovered set, which are given by the lower and upper indices of the Laplace exponent, see Theorem 5.1.

Corollary 7.6 The lower and upper indices are given by

$$
\begin{aligned}
& \underline{\text { ind }}(\Phi)=\sup \left\{\rho: \lim _{t \rightarrow 0+} t^{1-\rho} \exp \left\{\int_{t}^{1} \bar{\mu}(s) d s\right\}=0\right\}=1-\limsup _{t \rightarrow 0+}\left(\frac{\int_{t}^{1} \bar{\mu}(s) d s}{\log 1 / t}\right), \\
& \overline{\operatorname{ind}}(\Phi)=\inf \left\{\rho: \lim _{t \rightarrow 0+} t^{1-\rho} \exp \left\{\int_{t}^{1} \bar{\mu}(s) d s\right\}=\infty\right\}=1-\liminf _{t \rightarrow 0+}\left(\frac{\int_{t}^{1} \bar{\mu}(s) d s}{\log 1 / t}\right) .
\end{aligned}
$$

Proof: For the sake of conciseness, we focus on the lower index. We get from the formula for $\Phi$ in Theorem 7.2

$$
\begin{aligned}
\underline{\text { ind }}(\Phi) & =\sup \left\{\rho: \lim _{\lambda \rightarrow \infty} \lambda^{\rho} \int_{0}^{\infty} \mathrm{e}^{-\lambda t} \exp \left\{\int_{t}^{1} \bar{\mu}(s) d s\right\} d t=0\right\} \\
& =\sup \left\{\rho: \lim _{\lambda \rightarrow \infty} \lambda^{\rho-1} \int_{0}^{\infty} \mathrm{e}^{-t} \exp \left\{\int_{t / \lambda}^{1} \bar{\mu}(s) d s\right\} d t=0\right\} .
\end{aligned}
$$

Using the immediate inequality

$$
\int_{0}^{\infty} \mathrm{e}^{-t} \exp \left\{\int_{t / \lambda}^{1} \bar{\mu}(s) d s\right\} d t \geq \mathrm{e}^{-1} \exp \left\{\int_{1 / \lambda}^{1} \bar{\mu}(s) d s\right\},
$$

we deduce

$$
\underline{\text { ind }}(\Phi) \leq \sup \left\{\rho: \lim _{t \rightarrow 0+} t^{1-\rho} \exp \left\{\int_{t}^{1} \bar{\mu}(s) d s\right\}=0\right\} .
$$

To prove the converse inequality, we may suppose that there is $\rho>0$ such that

$$
\lim _{t \rightarrow 0+} t^{1-\rho} \exp \left\{\int_{t}^{1} \bar{\mu}(s) d s\right\}=0
$$


(otherwise there is nothing to prove). Recall that the renewal measure has density $u$ given by (7.1), so that $u(t)=o\left(t^{\rho-1}\right)$ and then $U(t)=o\left(t^{\rho}\right)$ as $t \rightarrow 0+$, for every $\varepsilon>0$. Applying Proposition 1.4, this entails $\lim _{\lambda \rightarrow \infty} \lambda^{-\rho} \Phi(\lambda)=\infty$, and thus ind $(\Phi) \geq \rho$.

The identification of the uncovered set in terms of a subordinator $\sigma$ enables us to invoke results of section 3.2 to decide whether a given Borel set $B \subseteq(0, \infty)$ is completely covered by the random intervals. Typically, recall Propositions 5.6 and 5.7 which are relevant as the renewal density $u$ is a decreasing function (by (7.1)). If the Hausdorff dimension of $B$ is greater that $1-\underline{\text { ind }}(\Phi)$, then the probability that $B$ is not completely covered is positive. On the other hand, if the Hausdorff dimension of $B$ is less that $1-\overline{\operatorname{ind}}(\Phi)$, then $B$ is completely covered a.s. Of course, (5.6) provides a complete (but not quite explicit) characterization of sets which are completely covered by the random intervals.

Finally, let us mention an interesting problem raised by Pat Fitzsimmons (private communication). It is easily seen that the uncovered set $\mathcal{R}$ is an infinitely divisible regenerative set, in the sense that for every integer $n, \mathcal{R}$ can be expressed as the intersection of $n$-independent regenerative sets with the same distribution. Conversely, can any (perfect) infinitely divisible regenerative set be viewed of as a set left uncovered by random intervals sampled from a Poisson point process? Kendall [93] gave a positive answer in the heavy case. The light case seems to be still open. 


\section{Chapter 8}

\section{Lévy processes}

Real-valued Lévy processes give rise to two interesting families of regenerative sets: the set of times when a fixed point is visited, and the set of times when a new supremum is reached. Some applications are given in the special case when the Lévy process has no positive jumps. Some applications of Bochner's subordination to Lévy processes are also discussed.

\subsection{Local time at a fixed point}

Throughout this chapter, $\left(X_{t}: t \geq 0\right)$ will denote a real-valued Lévy process, i.e. $X$ has independent and homogeneous increments and càdlàg paths. For instance the difference of two independent strict subordinators is a Lévy process. For every $x \in$ $\mathbb{R}$, write $\mathbf{P}^{x}$ for the distribution of the process $X+x$; it is well-known that $X=$ $\left(\Omega, \mathcal{M}, \mathcal{M}_{t}, X_{t}, \theta_{t}, \mathbf{P}^{x}\right)$ is a Feller process (see e.g. [11], Chapter I). The purpose of this section is to study the regularity of a fixed point $r$, and then to determine the distribution of its local time. To this end, we need information on the resolvent operator $V^{q}$.

To start with, recall that the characteristic function of $X_{t}$ can be expressed in the form

$$
\mathbf{E}^{0}\left(\mathrm{e}^{\mathrm{i} \lambda X_{t}}\right)=\mathrm{e}^{-t \Psi(\lambda)}, \quad \lambda \in \mathbb{R}, t \geq 0,
$$

where $\Psi: \mathbb{R} \rightarrow \mathbb{C}$. One calls $\Psi$ the characteristic exponent of $X$; it can be expressed via the Lévy-Khintchine's formula (which is more general than that which we discussed in Section 1.2 in the special case of subordinator):

$$
\Psi(\lambda)=\mathrm{i} a \lambda+\frac{1}{2} b \lambda^{2}+\int_{\mathbb{R}}\left(1-\mathrm{e}^{\mathrm{i} \lambda x}+\mathrm{i} \lambda x \mathbf{1}_{\{|x|<1\}}\right) \Lambda(d x),
$$

where $a \in \mathbb{R}, b \geq 0$ is called the Gaussian coefficient, and $\Lambda$ a measure on $\mathbb{R}-\{0\}$ with $\int\left(1 \wedge|x|^{2}\right) \Lambda(d x)<\infty$ called the Lévy measure. It follows that for every Lebesgueintegrable function $f$ and $q>0$, we have 


$$
\begin{aligned}
\int_{-\infty}^{\infty} \mathrm{e}^{\mathrm{i} \lambda x} V^{q} f(x) d x & =\int_{-\infty}^{\infty} \mathrm{e}^{\mathrm{i} \lambda x}\left(\int_{0}^{\infty} \mathbf{E}^{x}\left(f\left(X_{t}\right)\right) \mathrm{e}^{-q t} d t\right) d x \\
& =\int_{0}^{\infty} \mathrm{e}^{-q t}\left(\int_{-\infty}^{\infty} \mathrm{e}^{\mathrm{i} \lambda x} \mathbf{E}^{0}\left(f\left(X_{t}+x\right)\right) d x\right) d t \\
& =\int_{0}^{\infty} \mathrm{e}^{-q t}\left(\int_{-\infty}^{\infty} \mathrm{e}^{\mathrm{i} \lambda y} f(y) \mathbf{E}^{0}\left(\mathrm{e}^{-\mathrm{i} \lambda X_{t}}\right) d y\right) d t \\
& =\left(\int_{0}^{\infty} \mathrm{e}^{-q t} \exp \{-t \Psi(-\lambda)\} d t\right)\left(\int_{-\infty}^{\infty} \mathrm{e}^{\mathrm{i} \lambda y} f(y) d y\right) \\
& =\frac{1}{q+\Psi(-\lambda)}\left(\int_{-\infty}^{\infty} \mathrm{e}^{\mathrm{i} \lambda y} f(y) d y\right) .
\end{aligned}
$$

In other words, if $\mathcal{F}(g)$ stands for the Fourier transform of an integrable function $g$, then

$$
\mathcal{F}\left(V^{q} f\right)(\lambda)=\frac{\mathcal{F}(f)(\lambda)}{q+\Psi(-\lambda)}
$$

We are now able to prove the following basic result which goes back to Orey [123].

Proposition $8.1{ }^{1}$ Suppose that the characteristic exponent $\Psi$ satisfies

$$
\int_{-\infty}^{\infty}|q+\Psi(\lambda)|^{-1} d \lambda<\infty
$$

for some (and then all) $q>0$. Then every point $r \in \mathbb{R}$ is regular for itself and the Laplace exponent $\Phi$ of the inverse local time is given by

$$
\frac{1}{\Phi(q)}=c \int_{-\infty}^{\infty} \frac{d \lambda}{q+\Psi(\lambda)}, \quad q>0
$$

where $c>0$ is the constant of normalization.

Proof: The function

$$
v^{q}(x)=\frac{1}{2 \pi} \int_{-\infty}^{\infty} \frac{\mathrm{e}^{-\mathrm{i} x \lambda}}{q+\Psi(\lambda)} d \lambda, \quad x \in \mathbb{R}
$$

is continuous and its Fourier transform is $\lambda \rightarrow 1 /(q+\Psi(\lambda))$. By Fourier inversion, we deduce from (8.2) that

$$
V^{q} f(x)=\int_{-\infty}^{\infty} f(y) v^{q}(y-x) d y
$$

\footnotetext{
${ }^{1}$ We mention for completeness that Bretagnolle [30] has established a sharper and much more difficult result: a necessary and sufficient condition for 0 to be regular for itself is

$$
\int_{-\infty}^{\infty} \Re\left(\frac{1}{1+\Psi(\lambda)}\right) d \lambda<\infty \text { and } X \text { has unbounded variation. }
$$
}


In other words, the $q$-resolvent operator of $X$ has a continuous density kernel $v^{q}(x, y)=$ $v^{q}(y-x)$ with respect to the Lebesgue measure. Plainly $\widehat{X}=-X$ is also a Lévy process and the very same calculations show that its $q$-resolvent operator is given by

$$
\widehat{V}^{q} f(x)=\int_{-\infty}^{\infty} f(y) v^{q}(x-y) d y .
$$

Hence, $X$ and $\widehat{X}$ are in duality with respect to the Lebesgue measure, and the condition (iii) of Proposition 2.2 is fulfilled. This yields our statement.

It is easily seen that when local times exist, they can be expressed as occupation densities, in the sense that the local time at level $r \in \mathbb{R}$ is given by $L(r, \cdot)=$ $\lim _{\varepsilon \rightarrow 0+}(2 \varepsilon)^{-1} \int_{0}^{\cdot} \mathbf{1}_{\left.\left\{\mid X_{t}-r\right] \leq \varepsilon\right\}} d t$. See section V.1 in [11] for details. A major problem in this field is to decide whether the mapping $(r, t) \rightarrow L(r, t)$ has a continuous version. This has been solved in a remarkable paper by Barlow [4], see also [3] and Marcus and Rosen [114] in the symmetric case.

Proposition 8.1 provides a simple expression for the Laplace exponent $\Phi$ of the inverse local time, which is explicit in terms of the characteristic exponent $\Psi$. This enables one to directly apply the general results proven in the preceding chapters; here is an example. Suppose for simplicity that $X$ is symmetric and that the condition of Proposition 8.1 is fulfilled. We should like to express the condition

$$
\Phi \text { is regularly varying with index } \rho \in(0,1) \quad(\text { at } 0+\text {, resp. at } \infty)
$$

in terms of $\Psi$. This question is motivated for instance by the Dynkin-Lamperti theorem 3.2. Alternatively, (8.3) has an important rôle in the law of the iterated logarithm for local times (which has been considered in particular by Marcus and Rosen $[115,116])$. The assumption of symmetry ensures that the characteristic exponent $\Psi$ is an even real-valued function. We write $\Psi^{\uparrow}$ for the so-called increasing rearrangement of $\Psi$, viz.

$$
\Psi^{\uparrow}(x)=m(\lambda \in \mathbb{R}: \Psi(\lambda) \leq x) \quad(x \geq 0)
$$

where $m$ refers to the Lebesgue measure. By Proposition 8.1, we have

$$
\begin{aligned}
\frac{1}{c \Phi(q)}=\int_{[0, \infty)} \frac{1}{q+x} d \Psi^{\uparrow}(x) & =\int_{[0, \infty)}\left(\int_{0}^{\infty} \mathrm{e}^{-(q+x) t} d t\right) d \Psi^{\uparrow}(x) \\
& =\int_{0}^{\infty} \mathrm{e}^{-q t} \mathcal{L} \Psi^{\uparrow}(t) d t
\end{aligned}
$$

where $\mathcal{L} \Psi^{\uparrow}(t)=\int_{[0, \infty)} \mathrm{e}^{-t x} d \Psi^{\uparrow}(x)$ is the Laplace transform of the measure with distribution function $\Psi^{\uparrow}$. Because $\Phi$ is regularly varying with index $\rho$ if and only if $1 / \Phi$ is regularly varying with index $-\rho$, we deduce from a tauberian theorem that (8.3) holds if and only if the indefinite integral of $\mathcal{L} \Psi^{\uparrow}, \int_{0}^{\cdot} \mathcal{L} \Psi^{\uparrow}(t) d t$, is regularly varying with index $\rho$ (at $\infty$, resp. at $0+)$. Plainly, the indefinite integral of $\mathcal{L} \Psi^{\uparrow}$ has a decreasing derivative, so by the monotone density theorem, the latter is equivalent to $\mathcal{L} \Psi^{\uparrow}$ varying regularly with index $\rho-1$ (at $\infty$, resp. at $0+$ ). We then again invoke a tauberian theorem to conclude that

$$
\Longleftrightarrow \quad \Psi^{\uparrow} \text { varies regularly with index } 1-\rho . \quad(\text { at } 0+, \text { resp. at } \infty) \text {. }
$$


More precisely, the preceding argument shows that when (8.3) holds, then

$$
\Phi(q) \sim c^{\prime} q / \Psi^{\uparrow}(q) \quad(\text { at } 0+\text {, resp. at } \infty)
$$

for some positive finite constant number $c^{\prime}$ which can be expressed explicitly in terms of our data.

\subsection{Local time at the supremum}

We next turn our attention the supremum process $S .=\sup \left\{X_{s}: 0 \leq s \leq \cdot\right\}$. It is easy to check that the so-called reflected process $S-X$ is a Feller process; see Proposition VI.1 in [11]. The closed zero set of the reflected process

$$
\mathcal{R}=\overline{\left\{t \geq 0: X_{t}=S_{t}\right\}}
$$

coincides with the set of times when the Lévy process reaches a new supremum. It is known as the ladder time set. There is a simple criterion due to Rogozin [133] to decide whether 0 is regular for itself with respect to the reflected process:

$$
\mathcal{R} \text { is perfect } \Longleftrightarrow \int_{0+} t^{-1} \mathbf{P}^{0}\left(X_{t} \geq 0\right) d t=\infty .
$$

See also [13] for an equivalent condition in terms of the Lévy measure of $X$.

We henceforth suppose that $\mathcal{R}$ is perfect; the Laplace exponent of the ladder time set can be expressed as follows:

$$
\Phi(q)=\exp \left\{\int_{0}^{\infty}\left(\mathrm{e}^{-t}-\mathrm{e}^{-q t}\right) t^{-1} \mathbf{P}^{0}\left(X_{t} \geq 0\right) d t\right\}, \quad q \geq 0 .
$$

Formula (8.4) is a special case of a result of Fristedt (see e.g. Corollary VI.10 in [11] and the comments thereafter), and goes back to Spitzer in discrete time. The main drawback of (8.4) is that it involves the probabilities $\mathbf{P}^{0}\left(X_{t} \geq 0\right)$ which are usually not known explicitly. For instance, Bingham [19] has raised the question of determining the class of Laplace exponents which can arise in connection with ladder time sets. This interesting problem seems to be still open. ${ }^{2}$

As an example of an application of (8.4) motivated by Chapter 4, we consider the question of whether the Laplace exponent of a ladder time set has the asymptotic behaviour that is required in the Dynkin-Lamperti Theorem 3.2.

Proposition 8.2 For each fixed $\alpha \in[0,1], \Phi$ is regularly varying with index $\alpha$ at $0+$ (respectively, at $\infty$ ) if and only if

$$
\left.\lim \frac{1}{t} \int_{0}^{t} \mathbf{P}^{0}\left(X_{s} \geq 0\right) d s=\alpha \quad \text { as } t \rightarrow \infty \text { (respectively, as } t \rightarrow 0+\right) .
$$

\footnotetext{
${ }^{2}$ By an application of the Frullani integral to (8.4), one sees that the function $q \rightarrow q / \Phi(q)$ must be the Laplace exponent of a subordinator; cf. the proof of Theorem 8.3 below. In particular ladder time processes form a strict sub-class of subordinators.
} 
Proof: We know from Theorem 3.2 that $\Phi$ is regularly varying with index $\alpha$ at $0+$ (respectively, at $\infty$ ) if and only if $\lim q \Phi^{\prime}(q) / \Phi(q)=\alpha$ as $q \rightarrow 0+$ (respectively, as $q \rightarrow \infty)$. According to (8.4), the logarithmic derivative of $\Phi$ is given by

$$
\frac{\Phi^{\prime}(q)}{\Phi(q)}=\int_{0}^{\infty} \mathrm{e}^{-q t} \mathbf{P}^{0}\left(X_{t} \geq 0\right) d t
$$

By a Tauberian theorem, the right-hand side is equivalent to $\alpha / q$ if and only if (8.5) holds.

One refers to (8.5) as Spitzer's condition; it has a crucial rôle in developing fluctuation theory for Lévy processes, in particular in connection with estimates for the distribution of first passage times and for the asymptotic behaviour of the time spent by the Lévy process in the positive semi-axis. See Chapter VI in [11]. It is natural to compare (8.5) with the apparently stronger condition

$$
\lim \mathbf{P}^{0}\left(X_{t} \geq 0\right)=\alpha \quad \text { as } t \rightarrow \infty \text { (respectively, as } t \rightarrow 0+\text { ). }
$$

We will refer to (8.6) as Doney's condition, for Doney [47] has recently proven that the discrete time versions of (8.5) and (8.6) are equivalent, settling a question that has puzzled probabilists for a long time. We present here the analogous result in continuous time.

Theorem 8.3 The conditions of Spitzer and Doney are equivalent.

Proof: We shall only prove the theorem for $0<\alpha<1$ and $t \rightarrow 0+$, and we refer to [17] for the complete argument. The implication $(8.6) \Rightarrow(8.5)$ is obvious, so we assume that (8.5) holds. Notice that the case when $X$ is a compound Poisson process with a possible drift is then ruled out; this ensures that $\mathbf{P}^{0}\left(X_{t}=0\right)=0$ for all $t>0$, and as a consequence, the mapping $t \rightarrow \mathbf{P}^{0}\left(X_{t} \geq 0\right)$ is continuous on $(0, \infty)$.

Introduce the Laplace exponent $\widehat{\Phi}$ of the dual ladder time set which corresponds to the Lévy process $\widehat{X}=-X$. This means

$$
\begin{aligned}
\widehat{\Phi}(q) & =\exp \left\{\int_{0}^{\infty}\left(\mathrm{e}^{-t}-\mathrm{e}^{-q t}\right) t^{-1} \mathbf{P}^{0}\left(X_{t}<0\right) d t\right\} \\
& =\exp \left\{\int_{0}^{\infty}\left(\mathrm{e}^{-t}-\mathrm{e}^{-q t}\right) t^{-1}\left(1-\mathbf{P}^{0}\left(X_{t} \geq 0\right)\right) d t\right\} \\
& =q / \Phi(q)
\end{aligned}
$$

where the last equality follows from the Frullani integral. As a consequence, (8.4) yields

$$
\int_{0}^{\infty} \mathrm{e}^{-q t} \mathbf{P}^{0}\left(X_{t} \geq 0\right) d t=\Phi^{\prime}(q) / \Phi(q)=\Phi^{\prime}(q) \widehat{\Phi}(q) / q .
$$

We know from Proposition 8.2 that $\Phi$ is regularly varying at $\infty$ with index $\alpha$, and also that $\widehat{\Phi}$ is regularly varying at $\infty$ with index $1-\alpha$. Because $\Phi$ and $\widehat{\Phi}$ are Laplace exponents of subordinators with zero drift, we obtain from the LévyKhintchine formula that

$$
\Phi^{\prime}(q)=\int_{(0, \infty)} \mathrm{e}^{-q x} x d(-\bar{\Pi}(x)) \quad, \quad \widehat{\Phi}(q) / q=\int_{0}^{\infty} \mathrm{e}^{-q x} \overline{\widehat{\Pi}}(x) d x,
$$


where $\bar{\Pi}$ (respectively, $\widehat{\widehat{\Pi}}$ ) is the tail of the Lévy measure of the ladder time process of $X$ (respectively, of $\widehat{X}$ ). We now get from (8.7)

$$
\mathbf{P}^{0}\left(X_{t} \geq 0\right)=\int_{(0, t)} \overline{\widehat{\Pi}}(t-s) s d(-\bar{\Pi}(s)) \quad \text { for a.e. } t>0 .
$$

By a change of variables, the right-hand side can be re-written as

$$
t \int_{(0,1)} \overline{\widehat{\Pi}}(t(1-u)) u d(-\bar{\Pi}(t u))=\int_{(0,1)} \frac{\overline{\widehat{\Pi}}(t(1-u))}{\widehat{\Phi}(1 / t)} u d\left(-\frac{\bar{\Pi}(t u)}{\Phi(1 / t)}\right) .
$$

Now, apply the second part of Proposition 1.5. For every fixed $\varepsilon \in(0,1)$, we have uniformly on $u \in[\varepsilon, 1-\varepsilon]$ as $t \rightarrow 0+$ :

$$
\frac{\bar{\Pi}(t u)}{\Phi(1 / t)} \rightarrow \frac{u^{-\alpha}}{\Gamma(1-\alpha)} \quad, \quad \frac{\overline{\widehat{\Pi}}(t(1-u))}{\widehat{\Phi}(1 / t)} \rightarrow \frac{(1-u)^{\alpha-1}}{\Gamma(\alpha)} .
$$

Recall $\mathbf{P}^{0}\left(X_{t} \geq 0\right)$ depends continuously on $t>0$. We deduce from (8.8) that

$$
\liminf _{t \rightarrow 0+} \mathbf{P}^{0}\left(X_{t} \geq 0\right) \geq \frac{\alpha}{\Gamma(\alpha) \Gamma(1-\alpha)} \int_{\varepsilon}^{1-\varepsilon}(1-u)^{\alpha-1} u^{-\alpha} d u,
$$

and as $\varepsilon$ can be picked arbitrarily small, $\lim \inf _{t \rightarrow 0+} \mathbf{P}^{0}\left(X_{t} \geq 0\right) \geq \alpha$. The same argument for the dual process gives $\liminf _{t \rightarrow 0+} \mathbf{P}^{0}\left(X_{t}<0\right) \geq 1-\alpha$, which establishes $(8.5)$.

If, as usual, we denote by $\sigma$ the inverse local time at 0 of the reflected process $S-X$, then it is easy to check from the stationarity and independence of the increments of $X$ that the time-changed process $H=X \circ \sigma=S \circ \sigma$ is again a subordinator. One calls $H$ the ladder height process; it has several interesting applications in fluctuation theory for Lévy processes. We refer to sections 4 and 5 of chapter VI in [11] for more on this topic.

\subsection{The spectrally negative case}

Throughout this section, we suppose that the real-valued Lévy process $X$ has no positive jumps, one sometimes says that $X$ is spectrally negative. The degenerate case when either $X$ is the negative of a subordinator or a deterministic drift has no interest and will be implicitly excluded in the sequel. We refer to Chapter VII in [11] for a detailed account of the theory of such processes.

The absence of positive jumps enables to use the same argument as that in section 1.1 to show that the first passage process of $X$

$$
\sigma_{t}=\inf \left\{s \geq 0: X_{s}>t\right\} \quad(t \geq 0)
$$

is a subordinator. The inverse of $\sigma$ coincides with the (continuous) supremum process $S$ of $X$, so $S$ serves as a local time on the set of times when $X$ reaches a new supremum, 
that when $S=X$. In other words, $S$ is proportional to the local time at 0 of the reflected process $S-X$.

As usual, we denote the Laplace exponent of $\sigma$ by $\Phi$. Note that if $T$ stands for an independent exponential time, say with parameter $q>0$, then

$$
\mathbf{P}^{0}\left(S_{T}>x\right)=\mathbf{P}^{0}\left(\sigma_{x}<T\right)=\mathbf{E}^{0}\left(\exp \left\{-q \sigma_{x}\right\}\right)=\mathrm{e}^{-x \Phi(q)}
$$

for every $x>0$, so that $S_{T}$ has an exponential distribution with parameter $\Phi(q)$. By taking $q$ sufficiently large, we see that for every fixed $t>0, S_{t}$ has a finite exponential moment of any order. As a consequence, though $X$ may take values of both signs, its exponential moments are finite. This enables us to study $X$ using the Laplace transform instead of the Fourier transform. More precisely, the characteristic exponent can be continued analytically on the lower half-plane $\{z \in \mathbb{C}: \Im(z)<0\}$. We then put $\psi(\lambda)=\Psi(-\mathrm{i} \lambda)$ for $\lambda>0$, so that

$$
\mathbf{E}^{0}\left(\exp \left\{\lambda X_{t}\right\}\right)=\exp \{t \psi(\lambda)\}, \quad \lambda \geq 0 .
$$

Invoking Hölder's inequality, we see that the mapping $\psi:[0, \infty) \rightarrow(-\infty, \infty)$ is strictly convex. On the other hand, we also deduce from the monotone convergence theorem that $\lim _{\lambda \rightarrow \infty} \psi(\lambda)=\infty$.

We are now able to specify the Laplace exponent $\Phi$.

Proposition 8.4 We have $\Phi \circ \psi(\lambda)=\lambda$ for every $\lambda \geq 0$ such that $\psi(\lambda)>0$.

Proof: It follows from the independence and stationarity of the increments that the process

$$
\exp \left\{\lambda X_{s}-\psi(\lambda) s\right\}, \quad s \geq 0
$$

is a nonnegative martingale. As $X$ cannot jump above the level $t$, we must have $X_{\sigma_{t}}=t$ on $\left\{\sigma_{t}<\infty\right\}$. On the other hand, the assumption that $\psi(\lambda)>0$ ensures that the martingale converges a.s. to 0 as $s \rightarrow \infty$ on the event $\left\{\sigma_{t}=\infty\right\}$. An application of the optional sampling theorem at the stopping time $\sigma_{t}$ yields

$$
\mathbf{E}^{0}\left(\exp \left\{\lambda t-\psi(\lambda) \sigma_{t}\right\}, \sigma_{t}<\infty\right)=1 .
$$

Recall the convention $\mathrm{e}^{-\infty}=0$; the preceding identity can be re-written as

$$
\exp \{-\lambda t\}=\mathbf{E}^{0}\left(\exp \left\{-\psi(\lambda) \sigma_{t}\right\}\right)=\exp \{-t \Phi(\psi(\lambda))\},
$$

which establishes our claim.

In comparison with (8.4), Proposition 8.4 provides an explicit expression for the Laplace exponent $\Phi$ directly in terms of our data (namely, $\psi$ ) which is much easier to deal with. For instance, it is immediately seen that $\Phi$ is regularly varying with index $\rho \in[0,1]$ if and only if $\psi$ is regularly varying with index $1 / \rho$ (which forces in fact $\rho$ to be greater than or equal to $1 / 2$ ). In the same vein, the lower and upper indices of $\Phi$ are given by

$$
\begin{aligned}
& \text { ind }(\Phi)=\sup \left\{\rho>0: \lim _{\lambda \rightarrow \infty} \psi(\lambda) \lambda^{-1 / \rho}=0\right\} \\
& \overline{\operatorname{ind}}(\Phi)=\inf \left\{\rho>0: \lim _{\lambda \rightarrow \infty} \psi(\lambda) \lambda^{-1 / \rho}=\infty\right\} .
\end{aligned}
$$


As another example of application, we derive the following extension of Khintchine's law of the iterated logarithm (see also [10] for further results in the same vein).

Corollary 8.5 There is a positive constant c such that

$$
\limsup _{t \rightarrow 0+} \frac{X_{t} \Phi\left(t^{-1} \log |\log t|\right)}{\log |\log t|}=c \quad \text { a.s. }
$$

Proof: Consider the functions

$$
f(t)=\frac{\Phi\left(t^{-1} \log \log \Phi\left(t^{-1}\right)\right)}{\log \log \Phi\left(t^{-1}\right)} \quad \text { and } \quad \tilde{f}(t)=\frac{\Phi\left(t^{-1} \log \log \Phi\left(t^{-1}\right)\right)}{\log \log \Phi\left(t^{-1} \log \log \Phi\left(t^{-1}\right)\right)} .
$$

The function $s \rightarrow s / \log \log s$ is monotone increasing on some neighbourhood of $\infty$ and the function $t \rightarrow \Phi\left(t^{-1} \log \log \Phi\left(t^{-1}\right)\right)$ decreases. We deduce that the compound function $\tilde{f}$ decreases on some neighbourhood of 0 . Moreover, it is easily seen that

$$
\log \log \Phi\left(t^{-1} \log \log \Phi\left(t^{-1}\right)\right) \sim \log \log \Phi\left(t^{-1}\right)
$$

(cf. the proof of Lemma 4.2), so that $f(t) \sim \tilde{f}(t)$ as $t \rightarrow 0+$.

Because the supremum process $S$ is proportional to the local time at 0 of $S-X$, we deduce from Theorem 4.1 that $\lim _{\sup _{t \rightarrow 0+}} S_{t} \tilde{f}(t)=c$ a.s. for some positive constant c. By an obvious argument of monotonicity, we may replace $S$ by $X$ in the preceding identity. So all that we need now is to check that

$$
\tilde{f}(t) \sim \frac{\Phi\left(t^{-1} \log |\log t|\right)}{\log |\log t|} \quad(t \rightarrow 0+) .
$$

On the one hand, it is easily seen from the Lévy-Khintchine formula for $\psi$ that $\lim \sup _{\lambda \rightarrow \infty} \lambda^{-2} \psi(\lambda)<\infty$, which in turn implies that $\liminf _{\lambda \rightarrow \infty} \lambda^{-1 / 2} \Phi(\lambda)>0$. On

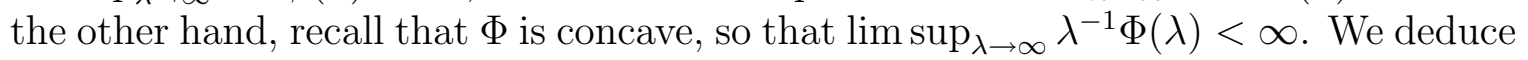
that

$$
\log \log \Phi\left(t^{-1}\right) \sim \log |\log t| \quad \text { as } t \rightarrow 0+,
$$

and then, since $\Phi$ is concave and increasing, that

$$
\Phi\left(t^{-1} \log \log \Phi\left(t^{-1}\right)\right) \sim \Phi\left(t^{-1} \log |\log t|\right) \quad \text { as } t \rightarrow 0+.
$$

Our claim follows.

We refer to Jaffard [86] and the references therein for further results on the regularity of the paths of Levy processes, in particular precise information on their multifractal structure.

\subsection{Bochner's subordination for Lévy processes}

Bochner [25] introduced the concept of subordination (after which subordinators were named) of Markov processes as follows. Let $M=\left(\Omega, \mathcal{M}, \mathcal{M}_{t}, M_{t}, \theta_{t}, \mathbf{P}^{x}\right)$ be some timehomogeneous Markov process and $\sigma=\left(\sigma_{t}: t \geq 0\right)$ a subordinator that is independent 
of $M$. The process $\tilde{M}=\left(\tilde{M}_{t}=M_{\sigma_{t}}: t \geq 0\right)$ obtained from $M$ by time-substitution based on $\sigma$ (with the convention that $M_{\infty}=\Upsilon$ where $\Upsilon$ is a cemetery point for $M$ ) is referred to as the subordinate process of $M$ with directing process $\sigma$. It is easily seen that the homogeneous Markov property is preserved by this time-substitution, in the sense that the process $\tilde{M}=\left(\Omega, \mathcal{M}, \tilde{\mathcal{M}}_{t}, \tilde{M}_{t}, \tilde{\theta}_{t}, \mathbf{P}^{x}\right)$ is again Markovian, where $\tilde{\mathcal{M}}_{t}=\mathcal{M}_{\sigma_{t}}$ and $\tilde{\theta}_{t}=\theta_{\sigma_{t}}$. More precisely, the semigroup $\left(\tilde{Q}_{t}: t \geq 0\right)$ of $\tilde{M}$ is given in terms of the semigroup $\left(Q_{t}: t \geq 0\right)$ of $M$ and the distribution of $\sigma$ by

$$
\tilde{Q}_{t}(x, d y)=\int_{[0, \infty)} Q_{s}(x, d y) \mathbb{P}\left(\sigma_{s} \in d t\right) .
$$

We refer to Feller [53], Bouleau [27] and Hirsch [72] for more on this topic. See also Bakry [2], Jacob and Schilling [84, 85], Meyer [119] and the references therein for applications in analysis (in particular to the Riesz transform and the Paley-Wiener theory); and Bouleau and Lépingle [28] for applications to simulation methods.

We now consider the special case when the Markov process is a Lévy process, i.e. $M=X$. In order to avoid problems related to killing, we will also suppose that $\sigma$ is a strict subordinator. From an analytic viewpoint, this means that the semigroup $\left(Q_{t}: t \geq 0\right)$ is a Markovian convolution semigroup, namely

$$
Q_{t} f(x)=\int_{\mathbb{R}} f(x+y) \mathbf{P}^{0}\left(X_{t} \in d y\right)
$$

for every Borel bounded function $f$. It follows from (8.9) that $\left(\tilde{Q}_{t}: t \geq 0\right)$ is also a Markovian convolution semigroup, i.e. the subordinate process $\tilde{X}$ is again a Lévy process.

Because the law of a Lévy process is specified by the characteristic exponent $\Psi$, it is natural to search for an expression of the characteristic exponent $\tilde{\Psi}$ of the subordinate Lévy process $\tilde{X}$. To this end, observe first that $\Psi$ maps $\mathbb{R}$ into $\mathbb{C}_{+}=\{z \in \mathbb{C}: \Re z \geq 0\}$, and second (from the Lévy-Khintchine formula) that the Laplace exponent $\Phi$ of a subordinator can be continued analytically on $\mathbb{C}_{+}$; we will still denote by $\Phi$ this extension. It should be clear that

$$
\mathbb{E}\left(\mathrm{e}^{-z \sigma_{t}}\right)=\mathrm{e}^{-t \Phi(z)} \quad \text { for any } z \in \mathbb{C}_{+} .
$$

As $X$ and $\sigma$ are independent, we then get

$$
\mathbf{E}^{0}\left(\exp \left\{\mathrm{i} \lambda X_{\sigma_{t}}\right\}\right)=\mathbb{E}\left(\exp \left\{-\Psi(\lambda) \sigma_{t}\right\}\right)=\exp \{-t \Phi(\Psi(\lambda))\},
$$

which proves the following statement:

Proposition 8.6 (Bochner [25]) Let $X$ be a Lévy process with characteristic exponent $\Psi$ and $\sigma$ an independent subordinator with Laplace exponent $\Phi$. Then the subordinate process $\tilde{X}=X \circ \sigma$ is a Lévy process with characteristic exponent

$$
\tilde{\Psi}=\Phi \circ \Psi .
$$

We refer to the second chapter of Chateau [34] for a study of the so-called subordination process, in which the subordinator $\sigma$ is viewed as a parameter. 
We now quote without proof a result of Huff [80], who has been able to make explicit the Lévy-Khintchine formula (8.1) for the subordinate process $\tilde{X}$. In the obvious notation, we have

$$
\begin{gathered}
\tilde{a}=\mathrm{d} a+\int_{(0, \infty)} \mathbf{E}^{0}\left(X_{t},\left|X_{t}\right|<1\right) \Pi(d t) \quad, \quad \tilde{b}=\mathrm{d} b, \\
\tilde{\Lambda}(d x)=\mathrm{d} \Lambda(d x)+\int_{(0, \infty)} \mathbf{P}^{0}\left(X_{t} \in d x\right) \Pi(d t) .
\end{gathered}
$$

Here is a classical example of Proposition 8.6 due to Spitzer [145]. Suppose that $(X, Y)$ is a planar Brownian motion and let $\sigma$ be the first-passage process of $Y$ (see Section 1.1). Thus, the characteristic exponent of $X$ is $\Psi(\lambda)=\frac{1}{2} \lambda^{2}$ for $\lambda \in \mathbb{R}$ and the Laplace exponent of $\sigma$ is $\Phi(q)=\sqrt{2 q}$ for $q \geq 0$. The characteristic exponent of the subordinate process $\tilde{X}=X \circ \sigma$ is thus $\tilde{\Psi}(\lambda)=|\lambda|$, i.e. $\tilde{X}$ is a standard symmetric Cauchy process. In the more general case when $\sigma$ is a stable subordinator of index $\alpha \in(0,1)$ independent of $X$, then $\tilde{X}$ is a symmetric stable process with index $2 \alpha$. See Molchanov and Ostrovski [121] and also Le Gall [107, 108] for connections with the so-called cone points of planar Brownian motion.

We now end this chapter with an application of the subordination technique to the so-called iterated Brownian motion. Consider $B^{+}=\left(B^{+}(t), t \geq 0\right), B^{-}=\left(B^{-}(t), t \geq\right.$ $0)$ and $B=\left(B_{t}, t \geq 0\right)$ three independent linear Brownian motions started from 0 . The process $Y=\left(Y_{t}, t \geq 0\right)$ given by

$$
Y_{t}=\left\{\begin{array}{cc}
B^{+}\left(B_{t}\right) & \text { if } B_{t} \geq 0 \\
B^{-}\left(-B_{t}\right) & \text { if } B_{t}<0
\end{array}\right.
$$

is called an iterated Brownian motion. Its study has been motivated by certain limit theorems and a connection with partial differential equations involving the square of the Laplacian, and has been undertaken by numerous authors (cf. Khoshnevisan and Lewis [99] for a list of references). Our purpose here is to investigate the supremum process of $Y$,

$$
\bar{Y}_{t}=\sup \left\{Y_{s}: 0 \leq s \leq t\right\} \quad(t \geq 0)
$$

via Bochner's subordination. To this end, we consider the supremum processes $S^{+}$, $S^{-}, S$ and $I$, of $B^{+}, B^{-}, B$ and $-B$, respectively. Observing that

$$
S^{+}\left(S_{t}\right)=\sup \left\{Y_{s}: 0 \leq s \leq t \text { and } B_{t} \geq 0\right\},
$$

and a similar relation for $S^{-}\left(I_{t}\right)$, we see that the study of $\bar{Y}$ reduces to that of the compound processes $S^{+} \circ S$ and $S^{-} \circ I$, via the identity

$$
\bar{Y}=\left(S^{+} \circ S\right) \vee\left(S^{-} \circ I\right)
$$

Next, we introduce the right-continuous inverse of $S, \sigma=\inf \left\{s: S_{s}>\cdot\right\}$, and recall that $\sigma$ is a stable subordinator with index $1 / 2$, more precisely with Laplace exponent $\Phi(\lambda)=\sqrt{2 \lambda}$. The inverse $\sigma^{+}$of $S^{+}$has the same law as $\sigma$ and is independent of $\sigma$. By an immediate variation of Proposition 8.6 (involving Laplace transform instead of Fourier transform), $\tilde{\sigma}=\sigma \circ \sigma^{+}$is a subordinator with Laplace exponent $\tilde{\Phi}(\lambda)=(8 \lambda)^{1 / 4}$. Plainly $\sigma \circ \sigma^{+}$is the right-continuous inverse of $S^{+} \circ S$ and we 
conclude that the right-continuous inverse of the supremum of an iterated Brownian motion can be expressed as

$$
\inf \left\{t: \bar{Y}_{t}>\cdot\right\}=\sigma^{(1)} \wedge \sigma^{(2)}
$$

where $\sigma^{(1)}$ and $\sigma^{(2)}$ are both subordinators with Laplace exponent $\tilde{\Phi}$.

An application of the law of the iterated logarithm for the inverse of a stable subordinator (see Theorem 4.1) now gives

$$
\limsup \frac{S^{+} \circ S_{t}}{t^{1 / 4}(\log |\log t|)^{3 / 4}}=2^{5 / 4} 3^{-3 / 4} \quad \text { a.s. }
$$

both as $t \rightarrow 0+$ and $t \rightarrow \infty$. Using (8.10), one can replace $S^{+} \circ S_{t}$ by $\bar{Y}_{t}$ (or even by $\left.Y_{t}\right)$ in (8.11), which establishes the law of the iterated logarithm for the iterated Brownian motion proven previously by Csáki et al. [38] and Deheuvels and Mason [41] for large times, and by Burdzy [31] for small times. We refer to [12] for further applications of this technique. 


\section{Chapter 9}

\section{Occupation times of a linear Brownian motion}

We consider the occupation time process $A .=\int_{0} f\left(B_{s}\right) d s$ where $B$ is a linear Brownian motion and $f \geq 0$ a locally integrable function. The time-substitution based on the inverse of the local time of $B$ at 0 turns $A$ into a subordinator. This enables us to derive several interesting properties for the occupation time process and for linear diffusions.

\subsection{Occupation times and subordinators}

Let $B=\left(B_{t}, t \geq 0\right)$ be a one-dimensional Brownian motion started from 0 . To agree with the usual normalization, we call the process

$$
\ell_{t}=\lim _{\varepsilon \rightarrow 0+} \frac{1}{2 \varepsilon} \int_{0}^{t} \mathbf{1}_{\left\{\left|B_{s}\right|<\varepsilon\right\}} d s, \quad t \geq 0
$$

Lévy's local time ${ }^{1}$ of $B$ at 0 . Consider a locally integrable function $f: \mathbb{R} \rightarrow[0, \infty)$ and the corresponding occupation time process of $B$

$$
A_{t}=\int_{0}^{t} f\left(B_{s}\right) d s, \quad t \geq 0 .
$$

(More generally, we might have considered the additive functional associated with some Radon measure $\mu$, see e.g. section X.2 in Revuz and Yor [132], but for the sake of simplicity, we will stick to the case when $\mu(d x)=f(x) d x$ is absolutely continuous.)

Let $\tau(t)=\inf \left\{s: \ell_{s}>t\right\}$ be the right-continuous inverse of $\ell$. A routine argument based on the additivity, the fact that $\ell$ only increases on the zero-set of $B$ and the strong Markov property, shows that the time-changed process

$$
\sigma_{t}=A_{\tau(t)}=\int_{0}^{\tau(t)} f\left(B_{s}\right) d s, \quad t \geq 0
$$

is a subordinator.

\footnotetext{
${ }^{1}$ This means that the local time at 0 in the sense of section 2.2 is $L_{t}=2^{-1 / 2} \ell_{t}$, in order to agree with (2.1).
} 
Results on subordinators can be very useful in investigating occupation times. To this end we need information on the Laplace exponent $\Phi$ and the Lévy measure $\Pi$ of $\sigma$; and this motivates the next section.

\subsection{Lévy measure and Laplace exponent}

\subsubsection{Lévy measure via excursion theory}

Our first purpose is to express the Lévy measure of $\sigma$ in terms of Itô's excursion measure. The obvious hint for this is that, since the occupation time $A$ is a continuous process, the jumps of the subordinator $\sigma=A \circ \tau$ correspond to the increments of $A$ on the intervals of times when $B$ has an excursion away from 0 .

Recall the setting of section 3.2 and specialize it to the Brownian case. Let $n$ be the measure of the excursions of $B$ away from 0 , that is the characteristic measure of the Poisson point process

$$
e_{t}(s)=\left\{\begin{array}{cc}
B_{\tau(t-)+s} & \text { if } 0 \leq s<\tau(t)-\tau(t-) \\
0 & \text { otherwise }
\end{array}\right.
$$

We denote the generic excursion by $\epsilon=(\epsilon(s): s \geq 0)$ and its first return time to 0 by $\rho(\epsilon)=\inf \{s>0: \epsilon(s)=0\}$.

Proposition 9.1 The drift coefficient and the killing rate of $\sigma$ are $\mathrm{d}=0$ and $\mathrm{k}=0$, respectively. The Lévy measure of $\sigma$ coincides with the distribution of $\int_{0}^{\rho(\epsilon)} f(\epsilon(s)) d s$ under $n$, i.e.

$$
\Pi(d x)=n\left(\int_{0}^{\rho(\epsilon)} f(\epsilon(s)) d s \in d x\right) .
$$

Proof: We split the time interval $[0, \tau(1)]$ into excursion intervals. Since Brownian motion spends zero time at 0 , we have

$$
\begin{aligned}
\int_{0}^{\tau(1)} f\left(B_{s}\right) d s=\sum_{0 \leq t \leq 1} \int_{\tau(t-)}^{\tau(t)} f\left(B_{s}\right) d s & =\sum_{0 \leq t \leq 1} \int_{0}^{\tau(t)-\tau(t-)} f\left(B_{\tau(t-)+s}\right) d s \\
& =\sum_{0 \leq t \leq 1} \int_{0}^{\rho\left(e_{t}\right)} f\left(e_{t}(s)\right) d s
\end{aligned}
$$

where $e=\left(e_{t}: t \geq 0\right)$ is the excursion process (see above). Applying the exponential formula for Poisson point processes (see e.g. Proposition 12 in section XII.1 in [132]), we get

$$
\begin{aligned}
& \mathbf{E}^{0}\left(\exp \left\{-\lambda \sum_{0 \leq t \leq 1} \int_{0}^{\rho\left(e_{t}\right)} f\left(e_{t}(s)\right) d s\right\}\right) \\
= & \exp \left\{-n\left(1-\exp \left\{-\lambda \int_{0}^{\rho(\epsilon)} f(\epsilon(s)) d s\right\}\right)\right\} \\
= & \exp \left\{-\int_{(0, \infty)}\left(1-\mathrm{e}^{-\lambda x}\right) n\left(\int_{0}^{\rho(\epsilon)} f(\epsilon(s)) d s \in d x\right)\right\} .
\end{aligned}
$$


Comparison with the Lévy-Khintchine formula establishes the claim.

Another useful observation which stems from excursion theory is the following independence property.

Corollary 9.2 Let $f_{+}, f_{-}: \mathbb{R} \rightarrow[0, \infty)$ be two locally integrable functions with $\operatorname{Supp}\left(f_{+}\right) \subseteq[0, \infty)$ and $\operatorname{Supp}\left(f_{-}\right) \subseteq(-\infty, 0]$, respectively. Then the subordinators

$$
\sigma_{t}^{+}=\int_{0}^{\tau(t)} f_{+}\left(B_{s}\right) d s \quad \text { and } \quad \sigma_{t}^{+}=\int_{0}^{\tau(t)} f_{-}\left(B_{s}\right) d s
$$

are independent. If moreover $f_{-}(x)=f_{+}(-x)$, then $\sigma^{+}$and $\sigma^{-}$have the same law.

Proof: We know from the foregoing that $\sigma^{+}$and $\sigma^{-}$are two subordinators in the same filtration, both with zero drift and zero killing rate. They are determined by their jump processes. Since jumps correspond to increments of the occupation times on an interval of excursion of $B$ away from $0, \sigma^{+}$jumps only when the excursion process $e$ takes values in the space of nonnegative paths, whereas $\sigma^{-}$jumps only when $e$ takes values in the space of non-positive paths. In particular, $\sigma^{+}$and $\sigma^{-}$never jump simultaneously. By a well-known property of Poisson point processes, their respective jump processes are independent. Because $\sigma^{+}$and $\sigma^{-}$are both characterized by their jumps, they are independent.

Finally, the excursion measure is symmetric, that is $n$ is invariant by the mapping $\epsilon \rightarrow-\epsilon$. It follows that $\sigma^{+}$and $\sigma^{-}$have the same Lévy measure, and hence the same law, whenever $f_{-}(x)=f_{+}(-x)$.

\subsubsection{Laplace exponent via the Sturm-Liouville equation}

The main result of this subsection characterizes the Laplace exponent $\Phi$ in terms of the solution of a Sturm-Liouville equation.

Proposition 9.3 For every $\lambda>0$, there exists a unique function $y_{\lambda}: \mathbb{R} \rightarrow[0,1]$ such that:

- $y_{\lambda}$ is a convex increasing function on $(-\infty, 0)$, and a convex decreasing function on $(0, \infty)$.

- $y_{\lambda}$ solves the Sturm-Liouville equation $y^{\prime \prime}=2 \lambda y f$ on both $(-\infty, 0)$ and $(0, \infty)$, and $y_{\lambda}(0)=1$.

The Laplace exponent of $\sigma$ is then given by

$$
\Phi(\lambda)=\frac{1}{2}\left(y_{\lambda}^{\prime}(0-)-y_{\lambda}^{\prime}(0+)\right) .
$$

Proof: We present a proof due to Jeulin and Yor [89], which is based on stochastic calculus. One can also establish the result by analytic arguments that rely on the Feynman-Kac formula and Proposition 2.2; see e.g. Itô and McKean [83], Jeanblanc et al. [88] and Pitman and Yor [128]. 
The existence and uniqueness of $y_{\lambda}$ is a well-known result on the Sturm-Liouville equation; see for instance Dym and McKean [49]. By stochastic calculus (more precisely, by an application of the Itô-Tanaka formula), the process

$$
M_{t}=y_{\lambda}\left(B_{t}\right) \exp \left\{\frac{1}{2}\left(y_{\lambda}^{\prime}(0-)-y_{\lambda}^{\prime}(0+)\right) \ell_{t}-\lambda \int_{0}^{t} f\left(B_{s}\right) d s\right\}, \quad t \geq 0,
$$

is a local martingale. Because $M_{s} \leq \exp \left\{\frac{1}{2}\left(y_{\lambda}^{\prime}(0-)-y_{\lambda}^{\prime}(0+)\right) t\right\}$ for every $s \leq \tau(t)$, we can apply Doob's optional sampling theorem for $M$ at time $\tau(t)$. Since $\ell_{\tau(t)}=t$ and $B_{\tau(t)}=0$, we get

$$
\mathbf{E}^{0}\left(\exp \left\{\frac{1}{2}\left(y_{\lambda}^{\prime}(0-)-y_{\lambda}^{\prime}(0+)\right) t-\lambda \int_{0}^{\tau(t)} f\left(B_{s}\right) d s\right\}\right)=1,
$$

that is

$$
\exp \{-t \Phi(\lambda)\}=\mathbf{E}^{0}\left(\exp \left\{-\lambda \int_{0}^{\tau(t)} f\left(B_{s}\right) d s\right\}\right)=\exp \left\{-\frac{1}{2}\left(y_{\lambda}^{\prime}(0-)-y_{\lambda}^{\prime}(0+)\right) t\right\} .
$$

This completes the proof.

The solutions of Sturm-Liouville equations are not explicitly known in general (see however the hand-book by Borodin and Salminen [26] for a number of explicit formulas in some important special cases). Nonetheless one can deduce handy bounds for the Laplace exponent $\Phi$ in terms of the function $f$ that will be quite useful in the sequel.

Corollary 9.4 Put $F(x)=\int_{0}^{x} f(t) d t(x \in \mathbb{R})$ and

$$
G(t)=2 \int_{0}^{t}(F(x)-F(-x)) d x, \quad t \geq 0,
$$

so $G$ in a convex increasing function on $[0, \infty)$. We write $H(s)=\inf \{t \geq 0: G(t)>$ $s\}, s \geq 0$, for its inverse. Then we have

$$
\Phi(\lambda) \asymp \frac{1}{H(1 / \lambda)} .
$$

As a consequence, if $U$ stands for the renewal measure of $\sigma$ and $I$ for the integrated tail of its Lévy measure (c.f. Lemma 1.4), then

$$
U(x) \asymp H(x) \quad \text { and } \quad I(x) \asymp \frac{x}{H(x)}
$$

Proof: (i) We first suppose that $f$ vanishes on $(-\infty, 0)$ and start with the integral Sturm-Liouville equation:

$$
y_{\lambda}(x)=1+x y_{\lambda}^{\prime}(0+)+2 \lambda \int_{0}^{x}\left(\int_{0}^{t} y_{\lambda}(s) f(s) d s\right) d t, \quad x \geq 0, \lambda>0 .
$$

Using the fact that $0 \leq y_{\lambda} \leq 1$, we deduce the inequality

$$
-x y_{\lambda}^{\prime}(0+) \leq 1+2 \lambda \int_{0}^{x}\left(\int_{0}^{t} f(s) d s\right) d t=1+\lambda G(x) .
$$


Using this with $x=H(1 / \lambda)$ gives $-y_{\lambda}^{\prime}(0+) H(1 / \lambda) \leq 2$.

To establish an lowerbound, we use the fact that $y_{\lambda}$ decreases on $[0, \infty)$ in $(9.1)$ to get

$$
y_{\lambda}(x)-x y_{\lambda}^{\prime}(0+) \geq 1+2 \lambda \int_{0}^{x}\left(\int_{0}^{t} y_{\lambda}(x) f(s) d s\right) d t=1+\lambda y_{\lambda}(x) G(x) .
$$

Specifying this for $x=H(1 / \lambda)$ gives $-y_{\lambda}^{\prime}(0+) H(1 / \lambda) \geq 1$.

We have thus established that

$$
1 \leq-y_{\lambda}^{\prime}(0+) H(1 / \lambda) \leq 2, \quad \lambda>0
$$

in the special case when $f$ vanishes on $(-\infty, 0)$. By a symmetry argument, the bounds

$$
1 \leq y_{\lambda}^{\prime}(0-) H(1 / \lambda) \leq 2, \quad \lambda>0
$$

hold when $f$ vanishes on $(0, \infty)$. It is immediate to deduce that

$$
\frac{1}{H(1 / \lambda)} \asymp y_{\lambda}^{\prime}(0-)-y_{\lambda}^{\prime}(0+)
$$

in the general case; and our statement then derives from Proposition 9.3.

(ii) The estimate for the renewal measure now follows from Lemma 1.4. Since we know that the drift of $\sigma$ is zero, the second estimate also follows from Lemma 1.4.

A sharper estimate for $\Phi$ has been obtained in the form of a Tauberian type theorem by Kasahara ${ }^{2}$, under the condition that the indefinite integral $F$ of $f$ is regularly varying. We quote the result for completeness and refer to Kotani and Watanabe [102] on page 240 for details of the proof. Thanks to Corollary 9.2, we may restrict our attention to the case when $f$ vanishes on $(-\infty, 0)$.

Proposition 9.5 Suppose that $f \equiv 0$ on $(-\infty, 0)$. Then $\Phi$ is regularly varying at $0+$ (respectively, at $\infty$ ) with index $\alpha \in(0,1)$ if and only if $F$ is regularly varying at $\infty$ (respectively, at $0+$ ) with index $(1 / \alpha)-1$. In that case,

$$
\Phi(\lambda) \sim(\alpha(1-\alpha))^{\alpha} \frac{\Gamma(1-\alpha)}{\Gamma(1+\alpha)} \lambda^{\alpha} l(1 / \lambda) \quad(\lambda \rightarrow 0+),
$$

where $l$ is a slowly varying function at $\infty$ (respectively, at $0+$ ) such that an asymptotic inverse of $x \rightarrow x F(x)$ is $x \rightarrow x^{\alpha} l(x)$.

\subsubsection{Spectral representation of the Laplace exponent}

The so-called spectral theory of vibrating strings, which has been chiefly developed by M. G. Krein and his followers, is a most powerful tool for investigating the SturmLiouville boundary value problem that appears in Proposition 9.3. In this subsection, we will merely state the -tiny- portion of the theory that will be useful for the applications we have in mind; and refer to Dym and McKean [49] for a complete exposition.

\footnotetext{
${ }^{2}$ There is a typographical error in the definition of the constant $D_{\alpha}$ on p. 70 of [92]; see KotaniWatanabe [102].
} 
Proposition 9.6 (Krein) Let $y_{\lambda}$ be the function which appears in Proposition 9.3.

(i) There exists a unique measure $\nu$ on $[0, \infty)$ with $\int_{[0, \infty)}(1+\xi)^{-1} \nu(d \xi)<\infty$, such that for every $\lambda>0$ :

$$
\frac{2}{y_{\lambda}^{\prime}(0-)-y_{\lambda}^{\prime}(0+)}=\int_{[0, \infty)} \frac{\nu(d \xi)}{\lambda+\xi}
$$

(ii) There exists a unique measure $\widehat{\nu}$ on $[0, \infty)$ with $\int_{[0, \infty)}(1+\xi)^{-1} \widehat{\nu}(d \xi)<\infty$, such that for every $\lambda>0$ :

$$
\frac{y_{\lambda}^{\prime}(0-)-y_{\lambda}^{\prime}(0+)}{2 \lambda}=\int_{[0, \infty)} \frac{\widehat{\nu}(d \xi)}{\lambda+\xi} .
$$

When $f$ vanishes on $(-\infty, 0)$, the measure $f(x) d x$ is sometimes called a string (in fact Krein's theory deals with a completely general family of measures). The measure $\frac{1}{2} \nu$ in Proposition 9.6(i) is then known as the spectral measure of the string, and the measure $2 \widehat{\nu}$ in (ii) coincides with the spectral measure of the so-called dual string $d \widehat{F}(x)$, where $\widehat{F}$ is the right continuous inverse of the distribution function $F(x)=\int_{0}^{x} f(x) d x$.

Krein's theory yields the following remarkable formulas for the Laplace exponent $\Phi$ of $\sigma$ and the tail of its Lévy measure $\bar{\Pi}$, which seem to have been first observed by Knight [101] (see also Kotani and Watanabe [102] and Küchler [103]).

Corollary 9.7 Suppose that $f \equiv 0$ on $(-\infty, 0)$. We have

(i) There exists a unique measure $\nu$ on $[0, \infty)$ with $\int_{[0, \infty)}(1+\xi)^{-1} \nu(d \xi)<\infty$ such that

$$
\frac{1}{\Phi(\lambda)}=\int_{[0, \infty)} \frac{\nu(d \xi)}{\lambda+\xi}, \quad \lambda>0
$$

As a consequence, the renewal measure $U(d x)$ of $\sigma$ is absolutely continuous with density u given by

$$
u(x)=\int_{[0, \infty)} \mathrm{e}^{-x \xi} \nu(d \xi), \quad x>0 .
$$

(ii) There exists a unique measure $\widehat{\nu}$ on $[0, \infty)$ with $\int_{[0, \infty)}(1+\xi)^{-1} \widehat{\nu}(d \xi)<\infty$ such that

$$
\bar{\Pi}(x)=\int_{[0, \infty)} \mathrm{e}^{-x \xi} \widehat{\nu}(d \xi), \quad x>0
$$

Proof: (i) The first assertion follows immediately from Propositions 9.3 and 9.6. To get the second, just recall that the Laplace transform of the renewal measure is $1 / \Phi$, so that by Fubini's theorem

$$
\int_{[0, \infty)} \mathrm{e}^{-\lambda x} U(d x)=\int_{[0, \infty)} \frac{\nu(d \xi)}{\lambda+\xi}=\int_{0}^{\infty} \mathrm{e}^{-\lambda x}\left(\int_{[0, \infty)} \mathrm{e}^{-x \xi} \nu(d \xi)\right) d x
$$

(ii) Recall that $\sigma$ has zero drift. By an integration by parts in the Lévy-Khintchine formula, we get

$$
\begin{aligned}
\int_{0}^{\infty} \mathrm{e}^{-\lambda x} \bar{\Pi}(x) d x=\frac{\Phi(\lambda)}{\lambda} & =\frac{y_{\lambda}^{\prime}(0-)-y_{\lambda}^{\prime}(0+)}{2 \lambda} \quad \text { (by Proposition 9.3) } \\
& =\int_{[0, \infty)} \frac{\widehat{\nu}(d \xi)}{\lambda+\xi} \quad \text { (by Proposition } 9.6 \text { (ii)) } \\
& =\int_{0}^{\infty} \mathrm{e}^{-\lambda x}\left(\int_{[0, \infty)} \mathrm{e}^{-\xi x} \widehat{\nu}(d \xi)\right) d x \quad \text { (by Fubini) } .
\end{aligned}
$$


As the tail of the Lévy measure is decreasing and the Laplace transform of the spectral measure continuous, this establishes our claim.

In particular, the renewal measure and the Lévy measure both have completely monotone densities (Hawkes [71] observed that these two properties are equivalent for any subordinator). It seems there is no purely probabilistic proof for this remarkable feature.

It is immediately checked that $x \rightarrow \log u(x)$ is a decreasing convex function on $(0, \infty)$. In particular, the renewal density can also be expressed in the form

$$
u(x)=c \exp \left\{\int_{x}^{1} \bar{\mu}(t) d t\right\}
$$

for some decreasing locally integrable function $\bar{\mu}:(0, \infty) \rightarrow \mathbb{R}$. In other words, $\bar{\mu}$ is the tail of some measure on $(0, \infty)$, and the comparison with Theorem 7.2 shows that the range of $\sigma$ can be thought of as the set left uncovered by certain random intervals issued from a Poisson point process with characteristic measure $\mu$. It would be quite interesting to have probabilistic evidence of this fact.

\subsection{The zero set of a one-dimensional diffusion}

The material developed in the preceding section can be applied to the study of the zero set of a regular linear diffusion in natural scale ${ }^{3}$, using Feller's construction that we now recall.

For the sake of simplicity, we focus on the case when the speed measure is absolutely continuous, though this restriction is in fact superfluous. So let $f \geq 0$ be a locally integrable function such that the support of $f$ is an interval which contains the origin. The occupation time process $A_{t}=\int_{0}^{t} f\left(B_{s}\right) d s$ increases exactly when the Brownian motion $B$ visits $\operatorname{Supp}(f)$ and the time-changed process

$$
X_{t}=B_{\alpha(t)}, \quad t \geq 0, \quad \text { where } \alpha(t)=\inf \left\{s: A_{s}>t\right\},
$$

is a continuous Markov process. One calls $X=\left(X_{t}, t \geq 0\right)$ the $\operatorname{diffusion}$ in $\operatorname{Supp}(f)$ with natural scale and speed measure $f(x) d x$. Its infinitesimal generator is $\mathcal{G} g=$ $\frac{1}{2} g^{\prime \prime} / f$ with the Neumann reflecting condition at the boundary.

When one time-changes Lévy's local time $\ell$ of the Brownian motion by $\alpha$, one obtains a continuous increasing process which increases exactly on the zero set of $X$. Using the approximation

$$
\ell_{\alpha(t)}=\lim _{\varepsilon \rightarrow 0+} \frac{1}{2 \varepsilon} \int_{0}^{\alpha(t)} \mathbf{1}_{\left\{\left|B_{s}\right|<\varepsilon\right\}} d s=\lim _{\varepsilon \rightarrow 0+} \frac{1}{2 \varepsilon} \int_{0}^{t} \mathbf{1}_{\left\{\left|X_{s}\right|<\varepsilon\right\}} \frac{1}{f\left(X_{s}\right)} d s,
$$

we see that $\ell_{\alpha(\cdot)}$ is an additive functional of the diffusion. Hence, the local time $L$ of $X$ at 0 must be $L .=c \ell_{\alpha(\cdot)}$ for some normalizing constant $c>0$. We thus have

$$
L^{-1}(t)=\inf \left\{s \geq 0: L_{s}>t\right\}=\inf \left\{s \geq 0: \ell_{\alpha(s)}>t / c\right\}=A_{\tau(t / c)} .
$$

\footnotetext{
${ }^{3}$ Since we are only concerned with the zero set of the diffusion, this induces no loss of generality.
} 
In other words, the inverse local time of the diffusion coincides with the subordinator $\sigma$ up to a linear time-substitution.

As a first example of application, we present an explicit formula for the fractal dimensions of the zero set of the diffusion $X$ in terms of its speed measure. Recall from Theorem 5.1 that the fractal dimensions are given by the lower and upper indices of the Laplace exponent.

Corollary 9.8 The Hausdorff and packing dimensions of $\mathcal{R}=\left\{t \geq 0: X_{t}=0\right\}$ are given by

$$
\begin{aligned}
& \operatorname{dim}_{\mathrm{H}}(\mathcal{R})=\sup \left\{\rho \leq 1: \lim _{x \rightarrow 0+} x^{1-1 / \rho}(F(x)-F(-x))=\infty\right\} \\
& \operatorname{dim}_{\mathrm{P}}(\mathcal{R})=\inf \left\{\rho \leq 1: \lim _{x \rightarrow 0+} x^{1-1 / \rho}(F(x)-F(-x))=0\right\}
\end{aligned}
$$

where $F(x)=\int_{0}^{x} f(t) d t$.

Proof: For the sake of conciseness, we shall only consider the Hausdorff dimension which coincides with the lower index

$$
\underline{\text { ind }}(\Phi)=\sup \left\{\rho \leq 1: \lim _{\lambda \rightarrow \infty} \Phi(\lambda) \lambda^{-\rho}=\infty\right\}
$$

(cf. chapter 3). We know from Corollary 9.4 that $\Phi(\lambda) \asymp 1 / H(1 / \lambda)$, where $H$ is the inverse function of the indefinite integral $G(x)=2 \int_{0}^{x}(F(t)-F(-t)) d t$. It follows immediately that

$$
\underline{\text { ind }}(\Phi)=\sup \left\{\rho \leq 1: \lim _{x \rightarrow 0+} G(x) x^{1 / \rho}=\infty\right\} .
$$

Finally, the obvious bound

$$
x(F(x / 2)-F(-x / 2)) \leq G(x) \leq 2 x(F(x)-F(-x))
$$

completes the proof.

As a second illustration, we will use features on random covering to derive a result originally due to Tomisaki [149], which provides an explicit test to decide whether two independent diffusion processes ever visit a given point simultaneously. We first introduce some notation.

Let $X=\left(X_{t}: t \geq 0\right)$ and $Y=\left(Y_{t}: t \geq 0\right)$ be two independent regular diffusions in natural scale; for the sake of simplicity, we shall assume that both $X$ and $Y$ start from 0 . Their speed measures are denoted by $d F_{X}$ and $d F_{Y}$, respectively; we also write for $t \geq 0$

$$
G_{X}(t)=2 \int_{0}^{t}\left(F_{X}(x)-F_{X}(-x)\right) d x \quad, \quad G_{Y}(t)=2 \int_{0}^{t}\left(F_{Y}(x)-F_{Y}(-x)\right) d x
$$

and $H_{X}$ and $H_{Y}$ for the inverse functions of $G_{X}$ and $G_{Y}$. Recall that $H_{X}$ and $H_{Y}$ are concave and increasing. 
Corollary 9.9 (Tomisaki [149]) (i) The probability of that $X_{t}=Y_{t}=$ for some $t>0$ equals one if

$$
\int_{0}^{1} H_{X}^{\prime}(t) H_{Y}^{\prime}(t) d t<\infty
$$

and 0 otherwise.

(ii) The probability of the event $\left\{X_{t}=Y_{t}=0\right.$ infinitely often as $\left.t \rightarrow \infty\right\}$ equals one if

$$
\int_{0}^{1} H_{X}^{\prime}(t) H_{Y}^{\prime}(t) d t<\infty \quad \text { and } \quad \int_{1}^{\infty} H_{X}^{\prime}(t) H_{Y}^{\prime}(t) d t=\infty
$$

and 0 otherwise.

Proof: Let $\mathcal{R}_{X}$ and $\mathcal{R}_{Y}$ be the zero sets of $X$ and $Y$, respectively. Denote by $\sigma_{X}$ the inverse local times of $X$ at 0 . According to the observation made at the end of subsection 8.2 .3 , the range $\mathcal{R}_{X}$ of $\sigma_{X}$ can be viewed as the set left uncovered by random intervals issued from a Poisson point process with characteristic measure $\mu_{X}$. Idem for $\mathcal{R}_{Y}$ with a characteristic measure $\mu_{Y}$. Because $X$ and $Y$ are independent, the intersection of their zero sets can thus be thought of as the closed subset of $[0, \infty)$ left uncovered by random intervals issued from a Poisson point process with characteristic measure $\mu_{X}+\mu_{Y}$.

(i) We apply Theorem 7.2. The probability that $\mathcal{R}_{X} \cap \mathcal{R}_{Y}$ reduces to $\{0\}$ is one if

$$
\int_{0}^{1} \exp \left\{\int_{t}^{1}\left(\bar{\mu}_{X}(s)+\bar{\mu}_{Y}(s)\right) d s\right\} d t=\infty
$$

and zero otherwise. Writing $u_{X}$ and $u_{Y}$ for the renewal density of $\mathcal{R}_{X}$ and $\mathcal{R}_{Y}$ and applying (7.1), we see that (9.2) is equivalent to

$$
\int_{0}^{1} u_{X}(t) u_{Y}(t) d t=\infty
$$

Recall from Corollary 9.7 that $u_{X}$ decreases, so the latter is also equivalent (in the obvious notation) to

$$
\int_{0}^{1} U_{X}(t) d\left(-u_{Y}(t)\right)=\infty
$$

Using then the estimate of Corollary 9.4(ii), we deduce that

$$
(9.2) \Longleftrightarrow \int_{0}^{1} H_{X}(t) d\left(-u_{Y}(t)\right)=\infty .
$$

Finally, integrate by parts and apply again Corollary 9.4(ii) to derive

$$
(9.2) \Longleftrightarrow \int_{0}^{1} H_{X}^{\prime}(t) H_{Y}^{\prime}(t) d t=\infty
$$

(ii) The proof rests upon similar arguments and Corollary 7.4.

In the literature, there exist many other examples of applications of the spectral representation of the Laplace exponent $\Phi$. See in particular Bertoin [7], Kasahara [92], Kent [94, 95], Knight [101], Kotani and Watanabe [102], Küchler [103], Küchler and Salminen [104], Tomisaki [149], Watanabe [150, 151] and references therein. 


\section{References}

[1] S. Aspandiiarov and J. F. Le Gall (1995). Some new classes of exceptional times of linear Brownian motion. Ann. Probab. 23, 1605-1626.

[2] D. Bakry (1984). Etude probabiliste des transformées de Riesz et de l'espace $H^{1}$ sur les sphères. In: Séminaire de Probabilités XVIII, Lecture Notes in Maths. 1059 pp. 197-218. Springer, Berlin.

[3] M. T. Barlow (1985). Continuity of local times for Lévy processes. $Z$. Wahrscheinlichkeitstheorie verw. Gebiete 69, 23-35.

[4] M. T. Barlow (1988). Necessary and sufficient conditions for the continuity of local time of Lévy processes. Ann. Probab. 16, 1389-1427.

[5] M. T. Barlow, E. A. Perkins and S. J. Taylor (1986). Two uniform intrinsic constructions for the local time of a class of Lévy processes. Illinois J. Math. 30, 19-65.

[6] C. Berg and G. Forst (1975). Potential theory on locally compact Abelian groups. Springer, Berlin.

[7] J. Bertoin (1989). Applications de la théorie spectrale des cordes vibrantes aux fonctionnelles additives principales d'un mouvement brownien réfléchi. Ann. Inst. Henri Poincaré 25, 307-323.

[8] J. Bertoin (1995). Some applications of subordinators to local times of Markov processes. Forum Math. 7, 629-644.

[9] J. Bertoin (1995). Sample path behaviour in connection with generalized arcsine laws. Probab. Theory Relat. Fields 103, 317-327.

[10] J. Bertoin (1995). On the local rate of growth of Lévy processes with no positive jumps. Stochastic Process. Appl. 55, 91-100.

[11] J. Bertoin (1996). Lévy processes. Cambridge University Press, Cambridge.

[12] J. Bertoin (1996). Iterated Brownian motion and stable $\left(\frac{1}{4}\right)$ subordinator. Stat. Prob. Letters 27, 111-114.

[13] J. Bertoin (1997). Regularity of the half-line for Lévy processes. Bull. Sci. Math. 121, 345-354. 
[14] J. Bertoin (1997). Regenerative embedding of Markov sets. Probab. Theory Relat. Fields 108, 559-571.

[15] J. Bertoin (1998). The inviscid Burgers equation with Brownian initial velocity. Comm. Math. Phys. 193, 397-406.

[16] J. Bertoin (1999). Intersection of independent regenerative sets. Probab. Theory Relat. Fields 114, 97-121.

[17] J. Bertoin and R. A. Doney (1997). Spitzer's condition for random walks and Lévy processes. Ann. Inst. Henri Poincaré 33, 167-178.

[18] J. Bertoin and S. Jaffard (1997). Solutions multifractales de l'équation de Burgers. Matapli 52, 19-28.

[19] N. H. Bingham (1975). Fluctuation theory in continuous time. Adv. Appl. Prob. 7, 705-766.

[20] N. H. Bingham, C. M. Goldie and J. L. Teugels (1987). Regular variation. Cambridge University Press, Cambridge.

[21] R. M. Blumenthal (1992). Excursions of Markov processes. Birkhäuser, Boston.

[22] R. M. Blumenthal and R. K. Getoor (1961). Sample functions of stochastic processes with independent increments. J. Math. Mech. 10, 493-516.

[23] R. M. Blumenthal and R. K. Getoor (1968). Markov processes and potential theory. Academic Press, New-York.

[24] R. M. Blumenthal and R. K. Getoor (1970). Dual processes and potential theory. Proc. 12th Biennal Seminar, Canad. Math. Congress, 137-156.

[25] S. Bochner (1955). Harmonic analysis and the theory of probability. University of California Press, Berkeley.

[26] A. N. Borodin and P. Salminen (1996). Handbook of Brownian motion - Facts and formulae. Birkhäuser, Basel.

[27] N. Bouleau (1984). Quelques résultats sur la subordination au sens de Bochner. In: Séminaire de Théorie du Potentiel 7, Lecture Notes in Maths. 1061 pp. 54-81. Springer, Berlin.

[28] N. Bouleau and D. Lépingle (1994). Numerical methods for stochastic processes. Wiley, New York.

[29] L. Breiman (1968). A delicate law of the iterated logarithm for non-decreasing stable processes. Ann. Math. Stat. 39, 1818-1824. [Correction id (1970). 41, 1126.]

[30] J. Bretagnolle (1971). Résultats de Kesten sur les processus à accroissements indépendants. In: Séminaire de Probabilités V, Lecture Notes in Maths. 191 pp. 21-36. Springer, Berlin. 
[31] K. Burdzy (1993). Some path properties of iterated Brownian motion. In: Seminar on stochastic processes 1992, pp. 67-87. Birkhäuser, Boston.

[32] J. M. Burgers (1974). The nonlinear diffusion equation. Dordrecht, Reidel.

[33] L. Carraro and J. Duchon (1998). Equation de Burgers avec conditions initiales à accroissements indépendants et homogènes. Ann. Inst. Henri Poincaré: analyse non-linéaire 15, 431-458.

[34] O. Chateau (1990). Quelques remarques sur les processus à accroissements indépendants et stationnaires, et la subordination au sens de Bochner. Thèse d'Université. Laboratoire de Probabilités de l'Université Pierre et Marie Curie.

[35] K. L. Chung (1982). Lectures from Markov processes to Brownian motion. Springer, Berlin.

[36] K. L. Chung and P. Erdős (1952). On the application of the Borel-Cantelli lemma. Trans. Amer. Math. Soc. 72, 179-186.

[37] J. D. Cole (1951). On a quasi-linear parabolic equation occuring in aerodynamics. Quart. Appl. Math. 9, 225-236.

[38] E. Csáki, M. Csörgő, A. Földes and P. Révész (1989). Brownian local time approximated by a Wiener sheet. Ann. Probab. 17, 516-537.

[39] E. Csáki, M. Csörgö, A. Földes and P. Révész (1992). Strong approximation of additive functionals. J. Theoretic. Prob. 5, 679-706.

[40] E. Csáki, P. Révész and J. Rosen (1998). Functional laws of the iterated logarithm for local times of recurrent random walks on $\mathbb{Z}^{2}$. Ann. Inst. Henri Poincaré 34, 545-563.

[41] P. Deheuvels and D. M. Mason (1992) A functional LIL approach to pointwise Bahadur-Kiefer theorems. In: Probability in Banach spaces 8 (eds R.M. Dudley, M.G. Hahn and J. Kuelbs) pp. 255-266. Birkhäuser, Boston.

[42] C. Dellacherie and P. A. Meyer (1975). Probabilités et potentiel, vol. I. Hermann, Paris.

[43] C. Dellacherie and P. A. Meyer (1980). Probabilités et potentiel, vol. II. Théorie des martingales. Hermann, Paris.

[44] C. Dellacherie and P. A. Meyer (1987). Probabilités et potentiel, vol. IV. Théorie du potentiel, processus de Markov. Hermann, Paris.

[45] C. Dellacherie, B. Maisonneuve and P. A. Meyer (1992). Probabilités et potentiel, vol. V. Processus de Markov, compléments de calcul stochastique. Hermann, Paris.

[46] C. Donati-Martin (1991). Transformation de Fourier et temps d'occupation browniens. Probab. Theory Relat. Fields 88, 137-166. 
[47] R. A. Doney (1995). Spitzer's condition and ladder variables in random walk. Probab. Theory Relat. Fields 101, 577-580.

[48] R. A. Doney (1997). One-sided local large deviation and renewal theorems in the case of infinite mean.Probab. Theory Relat. Fields 107, 451-465.

[49] H. Dym and H. P. McKean (1976). Gaussian processes, function theory and the inverse spectral problem. Academic Press.

[50] E. B. Dynkin (1961). Some limit theorems for sums of independent random variables with infinite mathematical expectation. In: Selected Translations Math. Stat. Prob. vol. 1, pp. 171-189. Inst. Math. Statistics Amer. Math. Soc.

[51] S. N. Evans (1987). Multiple points in the sample path of a Lévy process. Probab. Theory Relat. Fields 76, 359-367.

[52] K. Falconer (1990). Fractal Geometry. Mathematical foundations and applications. Wiley, Chichester.

[53] W. E. Feller (1971). An introduction to probability theory and its applications, 2nd edn, vol. 2. Wiley, New-York.

[54] P. J. Fitzsimmons, B. E. Fristedt and B. Maisonneuve (1985). Intersections and limits of regenerative sets. Z. Wahrscheinlichkeitstheorie verw. Gebiete $\mathbf{7 0}$, $157-173$.

[55] P. J. Fitzsimmons, B. E. Fristedt and L. A. Shepp (1985). The set of real numbers left uncovered by random covering intervals. Z. Wahrscheinlichkeitstheorie verw. Gebiete 70, 175-189.

[56] P. J. Fitzsimmons and T.S. Salisbury (1989). Capacity and energy for multiparameter Markov processes. Ann. Inst. Henri Poincaré 25, 325-350.

[57] B. E. Fristedt (1967). Sample function behaviour of increasing processes with stationary independent increments. Pac. J. Math. 21, 21-33.

[58] B. E. Fristedt (1974). Sample functions of stochastic processes with stationary, independent increments. In: Advances in Probability 3, pp. 241-396. Dekker, New-York.

[59] B. E. Fristedt (1979). Uniform local behavior of stable subordinators. Ann. Probab. 7, 1003-1013.

[60] B. E. Fristedt (1996). Intersections and limits of regenerative sets. In: Random Discrete Structures (eds. D. Aldous and R. Pemantle) pp. 121-151. Springer, Berlin.

[61] B. E. Fristedt and W. E. Pruitt (1971). Lower functions for increasing random walks and subordinators. Z. Wahrscheinlichkeitstheorie verw. Gebiete 18, 167182 .

[62] B. E. Fristedt and W. E. Pruitt (1972). Uniform lower functions for subordinators. Z. Wahrscheinlichkeitstheorie verw. Gebiete 24, 63-70. 
[63] B. E. Fristedt and S. J. Taylor (1983). Construction of local time for a Markov process. Z. Wahrscheinlichkeitstheorie verw. Gebiete 62, 73-112.

[64] B. E. Fristedt and S. J. Taylor (1992). The packing measure of a general subordinator. Probab. Theory Relat. Fields 92, 493-510.

[65] N. Gantert and O. Zeitouni (1998). Large and moderate deviations for the local time of a recurrent random walk on $\mathbb{Z}^{2}$. Ann. Inst. Henri Poincaré 34, 687-704

[66] R. K. Getoor (1979). Excursions of a Markov process. Ann. Probab. 7, 244-266.

[67] I. I. Gihman and A. V. Skorohod (1975). The theory of stochastic processes II. Springer, Berlin.

[68] P. E. Greenwood and J. W. Pitman (1980). Construction of local time and Poisson point processes from nested arrays. J. London Math. Soc. 22, 182-192.

[69] J. Hawkes (1971). A lower Lipschitz condition for the stable subordinator. $Z$. Wahrscheinlichkeitstheorie verw. Gebiete 17, 23-32.

[70] J. Hawkes (1975). On the potential theory of subordinators. Z. Wahrscheinlichkeitstheorie verw. Gebiete 33, 113-132.

[71] J. Hawkes (1977). Intersection of Markov random sets. Z. Wahrscheinlichkeitstheorie verw. Gebiete 37, 243-251.

[72] F. Hirsch (1984). Générateurs étendus et subordination au sens de Bochner In: Séminaire de Théorie du Potentiel 7, Lecture Notes in Maths. 1061 pp. 134-156. Springer, Berlin.

[73] D. G. Hobson (1994). Asymptotics for an arcsine type result. Ann. Inst. Henri Poincaré 30, 235-243.

[74] J. Hoffmann-Jørgensen (1969). Markov sets. Math. Scand. 24, 145-166.

[75] E. Hopf (1950). The partial differential equation $u_{t}+u u_{x}=\mu u_{x x}$. Comm. Pure Appl. Math. 3, 201-230.

[76] J. Horowitz (1968). The Hausdorff dimension of the sample path of a subordinator. Israel J. Math. 6, 176-182.

[77] J. Horowitz (1972). Semilinear Markov processes, subordinators and renewal theory. Z. Wahrscheinlichkeitstheorie verw. Gebiete 24, 167-193.

[78] X. Hu and S. J. Taylor (1997). The multifractal structure of stable occupation measure. Stochastic Process. Appl. 66, 283-299.

[79] Y. Hu and Z. Shi (1997). Extreme lengths in Brownian and Bessel excursions. Bernoulli 3, 387-402.

[80] B. Huff (1969). The strict subordination of a differential process. Sankhya Sera. A 31, 403-412. 
[81] K. Itô (1942). On stochastic processes. I. (Infinitely divisible laws of probability). Japan J. Math. 18, 261-301.

[82] K. Itô (1970). Poisson point processes attached to Markov processes. In: Proc. 6th Berkeley Symp. Math. Stat. Prob. III, 225-239.

[83] K. Itô and H. P. McKean (1965). Diffusion processes and their sample paths. Springer, Berlin.

[84] N. Jacob and R. L. Schilling (1996). Subordination in the sense of S. Bochner An approach through pseudo differential operators. Math. Nachr. 178, 199-231.

[85] N. Jacob and R. L. Schilling (1997). Some Dirichlet spaces obtained by subordinate reflected diffusions. Rev. Mat. Iberoamericana 15, 59-91.

[86] S. Jaffard: The multifractal nature of Lévy processes. Preprint.

[87] N. C. Jain and W. E. Pruitt (1987). Lower tail probabilities estimates for subordinators and nondecreasing random walks. Ann. Probab. 15, 75-102.

[88] M. Jeanblanc, J. Pitman and M. Yor (1997). The Feynman-Kac formula and decomposition of Brownian paths. Computat. Appl. Math. 6.1, 27-52.

[89] T. Jeulin and M. Yor (1981). Sur les distributions de certaines fonctionnelles du mouvement brownien. In: Séminaire de Probabilités XV, Lecture Notes in Math. 850, pp. 210-226. Springer, Berlin.

[90] J. P. Kahane (1985). Some random series of functions. 2nd edn. Cambridge University Press, Cambridge.

[91] J. P. Kahane (1990). Recouvrements aléatoires et théorie du potentiel. Colloquium Mathematicum LX/LXI, 387-411.

[92] Y. Kasahara (1975). Spectral theory of generalized second order differential operators and its applications to Markov processes. Japan J. Math. 1, 67-84.

[93] D. G. Kendall (1968). Delphic semigroups, infinitely divisible regenerative phenomena, and the arithmetic of $p$-functions. Z. Wahrscheinlichkeitstheorie verw. Gebiete 9, 163-195.

[94] J. T. Kent (1980). Eigenvalues expansions for diffusions hitting times. $Z$. Wahrscheinlichkeitstheorie verw. Gebiete 52, 309-319.

[95] J. T. Kent (1982). The spectral decomposition of a diffusion hitting time. Ann. Probab. 10, 207-219.

[96] H. Kesten (1969). Hitting probabilities of single points for processes with stationary independent increments. Memoirs Amer. Math. Soc. 93.

[97] H. Kesten (1970). The limit points of random walk. Ann. Math. Stat. 41, 1173-1205. 
[98] D. Khoshnevisan (1997). The rate of convergence in the ratio ergodic theorem for Markov processes. Preprint.

[99] D. Khoshnevisan and T. M. Lewis (1997). Stochastic calculus for Brownian motion on a Brownian fracture. Preprint.

[100] J. F. C. Kingman (1972). Regenerative phenomena. Wiley, London.

[101] F. B. Knight (1981). Characterization of the Lévy measure of inverse local times of gap diffusions. In: Seminar on Stochastic Processes 1981, pp. 53-78, Birkhäuser.

[102] S. Kotani and S. Watanabe (1981). Krein's spectral theory of strings and general diffusion processes. In: Functional Analysis in Markov Processes (ed M. Fukushima), Proceeding Katata and Kyoto 1981, Lecture Notes in Math. 923 pp. 235-259, Springer.

[103] U. Küchler (1986). On sojourn times, excursions and spectral measures connected with quasi diffusions. J. Math. Kyoto Univ. 26, 403-421.

[104] U. Küchler and P. Salminen (1989). On spectral measures of strings and excursions of quasi diffusions. In: Séminaire de Probabilités XXIII, Lecture Notes in Math. 1372 pp. 490-502, Springer.

[105] A. Lachal: Sur la distribution de certaines fonctionnelles de l'intégrale du mouvement brownien avec dérive parabolique et cubique. Comm. Pure Appl. Math. XLIX-12, 1299-1338.

[106] J. Lamperti (1962). An invariance principle in renewal theory. Ann. Math. Stat. 33, 685-696.

[107] J. F. Le Gall (1987). Mouvement brownien, cônes et processus stables. Probab. Theory Relat. Fields 76, 587-627.

[108] J. F. Le Gall (1992). Some properties of planar Brownian motion. In: Ecole d'été de Probabilités de St-Flour XX, Lecture Notes in Maths. 1527, pp. 111235. Springer, Berlin.

[109] B. Maisonneuve (1971). Ensembles régénératifs, temps locaux et subordinateurs. In: Séminaire de Probabilités V, Lecture Notes in Math. 191, pp. 147-169. Springer, Berlin

[110] B. Maisonneuve (1974). Systèmes régénératifs. Astérisque 15, Société Mathématique de France.

[111] B. Maisonneuve (1983). Ensembles régénératifs de la droite. Z. Wahrscheinlichkeitstheorie verw. Gebiete 63, 501-510.

[112] B. Maisonneuve (1993). Processus de Markov: Naissance, retournement, régénération. In: Ecole d'été de Probabilités de Saint-Flour XXI-1991. Lecture Notes in Maths. 1541, Springer. 
[113] B. B. Mandelbrot (1972). Renewal sets and random cutouts. Z. Wahrscheinlichkeitstheorie verw. Gebiete 22, 145-157.

[114] M. B. Marcus and J. Rosen (1992). Sample path properties of the local times of strongly symmetric Markov processes via Gaussian processes. Ann. Probab. 20, 1603-1684.

[115] M. B. Marcus and J. Rosen (1994). Laws of the iterated logarithm for the local times of symmetric Lévy processes and recurrent random walks. Ann. Probab. 22, 626-658.

[116] M. B. Marcus and J. Rosen (1994). Laws of the iterated logarithm for the local times of recurrent random walks on $Z^{2}$ and of Lévy processes and recurrent random walks in the domain of attraction of Cauchy random variables. Ann. Inst. Henri Poincaré 30, 467-499.

[117] L. Marsalle (1997). Applications des subordinateurs à l'étude de trois familles de temps exceptionnels. Thèse d'Université. Laboratoire de Probabilités de l'Université Pierre et Marie Curie.

[118] L. Marsalle (1999). Slow points and fast points of local times. Ann. Probab. 27, 150-165.

[119] P. A. Meyer (1984). Transformation de Riesz pour les lois gaussiennes. In: Séminaire de Probabilités XVIII, Lecture Notes in Maths. 1059 pp. 179-193. Springer, Berlin.

[120] I. S. Molchanov (1993). Intersection and shift functions of strong Markov random closed sets. Prob. Math. Stats. 14-2, 265-279.

[121] I. S. Molchanov and E. Ostrovski (1969). Symmetric stable processes as traces of degenerate diffusion processes. Th. Prob. Appl. 14, 128-131.

[122] J. Neveu (1961). Une généralisation des processus à accroissements positifs indépendants. Abh. Math. Sem. Univ. Hamburg 25, 36-61.

[123] S. Orey (1967). Polar sets for processes with stationary independent increments. In: Markov processes and potential theory, pp. 117-126. Wiley, New-York.

[124] S. Orey and S. J. Taylor (1974). How often on a Brownian path does the law of the iterated logarithm fail? Proc. London Math. Soc. 28, 174-192.

[125] J. W. Pitman (1986). Stationary excursions. Séminaire de Probabilités XXI, Lecture Notes in Maths. 1247 pp. 289-302. Springer, Berlin.

[126] J. W. Pitman and M. Yor (1992). Arc sine laws and interval partitions derived from a stable subordinator. Proc. London Math. Soc. 65, 326-356.

[127] J. W. Pitman and M. Yor (1997). The two-parameter Poisson-Dirichlet distribution derived from a stable subordinator. Ann. Probab. 25, 855-900. 
[128] J. W. Pitman and M. Yor (1997). On the lengths of excursions of some Markov processes. Séminaire de Probabilités XXXI, Lecture Notes in Maths. 1655 pp. 272-286. Springer, Berlin.

[129] J. W. Pitman and M. Yor (1997). On the relative lengths of excursions derived from a stable subordinator. Séminaire de Probabilités XXXI Lecture Notes in Maths. 1655 pp. 287-305. Springer, Berlin.

[130] W. E. Pruitt (1991). An integral test for subordinators. In: Random Walks, Brownian Motion and Iteracting Particle Systems: A Festschrift in honor of Frank Spitzer, pp. 389-398. Birkhäuser, Boston.

[131] B. Rajeev and M. Yor (1995). Local times and almost sure convergence of semimartingales. Ann. Inst. Henri Poincaré 31 653-667.

[132] D. Revuz and M. Yor (1994). Continuous martingales and Brownian motion, 2nd edn. Springer, Berlin.

[133] C. A. Rogers (1970). Hausdorff measure. Cambridge University Press, Cambridge.

[134] L. C. G. Rogers (1983). Wiener-Hopf factorization of diffusions and Lévy processes. Proc. London Math. Soc. 47, 177-191.

[135] L. C. G. Rogers (1989). Multiple points of Markov processes in a complete metric space. In: Séminaire de Probabilités XXIII. Lecture Note in Maths. 1372 pp. 186-197. Springer, Berlin.

[136] L. C. G. Rogers (1989). A guided tour through excursions. Bull. London Math. Soc. 21, 305-341.

[137] L. C. G. Rogers and D. Williams (1987). Diffusions, Markov processes, and martingales vol. 2: Itô calculus. Wiley, New-York.

[138] L. C. G. Rogers and D. Williams (1994). Diffusions, Markov processes, and martingales vol. I: Foundations. (First edition by D. Williams, 1979) Wiley, New-York.

[139] B. A. Rogozin (1966). On the distribution of functionals related to boundary problems for processes with independent increments. Th. Prob. Appl. 11, 580591.

[140] Ya. Sinai (1992). Statistics of shocks in solution of inviscid Burgers equation. Commun. Math. Phys. 148, 601-621.

[141] M. J. Sharpe (1989). General theory of Markov processes. Academic Press, New-York.

[142] Z. S. She, E. Aurell and U. Frisch (1992). The inviscid Burgers equation with initial data of Brownian type. Commun. Math. Phys. 148, 623-641.

[143] L. A. Shepp (1972). Covering the line with random intervals. Z. Wahrscheinlichkeitstheorie verw. Gebiete 23, 163-170. 
[144] A. N. Shiryaev (1984).Probability. Springer-Verlag, New York.

[145] F. Spitzer (1958). Some theorems concerning two-dimensional Brownian motion. Trans. Amer. Math. Soc. 87, 187-197.

[146] S. J. Taylor (1973). Sample path properties of processes with stationary independent increments. In: Stochastic Analysis, pp. 387-414. Wiley, London.

[147] S. J. Taylor (1986). The use of packing measure in the analysis of random sets. In: Stochastic Processes and their Applications (eds K. Itô and T. Hida), Proceedings Nagoya 1985, Lecture Notes in Maths 1203, pp. 214-222. Springer, Berlin.

[148] S. J. Taylor and C. Tricot (1985). Packing measure and its evaluation for a Brownian path. Trans. Amer. Math. Soc. 288, 679-699.

[149] M. Tomisaki (1977). On the asymptotic behaviors of transition probability densities of one-dimensional diffusion processes. Publ. RIMS Kyoto Univ. 12, 819837.

[150] S. Watanabe (1975). On time-inversion of one-dimensional diffusion processes. Z. Wahrscheinlichkeitstheorie verw. Gebiete 31, 115-124.

[151] S. Watanabe (1995). Generalized arcsine laws for one-dimensional diffusion processes and random walks. In: Stochastic Analysis (eds M. Cranston and M. Pinsky), Proceeding of Symposia in Pure Math. 57, pp. 157-172. American Mathematical Society.

[152] M. Yor (1995). Local times and excursions for Brownian motion: a concise introduction. Lecciones en Matemáticas 1. Facultad de Ciencias, Universidad Central de Venezuela.

[153] M. Yor (1997). Some aspects of Brownian motion. Part II: Some recent martingale problems. Birkhäuser, Basel. 